DOI $10.4171 /$ JEMS/313

Robert M. Guralnick · Pham Huu Tiep

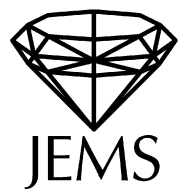

\title{
A problem of Kollár and Larsen on finite linear groups and crepant resolutions
}

Received June 2, 2009

\begin{abstract}
The notion of age of elements of complex linear groups was introduced by M. Reid and is of importance in algebraic geometry, in particular in the study of crepant resolutions and of quotients of Calabi-Yau varieties. In this paper, we solve a problem raised by J. Kollár and M. Larsen on the structure of finite irreducible linear groups generated by elements of age $\leq 1$. More generally, we bound the dimension of finite irreducible linear groups generated by elements of bounded deviation. As a consequence of our main results, we derive some properties of symmetric spaces $G U_{d}(\mathbb{C}) / G$ having shortest closed geodesics of bounded length, and of quotients $\mathbb{C}^{d} / G$ having a crepant resolution.
\end{abstract}

Keywords. Age, deviation, finite linear groups, complex reflection groups, crepant resolutions

\section{Introduction}

Let $V=\mathbb{C}^{d}$ be a $d$-dimensional complex space and let $G<G L(V)$ be a finite subgroup. A classical theme in group theory and representation theory, going back at least to work of H. Blichfeldt on primitive linear groups, and work of G. C. Shephard and J. A. Todd [ST] on complex reflection groups, is to characterize $G$ under various conditions that force $G$ to contain non-identity elements which are "close" to the identity transformation on $V$. Recall that a complex reflection group (c.r.g. for short) is a subgroup of $G U(V)$ that is generated by a set of complex (pseudo)reflections. The complex reflection groups can be arguably said to be one of the most ubiquitous objects in modern mathematics.

Recently, motivated by potential applications in algebraic geometry, string theory, mirror symmetry, and quantum cohomology, J. Kollár and M. Larsen [KL] have raised the problem of studying linear groups containing elements of bounded (or small) deviation, where the deviation is defined in a certain way to measure the "closeness" of group elements to the identity transformation. It turns out to be most convenient to work with the following $L^{2}$-variant of the Kollár-Larsen deviation: $d_{2}(g)^{2}=2(\operatorname{dim}(V)-|\operatorname{Tr}(g)|)$

R. M. Guralnick: Department of Mathematics, University of Southern California, Los Angeles, CA 90089-1113, USA; e-mail: guralnic@math.usc.edu

Pham Huu Tiep: Department of Mathematics, University of Arizona, Tucson, AZ 85721-0089, USA; e-mail: tiep@ math.arizona.edu

Mathematics Subject Classification (2010): 20C15, 14E15 
for $g \in G L(V)$ (see $\$ 2.2$, in particular, Corollary 2.12 and Proposition 2.17(iii), for various notions of deviation and their relationships). Henceforth we say that a subgroup $G \leq G L(V)$ has property $\mathcal{P}$ up to scalars if there is a subgroup $H \leq G L(V)$ with property $\mathcal{P}$ such that $Z(G L(V)) G=Z(G L(V)) H$.

The first main result of the paper is the following theorem which bounds the dimension of the representation in terms of the deviations of generators.

Theorem 1.1. Let $G<G L(V)$ be a finite irreducible subgroup. Assume that there is a constant $C \geq 4$ such that, up to scalars, $G$ is generated by some elements $g_{i}$ with $d_{2}\left(g_{i}\right)^{2} \leq C, 1 \leq i \leq s$. Then one of the following holds.

(i) $\operatorname{dim}(V) \leq \mathfrak{f}(C):=\max \left\{4 C^{2} / 63,40 C\right\}$.

(ii) $Z(G) \times \mathrm{A}_{n} \leq G \leq\left(Z(G) \times \mathrm{A}_{n}\right) \cdot 2$ and $\operatorname{dim}(V)=n-1$, with $\mathrm{A}_{n}$ acting on $V$ as on its deleted natural permutation module.

(iii) $G$ preserves a decomposition $V=V_{1} \oplus \cdots \oplus V_{m}$, with $\operatorname{dim}\left(V_{i}\right) \leq C / 4$ and $G$ inducing either $\mathrm{S}_{m}$ or $\mathrm{A}_{m}$ while permuting the $m$ subspaces $V_{1}, \ldots, V_{m}$.

One certainly expects the upper bound $\operatorname{dim}(V) \leq \mathfrak{f}(C)$ in Theorem 1.1(i) to have rather a theoretical than practical value. However, we notice that for $C$ large enough (say $C \geq 630$ ), this bound is already quite close to optimal (cf. Example 4.2). In general, as pointed out to the authors by Kollar, Theorem 1.1 should have interesting implications for differential geometry on symmetric spaces. Consider for instance locally symmetric spaces that behave locally like $G U_{n}(\mathbb{C})$ : they are of the form $G U_{n}(\mathbb{C}) / G$ for a finite subgroup $G<G U_{n}(\mathbb{C})$. Then the shortest closed geodesics in $G U_{n}(\mathbb{C}) / G$ have length $2 \pi \min _{1 \neq g \in G}\|g\|$, where $\|g\|$ is as defined in Definition 2.8. Here is one consequence of Theorem 1.1 in this context.

Corollary 1.2. Let $G<G U(V)$ be a finite irreducible, primitive, tensor indecomposable subgroup. Assume that the shortest closed geodesics in $G U(V) / G$ have length $\leq L$. Then either one of the conclusions (i), (ii) of Theorem 1.1 holds for $G$ with $C:=\max \left\{4, L^{2}\right\}$, or $\operatorname{dim}(V) \leq(L \cdot|Z(G)| / 2 \pi)^{2}$.

The next result shows that non-central elements $g$ of finite irreducible subgroups of $G L(V)$ usually have $d_{2}(g)^{2} \geq 4$, which implies that the condition $C \geq 4$ in Theorem 1.1 is natural.

Theorem 1.3. Let $G<G L(V)$ be a finite primitive, irreducible subgroup. Let $d:=$ $\operatorname{dim}(V) \geq 2, g \in G \backslash Z(G)$, and set $\Delta(g):=\operatorname{dim}(V)-|\operatorname{Tr}(g)|$. If $G$ is tensor induced, assume furthermore that $g$ acts non-trivially on the set of tensor factors of $V$. Then one of the following statements hold.

(i) $d=2$ and $\Delta(g) \geq(3-\sqrt{5}) / 2$.

(ii) $d=3$ and $\Delta(g) \geq 3-\sqrt{3}$.

(iii) $d=4$ and $\Delta(g) \geq 4-2 \sqrt{2}$.

(iv) $d \geq 5$ and either $\Delta(g) \geq 8-4 \sqrt{2}$, or $\Delta(g)=2$ and $g$ is a scalar multiple of $a$ reflection. 
(v) $V=A \otimes B$ is tensor decomposable as a G-module, $\operatorname{dim}(A)=2,2 \leq \operatorname{dim}(B) \leq 6$, $\left.g\right|_{B}$ is scalar, and $\Delta(g) \geq \operatorname{dim}(B) \cdot(3-\sqrt{5}) / 2$.

The notion of age of elements of complex linear groups (see Definition 2.1), originates from the work of M. Reid [R1], [R2], [IR]. Its importance in algebraic geometry comes from the Reid-Tai criterion [R1]: If the subgroup $G<G L_{d}(\mathbb{C})$ contains no complex reflections, then $\mathbb{C}^{d} / G$ is terminal, resp. canonical, if and only if age $(g)>1$, resp. age $(g) \geq 1$ for every $1 \neq g \in G$ (see e.g. [CK] for the definition of terminal and canonical singularities). This implies in particular the following result of [IR]: If $G$ is a finite subgroup of $G L_{d}(\mathbb{C})$ and $f: X \rightarrow \mathbb{C}^{d} / G$ is a crepant resolution, then $G$ contains elements $g$ with age $(g) \leq 1$. Recall that a resolution $f: X \rightarrow Y$ is said to be crepant if $f^{*} K_{Y}=K_{X}$. Furthermore, in the profound programme of S. Mori to classify 3-dimensional algebraic varieties, and in mirror symmetry, the singularities of type $\mathbb{C}^{d} / G$ for some finite subgroup $G<G L_{d}(\mathbb{C})$ form a very good test class where many features of the general case can be tested in a computable setting. Recently, there has been a tremendous amount of research devoted to crepant resolutions. For instance, minimal models in Mori's programme utilize crepant maps. Crepant resolutions of quotients $X / G$ of Calabi-Yau varieties $X$ are also used in works on mirror symmetry (particularly as a way of obtaining mirrors). Physicists have long believed that string theories on a quotient space and on its crepant resolutions should be equivalent. Recent conjectures of Y. Ruan [Ru], and J. Bryan and T. Graber [BG] state that if $f: X \rightarrow Y$ is a crepant resolution, then quantum cohomology of $X$ and of $Y$ are essentially the same. More recently, Kollár and Larsen [KL] studied quotients $X / G$ of a smooth projective Calabi-Yau variety $X$ by a finite group $G$ and showed in particular that the Kodaira dimension of $X / G$ is controlled by whether $\operatorname{Stab}_{x}(G)$ contains non-trivial elements of age $<1$ while acting on the tangent space $T_{x} X$ for some $x \in X$.

The next two theorems of the paper classify finite irreducible subgroups of $G L(V)$ that are generated by junior elements, that is, elements $g$ with $0<\operatorname{age}(g) \leq 1$, when $\operatorname{dim}(V)>8$.

Theorem 1.4. Let $V=\mathbb{C}^{d}$ with $d \geq 11$ and let $G<G L(V)$ be a finite irreducible subgroup. Assume that, up to scalars, $G$ is generated by its elements with age $\leq 1$. Then $G$ contains a complex bireflection of order 2 or 3 , and one of the following statements holds.

(i) $Z(G) \times \mathrm{A}_{d+1} \leq G \leq\left(Z(G) \times \mathrm{A}_{d+1}\right) \cdot 2$, with $\mathrm{A}_{d+1}$ acting on $V$ as on its deleted natural permutation module.

(ii) $G$ preserves a decomposition $V=V_{1} \oplus \cdots \oplus V_{d}$, with $\operatorname{dim}\left(V_{i}\right)=1$ and $G$ inducing either $\mathrm{S}_{d}$ or $\mathrm{A}_{d}$ while permuting the $d$ subspaces $V_{1}, \ldots, V_{d}$.

(iii) $2 \mid d$, and $G=D: \mathrm{S}_{d / 2}<G L_{2}(\mathbb{C})<\mathrm{S}_{d / 2}$, a split extension of $D<G L_{2}(\mathbb{C})^{d / 2}$ by $\mathrm{S}_{d / 2}$. Furthermore, if $g \in G \backslash D$ has age $(g) \leq 1$, then $g$ is a bireflection (and $\operatorname{age}(g)=1)$.

Theorem 1.5. Let $V=\mathbb{C}^{d}$ with $d \geq 9$ and let $G<G L(V)$ be a finite irreducible subgroup. Assume that, up to scalars, $G$ is generated by its elements with age $\leq 1$, and 
that $G$ contains a scalar multiple of a non-central element $g$ with age $(g)<1$. Then one of the following statements holds.

(i) One of the conclusions (i), (ii) of Theorem 1.4 holds, and $G$ contains a scalar multiple of a complex reflection.

(ii) The conclusion (iii) of Theorem 1.4 holds, and, modulo scalars, G cannot be generated by its elements of age $<1$.

The bound $d \geq 9$ in Theorem 1.5 is best possible (cf. Remark 5.15). In the case $4 \leq$ $\operatorname{dim}(V) \leq 10$ of Theorem 1.4, the structure of the arising subgroups $G$ is described in Proposition 5.16. On the contrary, from the group-theoretic viewpoint there is not much to say about the dimensions $\leq 3$ : if $1 \neq g \in S L_{d}(\mathbb{C})$ has finite order, then age $(g)=1$ if $d=2$, and either age $(g)$ or age $\left(g^{-1}\right)=1$ if $d=3$.

A key ingredient in the proofs of Theorems 1.4 and 1.5 comes from Proposition 2.17 and its consequence Corollary 2.18, which relate age $(g)$ to the $L^{2}$-deviation $d_{2}(g)^{2}$ and thus allow us to invoke available results on character ratios for finite quasi-simple groups [G], [GM]. Also, see Theorem 5.9 for a lower bound on the age of any non-central element in finite linear groups. One should compare the latter result with the classical theorem of Blichfeldt stating that the shortest arc of $S^{1}$ which contains all eigenvalues of a noncentral element in a finite primitive complex linear group has length at least $\pi / 3$.

In the case the finite subgroup $G<G L(V)$ fixes a non-degenerate symplectic form on $V$, D. Kaledin [Ka] and M. Verbitsky [V] have shown that $V / G$ can have a crepant resolution only when $G$ is generated by complex bireflections. In general, however, it is not true that (non-central) elements of age $\leq 1$ are always complex bireflections (nor elements with fixed point subspace of codimension 2). In this regard, one of the main assertions of Theorem 1.4 is the existence of complex bireflections in the groups $G$ satisfying the hypotheses of the theorem. If one knows that $G$ is generated by complex bireflections (or $G$ contains complex bireflections and is quasiprimitive), one can then appeal to available results on such groups, particularly [HW], [Hu], [Wa] (see also [Co]).

Interestingly, it was shown by V. Kac and K. Watanabe [KW], and independently by N. Gordeev [Gol], that if the $\operatorname{ring} \operatorname{Sym}(V)^{G}$ of $G$-invariants is a complete intersection for a finite group $G<G L(V)$, then $G$ is generated by elements with fixed point subspace of codimension 2. The finite groups $G<G L(V)$ with $\operatorname{Sym}(V)^{G}$ being a complete intersection have been classified by [Go2] and [N].

In a certain sense, Theorem 1.4 gives indications that crepant resolutions seem to occur mostly in low dimensions. Indeed, let $f: X \rightarrow \mathbb{C}^{d} / G$ be a crepant resolution, and let $K$ be the normal subgroup of $G$ generated by all elements of age $\leq 1$. Then by Theorem 1.4, for any irreducible summand $V$ of the $K$-module $\mathbb{C}^{d}$, either $\operatorname{dim}(V) \leq 10$, or the action of $K$ on $V$ contains complex bireflections (of order 2 or 3), and so the quotient $V / K$ should behave reasonably well from the point of view of algebraic geometry. (See [Ha] for the case of $S_{n}$ acting on the sum $\mathbb{C}^{n} \oplus \mathbb{C}^{n}$ of two copies of the natural permutation module.) We formulate one consequence of our results in this regard:

Corollary 1.6. Let $d \geq 11$ and let $G<G L_{d}(\mathbb{C})$ be a finite irreducible, primitive, tensor indecomposable subgroup. Assume that $\mathbb{C}^{d} / G$ is not terminal (for instance, it has a crepant resolution). Then one of the following statements holds. 
(i) $Z(G) \times \mathrm{A}_{d+1} \leq G \leq\left(Z(G) \times \mathrm{A}_{d+1}\right) \cdot 2$, with $\mathrm{A}_{d+1}$ acting on $\mathbb{C}^{d}$ as on its deleted natural permutation module.

(ii) All junior elements of $G$ are central, and $|Z(G)| \geq d$.

Recall that $(G, V)$ is a basic non-RT pair if $G<G L(V)$ is a finite irreducible subgroup and $G=\left\langle g^{G}\right\rangle$ for every non-central element $g \in G$ with age $(g)<1$. This notion was first introduced in [KL] and is of importance for the geometry of quotients of Calabi-Yau varieties. Our third main result is concerned with this notion and is in fact predicted by results of [KL].

Theorem 1.7. Let $G<G L(V)$ be a finite irreducible subgroup. Assume that, $G$ contains non-central elements $g \in G$ with age $(g)<1$, and that $G=\left\langle g^{G}\right\rangle$ for any such element. Assume in addition that $\operatorname{dim}(V)>4$. Then, up to scalars, $G$ is a complex reflection group.

Theorem 1.7 is not valid if $\operatorname{dim}(V)=4$. Examples of 4-dimensional basic non-RT pairs which are not projectively equivalent to a c.r.g. are given in [KL]; see also Examples 3.7 and 5.8. One should also compare Theorem 1.7 with the classical result that $\mathbb{C}^{d} / G$ is smooth if and only the finite subgroup $G<G L_{d}(\mathbb{C})$ is a complex reflection group (see e.g. [B, Theorem V.5.4]).

There should be similar results for representations in positive characteristic (where we consider the eigenvalues of semisimple elements), and similar algebro-geometric applications. There are results which indicate that if $G<G L(V)$ with $V$ finite-dimensional over an algebraically closed field, then $k[V]^{G}$ being a polynomial ring, resp. a complete intersection, implies that $G$ is generated by elements trivial on a subspace of codimension 1, resp. on a subspace of codimension at most 2 (cf. for instance [KM], [KW], [S]). Such groups have been classified (see [GS] for the last statement and references-also in [GS] finite and algebraic groups generated by symplectic reflections in all characteristics were classified). The authors have recently obtained some results on the values of Brauer characters which should be relevant.

\section{Preliminaries}

Let $V=\mathbb{C}^{n}$ be endowed with standard Hermitian form $(\cdot, \cdot)$; write $\|v\|=\sqrt{(v, v)}$ for any $v \in V$. Also let $S^{1}:=\{\lambda \in \mathbb{C}|| \lambda \mid=1\}$ and let $\mathcal{B}(V)$ be the collection of all orthonormal bases of $V$.

\subsection{Age}

Definition 2.1 ([IR], [R2]). Let $g \in G L(V)$ be conjugate to $\operatorname{diag}\left(e^{2 \pi i r_{1}}, \ldots, e^{2 \pi i r_{n}}\right)$, where $0 \leq r_{j}<1$. Then age $(g):=\sum_{j=1}^{n} r_{j}$.

Classical examples of non-scalar elements with age $<1$ are: reflections, complex reflections (or pseudoreflections), bireflections, and complex bireflections. These cases correspond to $\left(r_{1}, \ldots, r_{n}\right)=(1 / 2,0, \ldots, 0),\left(0<r_{1}<1,0, \ldots, 0\right),(1 / 2,1 / 2,0, \ldots, 0)$, and $\left(0<r_{1}<1,1-r_{1}, 0, \ldots, 0\right)$, respectively. (Note that all complex bireflections considered in this paper have determinant 1.) 
To deal with scalar multiples of linear tranformations, it is also convenient to define

$$
\operatorname{age}^{*}(g)=\inf _{\lambda \in S^{1}} \operatorname{age}(\lambda g)
$$

for any (diagonalizable) $g \in G U(V)$.

First we record the following observations, which we usually apply to linear transformations of finite order (as elements of $G L(V)$ ).

Lemma 2.2. The following statements hold for any $g \in G U(V)$.

(i) age $(g)$ and age* $(g)$ are well-defined, and constant on the $G U(V)$-conjugacy class of $g$.

(ii) There is some $\mu \in S^{1}$ (of finite order, if $|g|$ is finite) such that age* $(g)=\operatorname{age}(\mu g)$. In particular, $g$ is scalar if and only if age* $(g)=0$.

(iii) If $U \subseteq V$ is a g-invariant subspace then

$$
\operatorname{age}\left(\left.g\right|_{U}\right) \leq \operatorname{age}(g)=\operatorname{age}\left(\left.g\right|_{U}\right)+\operatorname{age}\left(\left.g\right|_{V / U}\right) .
$$

(iv) If $h \in G U(W)$, then

$$
\operatorname{age}^{*}(\operatorname{diag}(g, h)) \geq \operatorname{age}^{*}(g)+\operatorname{age}^{*}(h), \quad \operatorname{age}^{*}(g \otimes h) \geq \operatorname{dim}(W) \cdot \operatorname{age}^{*}(g) .
$$

(v) If $h \in G U(V)$ and $g h=h g$, then

$$
\operatorname{age}(g h) \leq \operatorname{age}(g)+\operatorname{age}(h), \quad \operatorname{age}^{*}(g h) \leq \operatorname{age}^{*}(g)+\operatorname{age}^{*}(h) .
$$

Proof. (i) and (iii) are obvious.

(ii) Let $e^{2 \pi i r_{1}}, \ldots, e^{2 \pi i r_{m}}$, where $0 \leq r_{1}<\cdots<r_{m}<1$, be the distinct eigenvalues of $g$. Consider the function $f(t):=\operatorname{age}\left(e^{-2 \pi i t} \cdot g\right)$ on the interval $(0,1]$. Note that $f$ is decreasing on each of the intervals $\left(0, r_{1}\right],\left(r_{1}, r_{2}\right], \ldots,\left(r_{m-1}, r_{m}\right],\left(r_{m}, 1\right]$. It follows that age* $(g)=\inf _{t \in(0,1]} f(t)$ is attained as the value of $f$ at one of the points $t=$ $r_{1}, r_{2}, \ldots, r_{m}, 1$. Thus we can take $\mu^{-1}$ to be either 1 or one of the eigenvalues of $g$, and so it has finite order in $S^{1}$ if $|g|$ is finite. (Also notice that if $m \geq 2$, then age* $(g) \geq$ $\min \left\{r_{2}-r_{1}, 1-\left(r_{2}-r_{1}\right)\right\}$.)

(iv) Without loss we may assume that $h=\operatorname{diag}\left(s_{1}, \ldots, s_{m}\right)$ with $s_{j} \in S^{1}$, and consider any $\lambda \in S^{1}$. Then by (iii) we have

$$
\begin{gathered}
\operatorname{age}(\lambda \cdot \operatorname{diag}(g, h))=\operatorname{age}(\lambda g)+\operatorname{age}(\lambda h) \geq \operatorname{age}^{*}(g)+\operatorname{age}^{*}(h), \\
\operatorname{age}(\lambda g \otimes h)=\operatorname{age}\left(\operatorname{diag}\left(\lambda s_{1} g, \ldots, \lambda s_{m} g\right)\right)=\sum_{j=1}^{m} \operatorname{age}\left(\lambda s_{j} g\right) \geq m \cdot \operatorname{age} e^{*}(g) .
\end{gathered}
$$

(v) Without loss we may assume that

$$
g=\operatorname{diag}\left(e^{2 \pi i r_{1}}, \ldots, e^{2 \pi i r_{m}}\right), \quad h=\operatorname{diag}\left(e^{2 \pi i s_{1}}, \ldots, e^{2 \pi i s_{m}}\right),
$$

with $0 \leq r_{j}, s_{j}<1$. Then $\operatorname{age}(g h) \leq \sum_{j=1}^{m}\left(r_{j}+s_{j}\right)=\operatorname{age}(g)+\operatorname{age}(h)$. Next, by (ii) there are $\alpha, \beta \in S^{1}$ such that age* $(g)=\operatorname{age}(\alpha g)$ and age* $(h)=\operatorname{age}(\beta h)$. Now

$\operatorname{age}^{*}(g h) \leq \operatorname{age}(\alpha \beta g h)=\operatorname{age}(\alpha g \cdot \beta h) \leq \operatorname{age}(\alpha g)+\operatorname{age}(\beta h)=\operatorname{age}^{*}(g)+\operatorname{age}^{*}(h)$. 
In fact, by the Chen-Ruan inequality [CR], Lemma 2.2(v) also holds without the condition $g h=h g$. Even more, the following inequality holds, where $V^{X}$ denotes the common fixed point subspace for any subset $X \leq G L(V)$.

Theorem 2.3 ([CR]). (i) If $x, y \in G U(V)$, then

$$
\operatorname{age}(x)+\operatorname{age}(y)-\operatorname{age}(x y)+\operatorname{dim}\left(V^{x, y}\right)-\operatorname{dim}\left(V^{x y}\right) \geq 0 .
$$

(ii) If $x, y, z \in G U(V)$ and $x y z=1$, then

$$
\operatorname{age}(x)+\operatorname{age}(y)+\operatorname{age}(z) \geq \operatorname{dim}(V)-\operatorname{dim}\left(V^{x, y, z}\right) .
$$

This theorem follows from the existence of a cohomology theory developed in [CR] (see also [Hep]). We will give an elementary proof of this result.

First we set up some notation. For $V=\mathbb{C}^{n}$ and $g \in G U(V)$, write $[g]=\left(v_{1}, \ldots, v_{n}\right)$ where $0 \leq v_{1} \leq \cdots \leq v_{n}<1$ and the eigenvalues of $g$ are $e^{2 \pi i v_{j}}, 1 \leq j \leq n$.

Lemma 2.4. Let $\operatorname{dim}(V)>1$ and $x, y \in G U(V)$, where $x$ is a complex reflection with $[x]=(r, 0, \ldots, 0), 0<r<1,[y]=\left(a_{1}, \ldots, a_{n}\right)$ and $[x y]=\left(b_{1}, \ldots, b_{n}\right)$. Let $H=\langle x, y\rangle$. Then the following conditions are equivalent:

(i) $H$ acts irreducibly.

(ii) $x$ and $y$ have no common eigenvector.

(iii) The collection $\left\{a_{1}, \ldots, a_{n}, b_{1}, \ldots, b_{n}\right\}$ consists of $2 n$ distinct elements.

Proof. If $H$ acts reducibly, then $x y=y$ on some non-trivial $H$-invariant space, whence $a_{j}=b_{k}$ for some $j, k$. Thus (iii) implies (i), and certainly (i) implies (ii).

Now assume (ii); in particular, neither $y$ nor $x y$ has an eigenvector on $V^{x}=u^{\perp}$ (for some $0 \neq u \in V$ ). Note that $x u=e^{2 \pi i r} u$. If $a_{i}=a_{j}$ for $i<j$, then $y$ has a two-dimensional eigenspace which therefore intersects $V^{x}$ non-trivially, a contradiction. Similarly, we see that $b_{i} \neq b_{j}$. Suppose now that both $x y$ and $y$ have a common eigenvalue $\beta$. In this case, again by (ii) we can find $v, w \in u^{\perp}$ such that $y(u+v)=\beta(u+v)$ and $x y(u+w)=\beta(u+w)$; in particular, $y(u+w)=e^{-2 \pi i r} \beta u+\beta w$. Thus $y(v-w)=$ $\beta\left(1-e^{-2 \pi i r}\right) u+\beta(v-w)$. Note that $|\beta|=1$ and $\|y(v-w)\|=\|v-w\|$ as $y \in G U(V)$. It follows that $e^{2 \pi i r}=1$, a contradiction.

The key to Theorem 2.3 is the following beautiful result [BH, Cor. 4.7] on eigenvalue interlacing (see also [MOW]).

Lemma $2.5([\mathrm{BH}])$. Let $x, y \in G U(V)$, where $x$ is a complex reflection with $[x]=$ $(r, 0, \ldots, 0), 0<r<1,[y]=\left(a_{1}, \ldots, a_{n}\right)$ and $[x y]=\left(b_{1}, \ldots, b_{n}\right)$. Assume that $a_{j}<a_{j+1}$ and $b_{j}<b_{j+1}$ for $1 \leq j<n$. Assume also that $a_{j} \neq b_{k}$ for any $j, k$. Then one of the following holds:

(a) $a_{1}<b_{1}<\cdots<a_{n}<b_{n}$; or

(b) $b_{1}<a_{1}<\cdots<b_{n}<a_{n}$.

Note that in either case $|\operatorname{age}(x y)-\operatorname{age}(y)|<1$, and so by considering determinants, if (a) above holds, then age $(x)+\operatorname{age}(y)=\operatorname{age}(x y)$, while if (b) holds, then age $(x)+\operatorname{age}(y)=$ $\operatorname{age}(x y)+1$. In any case, $\operatorname{age}(x)+\operatorname{age}(y) \geq \operatorname{age}(x y)$. 
We now sketch an elementary proof of Lemma 2.5.

Proof. 1) Clearly, we may assume $\operatorname{dim}(V)>1$. Let $V^{x}=u^{\perp}$ for some $0 \neq u \in V$. By Lemma 2.4, y cannot have any eigenvector in $u^{\perp}$ or $\langle u\rangle_{\mathbb{C}}$. For $t \in \mathbb{R} \backslash \mathbb{Z}$, let $x(t)$ be the complex reflection with $u^{\perp}$ as its reflecting hyperplane and $x(t) u=e^{2 \pi i t} u$. Also set $x(t)=1_{V}$ if $t \in \mathbb{Z}$.

Now let $w(t)=x(t) y$ for $t \in \mathbb{R}$. Note that by construction, for any $t, t^{\prime} \in \mathbb{R}$ with $t-t^{\prime} \notin \mathbb{Z}, w(t)$ and $w\left(t^{\prime}\right)$ cannot have any common eigenvector. (Otherwise $x\left(t-t^{\prime}\right.$ ) and $y$ have a common eigenvector $v$. This $v$ must be either in $u^{\perp}$ or $\langle u\rangle_{\mathbb{C}}$, contrary to the aforementioned property of $y$.) It then follows by Lemma 2.4 that, when $0 \leq t<t^{\prime}<1$, all the $n$ eigenvalues of $w(t)$ are distinct, and $w(t)$ and $w\left(t^{\prime}\right)$ have no common eigenvalue.

2) Define $a_{k n+i}=a_{i}+k$ for $k \in \mathbb{Z}$. Also, let $[w(r)]=\left(b_{1}(r), \ldots, b_{n}(r)\right)$ and consider any $1 \leq j \leq n$. By the conclusion of 1$), a_{i}<b_{j}(r)<a_{i+1}$ for some $i \in \mathbb{Z}$. Note that the spectrum of $w(t)$ depends continuously on $t \in \mathbb{R}$. Hence, for $t$ in some small neighborhood of $r$, the $j^{\text {th }}$ entry $b_{j}(t)$ of $[w(t)]$ satisfies $a_{i}<b_{j}(t)<a_{i+1}$. Let

$$
X:=\left\{s \mid r \leq s<1, a_{i}<b_{j}(t)<a_{i+1} \text { for all } t \in[r, s]\right\} .
$$

We claim that $X=[r, 1)$. Indeed, let $f:=\sup X \leq 1$ and assume $f<1$. Then there is a sequence $\left\{s_{n}\right\} \subseteq X$ such that $\lim _{n \rightarrow \infty} s_{n}=f$. The spectrum continuity implies that $a_{i} \leq b_{j}(f) \leq a_{i+1}$. Since $0<f<1$, we must have $a_{i}<b_{j}(f)<a_{i+1}$. It is now easy to check that there is some $\epsilon>0$ such that $f+\epsilon \in X$, a contradiction. Thus $f=1$, which in turn implies that $X=[r, 1)$. Similarly,

$$
\left\{s \mid 0 \leq s<r, \forall t \in[s, r], a_{i}<b_{j}(t)<a_{i+1}\right\}=(0, r] .
$$

We have shown that

$$
a_{i}<b_{j}(t)<a_{i+1} \quad \text { for all } t \in(0,1) .
$$

3) Replacing $(x, y)$ by $\left(x^{-1}, x y\right)$ if necessary, we may assume that $a_{1}<b_{1}$. Note that $b_{1}(0)=a_{1}$. Hence $b_{1}(t)$ is close to $a_{1}$ when $t \in(0,1)$ is small enough and so (1) implies that $a_{1}<b_{1}(t)<a_{2}$ for all $t \in(0,1)$; in particular, $b_{1}=b_{1}(r)<a_{2}$. Also, $a_{1} \leq b_{1}(1)=\lim _{t \rightarrow 1} b_{1}(t) \leq a_{2}$. Since $b_{1}(1)$ is some $a_{k}$, we get $b_{1}(1) \in\left\{a_{1}, a_{2}\right\}$. Moreover, if $b_{1}(1)=a_{1}=b_{1}(0)$, then the continuity of $b_{1}(t)$ on $[0,1]$ implies that some $w(t), w\left(t^{\prime}\right)$ with $0<t<t^{\prime}<1$ have a common eigenvalue, contrary to the conclusion of 1$)$. So $b_{1}(1)=a_{2}$.

4) Next, $b_{2}(0)=a_{2}$. If $b_{2}=b_{2}(r)>a_{2}$, then, as above, (1) implies that $a_{2}<$ $b_{2}(t)<a_{3}$ for all $t \in(0,1)$. Assume the contrary: $b_{2}<a_{2}$. Again by (1) we must now have $a_{1}<b_{2}(t)<a_{2}$ for all $t \in(0,1)$. Arguing as in 3) we get $b_{2}(1) \in\left\{a_{1}, a_{2}\right\}$ and $b_{2}(1) \neq b_{2}(0)=a_{2}$, i.e. $b_{2}(1)=a_{1}$. On the other hand, $b_{2}(t) \geq b_{1}(t)$ for all $t \in[0,1]$, whence $b_{2}(1) \geq b_{1}(1)=a_{2}$, a contradiction. We have shown that $a_{2}<b_{2}(t)<a_{3}$ for all $t \in(0,1)$. Continuing in the same fashion, we get $a_{j}<b_{j}(t)<a_{j+1}$ for all $j$ and $t \in(0,1)$.

Proof of Theorem 2.3. Notice that age $(z)=\operatorname{dim}(V)-\operatorname{age}(x y)-\operatorname{dim}\left(V^{x y}\right)$, so (i) and (ii) are equivalent. Next, diagonalize $x$ and then write $x$ as a product of $m \leq n$ commuting 
complex reflections. To prove statement (i), we proceed by induction on $m$. First assume that $x$ is a complex reflection. Let $H=\langle x, y\rangle$. If $H$ does not act irreducibly, the result follows by induction on $\operatorname{dim}(V)$ (by writing $V=W \perp W^{\perp}$ where $W$ is $H$-invariant). So assume this is not the case; in particular, $V^{x, y}=0$. We need to prove that: $\operatorname{age}(x)+$ $\operatorname{age}(y) \geq \operatorname{age}(x y)+\operatorname{dim}\left(V^{x y}\right)$. By Lemma 2.4, no eigenspace of $y$ or $x y$ has dimension more than 1, and $x y$ and $y$ have no common eigenvalues. By Lemma 2.5 and the remarks before its proof, age $(x)+\operatorname{age}(y) \geq \operatorname{age}(x y)$. If $x y$ has no trivial eigenvalue we are done. So we may assume that $x y$ does have exactly one trivial eigenvalue, whence $y$ has no trivial eigenvalue. Thus the case (b) of Lemma 2.5 holds and so age $(x)+\operatorname{age}(y)=$ age $(x y)+1$ as desired.

For the induction step, write $x=s x^{\prime}$ where $s$ is a complex reflection and $x^{\prime}$ is a product of $m-1$ complex reflections and age $(x)=\operatorname{age}(s)+\operatorname{age}\left(x^{\prime}\right)$. By the complex reflection case,

$$
\operatorname{age}(s)+\operatorname{age}\left(x^{\prime} y\right)+\operatorname{dim}\left(V^{s, x^{\prime} y}\right) \geq \operatorname{age}(x y)+\operatorname{dim}\left(V^{x y}\right) .
$$

By induction,

$$
\operatorname{age}\left(x^{\prime}\right)+\operatorname{age}(y)+\operatorname{dim}\left(V^{x^{\prime}, y}\right) \geq \operatorname{age}\left(x^{\prime} y\right)+\operatorname{dim}\left(V^{x^{\prime} y}\right) .
$$

Note that $V^{s, x^{\prime} y} \cap V^{x^{\prime}, y}=V^{s, x^{\prime}, y} \subseteq V^{x, y}$ and $V^{s, x^{\prime} y}, V^{x^{\prime}, y} \subseteq V^{x^{\prime} y}$, whence

$$
\operatorname{dim}\left(V^{x^{\prime} y}\right)+\operatorname{dim}\left(V^{x, y}\right) \geq \operatorname{dim}\left(V^{s, x^{\prime} y}\right)+\operatorname{dim}\left(V^{x^{\prime}, y}\right) .
$$

The last three relations on dimensions readily imply

$\operatorname{age}(x)+\operatorname{age}(y)=\operatorname{age}(s)+\operatorname{age}\left(x^{\prime}\right)+\operatorname{age}(y) \geq \operatorname{age}(x y)+\operatorname{dim}\left(V^{x y}\right)-\operatorname{dim}\left(V^{x, y}\right)$.

\subsection{The set-up $(\star)$}

We are interested in finite subgroups of $G L(V)$ that contain non-trivial elements of age $<1$, resp. $\leq 1$. Of course it would be very difficult to classify these groups without extra assumptions on them.

Lemma 2.6. Let $W$ be a finite-dimensional vector space over $\mathbb{C}$ and let $G<G L(W)$ be a finite subgroup containing a non-trivial element $g$ with age $(g)<1$, resp. age $(g) \leq 1$. Then there is a normal subgroup $K \triangleleft G$ and a nonzero $K$-invariant subspace $V$ of $W$ such that all the following conditions hold:

(i) $K$ acts irreducibly on $V$;

(ii) $g \in K$ and $0<\operatorname{age}\left(\left.g\right|_{V}\right)<1$, resp. $0<\operatorname{age}\left(\left.g\right|_{V}\right) \leq 1$;

(iii) $K$ is generated by the set of its elements whose restrictions to $V$ have age $<1$, resp. $\leq 1$.

In fact, if $0 \neq U \subseteq W$ is any $K$-submodule, then $K$ is generated by the set of its elements whose restrictions to $U$ have age $<1$, resp. $\leq 1$. 
Proof. Let $\mathcal{X}$ denote the set of all non-trivial elements of $G$ that have age $<1$, resp. $\leq 1$, and define $K=\langle\mathcal{X}\rangle$. Then $K \triangleleft G$ and $K \ni g$. Decompose $W$ into a direct sum $\bigoplus_{i=1}^{s} V_{i}$ of irreducible $K$-submodules. We may assume by Lemma 2.2 that $0<\operatorname{age}\left(\left.g\right|_{V_{1}}\right)<1$, resp. $0<\operatorname{age}\left(\left.g\right|_{V_{1}}\right) \leq 1$. Let $\mathcal{Y}:=\left\{h \in K \mid\right.$ age $\left(\left.h\right|_{V_{1}}\right)<1$, resp. $\left.\leq 1\right\}$. Observe that $\mathcal{X} \subseteq \mathcal{Y}$, whence $K=\langle\mathcal{Y}\rangle$. Thus $V:=V_{1}$ satisfies (i)-(iii).

Next, let $0 \neq U \subseteq W$ be any $K$-submodule and let $\mathcal{Y}^{\prime}:=\left\{h \in K \mid \operatorname{age}\left(\left.h\right|_{U}\right)<1\right.$, resp. $\leq 1\}$. Then again $\mathcal{X} \subseteq \mathcal{Y}^{\prime}$ and so $K=\left\langle\mathcal{Y}^{\prime}\right\rangle$.

Lemma 2.6 shows that it is natural to restrict our attention to the following set-up, which is slightly more general than the one considered in $[\mathrm{KL}]$ :

$(\star) \quad G$ is a finite irreducible subgroup of $\mathcal{G}=G L(V)$ and $Z(\mathcal{G}) G=\langle\mathcal{X}\rangle$, where $\mathcal{X}:=$ $\{g \in Z(\mathcal{G}) G \mid 0<\operatorname{age}(g)<1$, resp. $0<\operatorname{age}(g) \leq 1\}$.

The condition $(\star)$ means that, up to scalars, the finite irreducible subgroup $G<G L(V)$ is generated by some non-trivial elements with age $<1$, resp. $\leq 1$. In fact we can even assume that these generators are non-scalar:

Remark 2.7. Assume $G$ satisfies $(\star)$ and $\operatorname{dim}(V)>1$. Then $\mathcal{X}^{*} \neq \emptyset$ and $Z(\mathcal{G}) G=$ $Z(\mathcal{G})\left\langle\mathcal{X}^{*}\right\rangle$, where $\mathcal{X}^{*}:=\left\{g \in G \mid 0<\operatorname{age}^{*}(g)<1\right.$, resp. $0<$ age* $\left.^{*}(g) \leq 1\right\}$. Indeed, if $\mathcal{X}^{*}=\emptyset$ then all $h \in \mathcal{X}$ are scalar and so is $G$, contradicting the condition $\operatorname{dim}(V)>1$. Next, any $x \in G$ can be written as $g_{1} \ldots g_{m}$ with $g_{i}=\alpha_{i} h_{i} \in \mathcal{X}, \alpha_{i} \in S^{1}, h_{i} \in G$, and $h_{i} \in \mathcal{X}^{*}$ precisely when $i \in J$ for some subset $J \subseteq\{1, \ldots, m\}$. Then $x=\lambda \prod_{i \in J} h_{i} \in$ $Z(\mathcal{G})\left\langle\mathcal{X}^{*}\right\rangle$ for $\lambda=\prod_{i=1}^{m} \alpha_{i} \cdot \prod_{j \notin J} h_{j}$.

\subsection{Deviations}

A natural invariant metric on $G U(V)$ is defined as follows:

Definition 2.8. Let $T \in G L(V)$ be conjugate to $\operatorname{diag}\left(e^{2 \pi i r_{1}}, \ldots, e^{2 \pi i r_{n}}\right)$, where $0 \leq r_{j}$ $<1$. Then $\|T\|:=\left(\sum_{j=1}^{n} \min \left\{r_{j}, 1-r_{j}\right\}^{2}\right)^{1 / 2}$.

For our purposes it is more convenient to work with the following:

Definition 2.9. Let $j$ be any positive number and let $T \in G U(V)$ be any unitary linear operator. Then

$$
d_{j}(T):=\inf _{\lambda \in S^{1}, B \in \mathcal{B}(V)}\left(\sum_{b \in B}\|T(b)-\lambda b\|^{j}\right)^{1 / j} .
$$

This definition is a slight generalization of [KL, Definition 27] (where one takes $\lambda=1$ instead of the infimum over all $\lambda \in S^{1}$ ). First we list some basic properties of $d_{j}(T)$.

Lemma 2.10. Let $A, T \in G U(V)$ and $\alpha \in S^{1}$. Then the following hold:

(i) $d_{j}(T)=d_{j}(\alpha T)$.

(ii) $d_{j}(T)=d_{j}\left(A T A^{-1}\right)$.

(iii) $d_{j}(T)=d_{j}\left(T^{-1}\right)$. 
Proof. (i) Clearly $\alpha T \in G U(V)$. Consider any $\lambda \in S^{1}$ and $B \in \mathcal{B}(V)$. Then

$$
\sum_{b \in B}\|\alpha T(b)-\lambda b\|^{j}=\sum_{b \in B}\left\|T(b)-\alpha^{-1} \lambda b\right\|^{j} \geq d_{j}(T)^{j} .
$$

Taking the infimum over all $\lambda \in S^{1}$ and $B \in \mathcal{B}(V)$ we get $d_{j}(\alpha T) \geq d_{j}(T)$. Applying this inequality to $S:=\alpha T$ and $\alpha^{-1}$ we obtain $d_{j}(T)=d_{j}\left(\alpha^{-1} S\right) \geq d_{j}(S)=d_{j}(\alpha T)$, and the claim follows.

(ii) Consider any $\lambda \in S^{1}$ and $B \in \mathcal{B}(V)$. Then $A^{-1}(B) \in \mathcal{B}(V)$, and

$$
\begin{aligned}
\sum_{b \in B}\left\|A T A^{-1}(b)-\lambda b\right\|^{j} & =\sum_{c=A^{-1} b \in A^{-1}(B)}\|A(T(c)-\lambda c)\|^{j} \\
& =\sum_{c \in A^{-1}(B)}\|T(c)-\lambda c\|^{j} \geq d_{j}(T)^{j} .
\end{aligned}
$$

Taking the infimum over all $\lambda \in S^{1}$ and $B \in \mathcal{B}(V)$ we get $d_{j}\left(A T A^{-1}\right) \geq d_{j}(T)$. Applying this inequality to $S:=A T A^{-1}$ and $A^{-1}$ we obtain $d_{j}(T)=d_{j}\left(A^{-1} S\left(A^{-1}\right)^{-1}\right) \geq$ $d_{j}(S)=d_{j}\left(A T A^{-1}\right)$, and the claim follows.

(iii) Consider any $\lambda \in S^{1}$ and $B \in \mathcal{B}(V)$. Then

$$
\sum_{b \in B}\left\|T^{-1}(b)-\lambda b\right\|^{j}=\sum_{b \in B}\left\|\lambda^{-1} T\left(T^{-1}(b)-\lambda b\right)\right\|^{j}=\sum_{b \in B}\left\|T(b)-\lambda^{-1} b\right\|^{j} \geq d_{j}(T)^{j} .
$$

Taking the infimum over all $\lambda \in S^{1}$ and $B \in \mathcal{B}(V)$ we get $d_{j}\left(T^{-1}\right) \geq d_{j}(T)$. Applying this inequality to $S:=T^{-1}$ we get $d_{j}(T)=d_{j}\left(S^{-1}\right) \geq d_{j}(S)=d_{j}\left(T^{-1}\right)$, and so the claim follows.

Most of the time we will work with $d_{j}(T)$ where $j=1$ or 2 .

Lemma 2.11. For $T \in G U(V)$ the following hold:

(i) $d_{2}(T) \leq d_{1}(T) \leq \sqrt{\operatorname{dim}(V)} \cdot d_{2}(T)$.

(ii) $d_{1}(T) \geq \operatorname{dim}(V)-|\operatorname{Tr}(T)|$.

(iii) $d_{2}(T)^{2}=2(\operatorname{dim}(V)-|\operatorname{Tr}(T)|)$. Moreover, for any $B \in \mathcal{B}(V)$ we have

$$
d_{2}(T)=\inf _{\lambda \in S^{1}}\left(\sum_{b \in B}\|T(b)-\lambda b\|^{2}\right)^{1 / 2} .
$$

Proof. (i) For any $\lambda \in S^{1}$ and $B \in \mathcal{B}(V)$ we have

$$
d_{2}(T) \leq\left(\sum_{b \in B}\|T(b)-\lambda b\|^{2}\right)^{1 / 2} \leq \sum_{b \in B}\|T(b)-\lambda b\| .
$$

Taking the infimum over all $\lambda, B$ we get $d_{2}(T) \leq d_{1}(T)$. Next, again for any $\lambda \in S^{1}$ and $B \in \mathcal{B}(V)$ by the Cauchy-Schwarz inequality we have

$$
d_{1}(T) \leq \sum_{b \in B}\|T(b)-\lambda b\| \leq \sqrt{\operatorname{dim}(V)} \cdot\left(\sum_{b \in B}\|T(b)-\lambda b\|^{2}\right)^{1 / 2} .
$$

Taking the infimum over all $\lambda, B$ we get $d_{1}(T) \leq \sqrt{\operatorname{dim}(V)} \cdot d_{2}(T)$. 
(ii) Consider any $\lambda \in S^{1}$ and $B \in \mathcal{B}(V)$. Let $\left(a_{i j}\right)_{1 \leq i, j \leq n}$ be the matrix of $T$ in the basis $B$. Observe that

$$
\begin{aligned}
\sum_{b \in B}\|T(b)-\lambda b\| & =\sum_{l=1}^{n}\left(\sum_{k=1}^{n}\left|a_{k l}-\lambda \delta_{k, l}\right|^{2}\right)^{1 / 2} \geq \sum_{l=1}^{n}\left|\lambda^{-1} a_{l l}-1\right| \geq \sum_{l=1}^{n} \Re\left(1-\lambda^{-1} a_{l l}\right) \\
& =n-\Re\left(\lambda^{-1} \sum_{l=1}^{n} a_{l l}\right) \geq n-\left|\lambda^{-1} \sum_{l=1}^{n} a_{l l}\right|=\operatorname{dim}(V)-|\operatorname{Tr}(T)| .
\end{aligned}
$$

Taking the infimum over all $\lambda, B$ we arrive at the claim.

(iii) Consider an arbitrary $B \in \mathcal{B}(V)$ and let $A:=\left(a_{i j}\right)_{1 \leq i, j \leq n}$ be the matrix of $T$ in the basis $B$. For any $\lambda \in S^{1}$ we have

$$
\sum_{b \in B}\|T(b)-\lambda b\|^{2}=\sum_{l=1}^{n} \sum_{k=1}^{n}\left|\delta_{k, l}-\lambda^{-1} a_{k l}\right|^{2}=\sum_{1 \leq k, l \leq n} X_{k l} \bar{X}_{k l}=\operatorname{Tr}\left({ }^{t} \bar{X} \cdot X\right),
$$

where $X:=\left(\delta_{k, l}-\lambda^{-1} a_{k l}\right)_{1 \leq k, l \leq n}=I_{n}-\lambda^{-1} A$. Since $T \in G U(V)$, there is a matrix $C$ with ${ }^{t} \bar{C} \cdot C=I_{n}$ and a diagonal matrix $E=\operatorname{diag}\left(\epsilon_{1}, \ldots, \epsilon_{n}\right)$ with $\left|\epsilon_{k}\right|=1$ such that $A={ }^{t} \bar{C} E C$. Then $X={ }^{t} \bar{C} D C$ for $D:=I_{n}-\lambda^{-1} E=\operatorname{diag}\left(1-\alpha_{1}, \ldots, 1-\alpha_{n}\right)$ with $\alpha_{i}:=\lambda^{-1} \epsilon_{i}$ (all of modulus 1 ). It follows that

$$
\begin{aligned}
\sum_{b \in B}\|T(b)-\lambda b\|^{2}=\operatorname{Tr}\left({ }^{t} \bar{X} \cdot X\right)=\operatorname{Tr}\left({ }^{t} \bar{C}^{t} \bar{D} C \cdot{ }^{t} \bar{C} D C\right)=\operatorname{Tr}\left({ }^{t} \bar{D} D\right) \\
=\sum_{l=1}^{n}\left|1-\alpha_{l}\right|^{2}=\sum_{l=1}^{n}\left(1+\left|\alpha_{l}\right|^{2}-2 \Re\left(\alpha_{l}\right)\right)=2 n-2 \Re\left(\sum_{l=1}^{n} \alpha_{l}\right) .
\end{aligned}
$$

In particular,

$$
\sum_{b \in B}\|T(b)-\lambda b\|^{2} \geq 2 n-2\left|\sum_{l=1}^{n} \alpha_{l}\right|=2 n-2\left|\sum_{l=1}^{n} \epsilon_{l}\right|=2(n-|\operatorname{Tr}(T)|) .
$$

Taking the infimum over all $\lambda, B$ we obtain $d_{2}(T)^{2} \geq 2(n-|\operatorname{Tr}(T)|)$.

Now, in the above computation we choose $\lambda=\lambda_{0}:=e^{i \theta}$, where $\operatorname{Tr}(T)=r e^{i \theta}$ and $|\operatorname{Tr}(T)|=r \geq 0$. Then

$$
\sum_{l=1}^{n} \alpha_{l}=\lambda_{0}^{-1} \sum_{l=1}^{n} \epsilon_{l}=\lambda_{0}^{-1} \operatorname{Tr}(T)=e^{-i \theta} r e^{i \theta}=r=|\operatorname{Tr}(T)| .
$$

Then (2) implies that

$$
d_{2}(T)^{2} \leq \sum_{b \in B}\left\|T(b)-\lambda_{0} b\right\|^{2}=2 n-2 \Re\left(\sum_{l=1}^{n} \alpha_{l}\right)=2(n-|\operatorname{Tr}(T)|) \leq d_{2}(T)^{2} .
$$

Together with (3), this last inequality chain yields

$$
d_{2}(T)^{2}=2(n-|\operatorname{Tr}(T)|)=\sum_{b \in B}\left\|T(b)-\lambda_{0} b\right\|^{2}=\inf _{\lambda \in S^{1}} \sum_{b \in B}\|T(b)-\lambda b\|^{2} .
$$

The relationship between $\|T\|$ and $d_{2}(T)$ can be described as follows: 
Corollary 2.12. For $T \in G U(V)$ one has $4 \inf _{\lambda \in S^{1}}\|\lambda T\|<d_{2}(T) \leq 2 \pi\|T\|$.

Proof. We may assume that $T=\operatorname{diag}\left(e^{2 \pi i a_{1}}, \ldots, e^{2 \pi i a_{n}}\right)$ in some basis $B \in \mathcal{B}(V)$, where $-1 / 2 \leq a_{j}<1 / 2$; in particular, $\|T\|=\left(\sum_{j=1}^{n} a_{j}^{2}\right)^{1 / 2}$. It is easy to check that the function $(1-\cos (x)) / x^{2}$ is decreasing on $(0, \pi]$, whence $2 / \pi^{2} \leq(1-\cos (x)) / x^{2} \leq 1 / 2$ for $-\pi \leq x \leq \pi$. Taking $x=2 \pi a_{j}$, we get $4 / \pi^{2}<\left|e^{2 \pi i a_{j}}-1\right|^{2} / 4 \pi^{2} a_{j}^{2} \leq 1$, whence $4\|T\| \leq\left(\sum_{b \in B}\|T(b)-b\|^{j}\right)^{1 / 2} \leq 2 \pi\|T\|$. Now the statement follows by applying this inequality to $\lambda T$ for all $\lambda \in S^{1}$ and using Lemma 2.11(iii).

Lemma 2.13. Let $T_{1}, \ldots, T_{k} \in G U(V)$. Then the following hold:

(i) $d_{2}\left(T_{1} \ldots T_{k}\right)^{2} \leq k \cdot \sum_{i=1}^{k} d_{2}\left(T_{i}\right)^{2}$.

(ii) $d_{2}\left(T_{1} T_{2} T_{1}^{-1} T_{2}^{-1}\right)^{2} \leq 4 \min \left\{d_{2}\left(T_{1}\right)^{2}, d_{2}\left(T_{2}\right)^{2}\right\}$.

Proof. (i) For any $\lambda_{i} \in S^{1}, B \in \mathcal{B}(V)$, and $b \in B$ we have

$$
\begin{array}{r}
T_{1} \ldots T_{k}(b)-\lambda_{1} \ldots \lambda_{k} b=T_{1} \ldots T_{k-1}\left(T_{k} b-\lambda_{1} b\right)+\lambda_{1} T_{1} \ldots T_{k-2}\left(T_{k-1} b-\lambda_{2} b\right) \\
+\lambda_{1} \lambda_{2} T_{1} \ldots T_{k-3}\left(T_{k-2} b-\lambda_{3} b\right)+\cdots+\lambda_{1} \ldots \lambda_{k-1}\left(T_{1} b-\lambda_{k} b\right) .
\end{array}
$$

By the Cauchy-Schwarz inequality, $\left\|\sum_{i=1}^{k} v_{i}\right\|^{2} \leq k \sum_{i=1}^{k}\left\|v_{i}\right\|^{2}$ for any $v_{1}, \ldots, v_{k} \in V$. Since $\left|\lambda_{i}\right|=1$ and $T_{i}$ is unitary for all $i$, it now follows that

$$
\left\|T_{1} \ldots T_{k}(b)-\lambda_{1} \ldots \lambda_{k} b\right\|^{2} \leq k \sum_{i=1}^{k}\left\|T_{i} b-\lambda_{i} b\right\|^{2},
$$

But $\lambda_{1} \ldots \lambda_{k} \in S^{1}$, hence $d_{2}\left(T_{1} \ldots T_{k}\right)^{2} \leq k \sum_{i=1}^{k} \sum_{b \in B}\left\|T_{i} b-\lambda_{i} b\right\|^{2}$. Taking the infimum over all $\lambda_{i} \in S^{1}$ and applying Lemma 2.11(iii), we obtain $d_{2}\left(T_{1} \ldots T_{k}\right)^{2} \leq$ $k \sum_{i=1}^{k} d_{2}\left(T_{i}\right)^{2}$.

(ii) By (i) applied to $S:=\left(T_{1} T_{2} T_{1}^{-1}\right) \cdot T_{2}^{-1}$ and by Lemma 2.10,

$$
d_{2}(S)^{2} \leq 2\left(d_{2}\left(T_{1} T_{2} T_{1}^{-1}\right)^{2}+d_{2}\left(T_{2}^{-1}\right)^{2}\right)=4 d_{2}\left(T_{2}\right)^{2} .
$$

Breaking up $S=T_{1} \cdot T_{2} T_{1}^{-1} T_{2}^{-1}$ and arguing similarly, we get $d_{2}(S)^{2} \leq 4 d_{2}\left(T_{1}\right)^{2}$.

Lemmas 2.11(iii) and 2.13 yield the following inequalities which we believe to be new and non-trivial.

Corollary 2.14. Let $\chi$ be any complex character of any finite group $G$ and let $g_{1}, \ldots, g_{k}$ $\in G$. Then

(i) $\left(k^{2}-1\right) \chi(1)-k \sum_{i=1}^{k}\left|\chi\left(g_{i}\right)\right|+\left|\chi\left(\prod_{i=1}^{k} g_{i}\right)\right| \geq 0$.

(ii) $3 \chi(1)-4\left|\chi\left(g_{i}\right)\right|+\left|\chi\left(\left[g_{1}, g_{2}\right]\right)\right| \geq 0$ for $i=1,2$.

Now we can prove an upper bound that links the dimension, covering number, and deviation together.

Lemma 2.15. Let $V=\mathbb{C}^{n}$ with $n>1, G<G L(V)$ a finite irreducible subgroup, and let $g \in G$. Assume that any element of $G / Z(G)$ is a product, of length at most $\boldsymbol{\beta}$, of conjugates of $\bar{g}=g Z(G)$. Then $\operatorname{dim}(V) \leq\left(\boldsymbol{\beta} d_{2}(g)\right)^{2} / 2$. 
Proof. By Weyl's unitarian trick we can equip $V$ with a $G$-invariant Hermitian form and assume $G<G U(V)$. Consider any element $h \in G \backslash Z(G)$. Then $h=g_{1} \ldots g_{k} z$ with $g_{i} \in g^{G}, z \in Z(G)$, and $k \leq \boldsymbol{\beta}$. By Schur's Lemma, $z$ is scalar, hence $d_{2}(h)=d_{2}\left(h^{\prime}\right)$ for $h^{\prime}:=g_{1} \ldots g_{k}$ by Lemma 2.10(i). Next, by Lemmas 2.10(ii) and 2.13(i), $d_{2}\left(h^{\prime}\right)^{2} \leq$ $k \sum_{i=1}^{k} d_{2}\left(g_{i}\right)^{2}=k^{2} d_{2}(g)^{2}$. It follows that $d_{2}(h)^{2} \leq\left(\boldsymbol{\beta} d_{2}(g)\right)^{2}$.

Now by Burnside's theorem on zeros we can choose $h$ such that $\operatorname{Tr}(h)=0$. By Lemma 2.11(iii), $d_{2}(h)^{2}=2 n$ and so $2 n \leq\left(\beta d_{2}(g)\right)^{2}$.

Recall that a finite group $G$ is almost quasi-simple if $S \triangleleft G / Z(G) \leq \operatorname{Aut}(S)$ for some finite non-abelian simple group $S$. For any such $S$ and any $x \in S$, let $\boldsymbol{\alpha}(x)$ be the minimal number of $\operatorname{Aut}(S)$-conjugates of $x$ which generate the subgroup $\langle S, x\rangle$. A sharp upper bound on $\boldsymbol{\alpha}(x)$ for $1 \neq x \in \operatorname{Aut}(S)$ has been obtained in [GS]. We will need the following result of [GT2] that uses $\boldsymbol{\alpha}(x)$ to bound the dimension of eigenspaces:

Lemma 2.16 ([GT2, Lemma 3.2]). Let $G$ be a finite almost quasi-simple group acting faithfully and irreducibly on a finite-dimensional vector space $V$ over a field $\mathbb{F}$, and let $g \in G \backslash Z(G)$. Then the dimension of any eigenspace of $g$ on $V$ is at most $\operatorname{dim}(V)-$ $\operatorname{dim}(V) / \boldsymbol{\alpha}(g Z(G))$.

Next we prove key inequalities which relate the age of any element $g \in G U(V)$ to its deviations.

Proposition 2.17. Let $g \in G U(V)$ and let $X$ be a non-empty subset of eigenvalues of $g$. Let $m \geq 1$ be such that any $\lambda \in X$ occurs as an eigenvalue of $g$ on $V$ with multiplicity at least $m$. Also assume that the shortest arc of $S^{1}$ that contains $X$ has length $\geq \delta>0$. Then

(i) $2 \pi \cdot \operatorname{age}(g)-d_{1}(g) \geq m(\delta-2 \sin (\delta / 2))$; in particular, $d_{1}(g) \leq 2 \pi \cdot \operatorname{age}(g)$.

(ii) $4 \pi \cdot \operatorname{age}(g)-d_{2}(g)^{2} \geq 2 m(\delta-1+\cos (\delta))$.

(iii) $d_{2}(g)^{2} \leq(2.9) \pi \cdot \operatorname{age}(g)$. In fact, if $\delta \geq \pi-\sin ^{-1}(0.725)$ then

$$
\text { (2.9) } \pi \cdot \operatorname{age}(g)-d_{2}(g)^{2} \geq m\{(1.45) \delta-2(1-\cos (\delta))\} .
$$

Proof. Let $g$ be represented by $\operatorname{diag}\left(\epsilon_{1}, \ldots, \epsilon_{n}\right)$ in a basis $B_{0} \in \mathcal{B}(V)$, where $\epsilon_{j}=e^{2 \pi i r_{j}}$, $0 \leq r_{j}<1$; in particular, age $(g)=\sum_{j=1}^{n} r_{j}$.

(i) Consider the function $f(x):=2 \pi x-\left|e^{2 \pi i x}-1\right|=2 \pi x-2 \sin (\pi x)$ on $[0, \infty)$. Since $f^{\prime}(x)=2 \pi-2 \pi \cos (\pi x) \geq 0, f$ is increasing; also, $f(x) \geq 0$. Taking $\lambda=1$ and $B=B_{0}$ in the proof of Lemma 2.11(ii), we see that $d_{1}(g) \leq \sum_{j=1}^{n}\left|\epsilon_{j}-1\right|$. Hence

$$
2 \pi \cdot \operatorname{age}(g)-d_{1}(g) \geq \sum_{j=1}^{n} f\left(r_{j}\right) \geq \sum_{j: \epsilon_{j} \in X} f\left(r_{j}\right) .
$$

Suppose that $0 \leq r_{j} \leq \delta^{\prime} / 2 \pi<\delta / 2 \pi$ for all $\epsilon_{j} \in X$. Then $X$ is contained in the arc (from 1 to $e^{i \delta^{\prime}}$ ) of length $\delta^{\prime}<\delta$, contrary to the assumption. So without loss we may assume that $\epsilon_{1} \in X$ and $r_{1} \geq \delta / 2 \pi$. Since $\epsilon_{1}$ occurs as an eigenvalue of $g$ with multiplicity $\geq m$, we get

$$
2 \pi \cdot \operatorname{age}(g)-d_{1}(g) \geq \sum_{j: \epsilon_{j} \in X} f\left(r_{j}\right) \geq m f\left(r_{1}\right) \geq m f(\delta / 2 \pi)=m(\delta-2 \sin (\delta / 2)) .
$$


(ii) Consider the function $h(x):=4 \pi x-\left|e^{2 \pi i x}-1\right|^{2}=4 \pi x-2(1-\cos (2 \pi x))$ on $[0, \infty)$. Since $h^{\prime}(x)=4 \pi(1-\sin (2 \pi x)) \geq 0, h$ is increasing, whence $h(x) \geq h(0)=0$. Taking $\lambda=1$ and $B=B_{0}$ in the proof of Lemma 2.11(iii), we see that $d_{2}(g)^{2} \leq$ $\sum_{j=1}^{n}\left|\epsilon_{j}-1\right|^{2}$. Hence

$$
4 \pi \cdot \operatorname{age}(g)-d_{2}(g)^{2} \geq \sum_{j=1}^{n} h\left(r_{j}\right) \geq \sum_{j: \epsilon_{j} \in X} h\left(r_{j}\right) .
$$

As in (i), we may assume without loss that $\epsilon_{1} \in X$ and $r_{1} \geq \delta / 2 \pi$. Since $\epsilon_{1}$ occurs as an eigenvalue of $g$ with multiplicity $\geq m$, we get

$$
4 \pi \cdot \operatorname{age}(g)-d_{2}(g)^{2} \geq \sum_{j: \epsilon_{j} \in X} h\left(r_{j}\right) \geq m h\left(r_{1}\right) \geq m h(\delta / 2 \pi)=2 m(\delta-1+\cos (\delta)) .
$$

(iii) Consider the function $t(x):=(1.45) x-\left|e^{i x}-1\right|^{2}=(1.45) x-2(1-\cos (x))$ on $[0,2 \pi]$. Since $t^{\prime}(x)=1.45-2 \sin (x), t$ is increasing on $[0, \theta] \cup[\pi-\theta, 2 \pi]$ and decreasing on $[\theta, \pi-\theta]$, where $\theta:=\sin ^{-1}(0.725)$. Now $t(0)=0$ and $t(\pi-\theta)>0.0018$, and so $t(x) \geq 0$ on $[0,2 \pi]$. As above, $d_{2}(g)^{2} \leq \sum_{j=1}^{n}\left|\epsilon_{j}-1\right|^{2}$, hence

$$
\text { (2.9) } \pi \cdot \operatorname{age}(g)-d_{2}(g)^{2} \geq \sum_{j=1}^{n} t\left(2 \pi r_{j}\right) \geq 0 .
$$

Next suppose that $\delta \geq \pi-\theta$. As in (i), we may assume without loss that $\epsilon_{1} \in X$ and $r_{1} \geq \delta / 2 \pi$. Since $\epsilon_{1}$ occurs as an eigenvalue of $g$ with multiplicity $\geq m, t(x) \geq 0$ and $t$ is increasing on $[\pi-\theta, 2 \pi]$, we see that $(2.9) \pi \cdot \operatorname{age}(g)-d_{2}(g)^{2} \geq m \cdot t\left(2 \pi r_{1}\right) \geq m \cdot t(\delta)$.

Proposition 2.17 yields the following immediate consequence:

Corollary 2.18. Let $G<G L(V)$ be a finite subgroup. Assume $g \in G$ is such that age* $(g) \leq 1$. Then $d_{1}(g) \leq 2 \pi$ and $d_{2}(g)^{2} \leq(2.9) \pi<9.111$. Furthermore, $\operatorname{dim}(V)-$ $|\operatorname{Tr}(g)| \leq(1.45) \pi<4.556$. In fact,

$$
\operatorname{dim}(V)-|\operatorname{Tr}(g)|< \begin{cases}4.278 & \text { if } \delta \geq \pi, \\ 3.632 & \text { if } \delta \geq 6 \pi / 5, \\ 3.019 & \text { if } \delta \geq 4 \pi / 3 \\ 2.676 & \text { if } \delta \geq 7 \pi / 5 \\ 2.139 & \text { if } \delta \geq 3 \pi / 2\end{cases}
$$

where $\delta$ is the length of the shortest arc of $S^{1}$ that contains all eigenvalues of $g$.

Proof. We apply Lemma 2.11(iii), and Proposition 2.17(i), (iii), with $X=\operatorname{Spec}(\lambda g, V)$ for any $\lambda \in S^{1}$. Then the claims follow by taking the infimum over all $\lambda \in S^{1}$.

Taking $g=\operatorname{diag}(1,1, \ldots, 1,-1,-1) \in G L_{n}(\mathbb{C})$ with $n \geq 4$, we see that age $(g)=1$ and $d_{2}(g)^{2}=8$. In fact, the complex reflection $g=\operatorname{diag}\left(e^{2 \pi i / 3}, 1, \ldots, 1\right) \in G L_{n}(\mathbb{C})$ has age $(g)=1 / 3$ and $d_{2}(g)^{2}=2\left(n-\sqrt{n^{2}-3 n+3}\right)$. Hence, when $n \rightarrow \infty, d_{2}(g)^{2} \rightarrow 3$, yielding $d_{2}(g)^{2} / \operatorname{age}(g) \rightarrow 9$. Thus the constant $(2.9) \pi \approx 9.111$ in Proposition 2.17 (iii) and Corollary 2.18 is quite good. 
Lemma 2.19. Let $G<G L(V)$ be irreducible, primitive, and tensor indecomposable on $V$, with a normal subgroup $L \triangleleft G$ such that $L^{\prime} \nless \leq Z(G)$.

(i) For any non-scalar $g \in G$, there exists $h \in L \backslash Z(L)$ such that $d_{2}(h)^{2} \leq 4 d_{2}(g)^{2}$.

(ii) Assume furthermore that $\operatorname{dim}(V)>1$ and that $G$ satisfies the set-up $(\star)$. Then there exists $h \in L \backslash Z(L)$ such that $d_{2}(h)^{2}<36.444$.

Proof. (i) By [GT3, Lemma 2.5], $L^{\prime}$ is irreducible on $V$. We claim that there exists $u \in L$ such that $[g, u] \notin Z(L)$. Assume the contrary: $[g, u] \in C_{G}(L)$ for any $u \in L$. Then for any $u, v \in L$ we have $[[u, v], g]=([[v, g], u] \cdot[[g, u], v])^{-1}=1$, whence $\left[g, L^{\prime}\right]=1$. By Schur's Lemma, the irreducibility of $L^{\prime}$ on $V$ now implies that $g$ is scalar, a contradiction. Now we define $h=[g, u] \in L \backslash Z(L)$, and we are done by Lemma 2.13(ii).

(ii) If every $g \in \mathcal{X}$ acts scalarly on $V$, then so does $G$. But in this case $\operatorname{dim}(V)=1$, a contradiction. Hence at least one $g \in \mathcal{X}$ is non-scalar, and has age $\leq 1$. Now the claim follows from (i) and Corollary 2.18.

\subsection{Elements of small order}

To estimate the age of elements of small order, we will need the following two statements.

Lemma 2.20. Assume $g \in G U(V)$ is conjugate to

$$
\operatorname{diag}\left(\alpha_{1},-\alpha_{1}, \alpha_{2},-\alpha_{2}, \ldots, \alpha_{m},-\alpha_{m}, \beta_{1}, \ldots, \beta_{s}\right) .
$$

Then age* $(g) \geq m / 2$. Moreover, if age ${ }^{*}(g)=m / 2$ then $g$ has exactly two distinct eigenvalues.

Proof. Suppose age* $(g) \leq m / 2$. Then age $(\mu g) \leq m / 2$ for some $\mu \in S^{1}$ by Lemma 2.2(ii). Note that the contribution of the pair $\left(\mu \alpha_{i},-\mu \alpha_{i}\right)$ to age $(g)$ is at least $1 / 2$, and it equals $1 / 2$ precisely when $\alpha_{i}= \pm \mu^{-1}$. Next, the contribution of $\mu \beta_{j}$ to age $(g)$ is at least 0 , and it equals 0 precisely when $\beta_{j}=\mu^{-1}$. Hence the statements follow.

Lemma 2.21. Let $g \in G U(V)$ be a non-scalar element of age $\leq 1, \operatorname{dim}(V) \geq 4$, and let $\lambda g$ have order $1<m \leq 5$ for some $\lambda \in S^{1}$. Then there is some $\mu \in S^{1}$ such that either $\mu \mathrm{g}$ is a complex reflection, or one of the following statements holds for a suitable choice of $i=\sqrt{-1}$.

(i) $m=2$, and $g$ is a bireflection.

(ii) $m=3$, and one of the following holds, where $\omega=e^{2 \pi i / 3}$.

(a) $\mu g$ is conjugate to $\operatorname{diag}(\omega, \omega, 1, \ldots, 1)$.

(b) age $(g)=1$, and $g$ is conjugate to

$$
\operatorname{diag}\left(\omega, \omega^{2}, 1, \ldots, 1\right) \quad \text { or } \quad \operatorname{diag}(\omega, \omega, \omega, 1, \ldots, 1) .
$$

(iii) $m=4$, and one of the following holds.

(a) $\mu \mathrm{g}$ is conjugate to one of the elements

$$
\operatorname{diag}(i, i, 1, \ldots, 1), \quad \operatorname{diag}(i, i, i, 1, \ldots, 1), \quad \operatorname{diag}(i,-1,1, \ldots, 1) .
$$


(b) age $(g)=1$, and $g$ is conjugate to one of the elements

$$
\begin{aligned}
\operatorname{diag}(i,-i, 1, \ldots, 1), \quad & \operatorname{diag}(-1,-1,1, \ldots, 1), \quad \operatorname{diag}(i, i,-1,1, \ldots, 1), \\
& \operatorname{diag}(i, i, i, i, 1, \ldots, 1) .
\end{aligned}
$$

(iv) $m=5$, and one of the following holds, where $\epsilon=e^{2 \pi i / 5}$.

(a) $\mu \mathrm{g}$ is conjugate to one of the elements

$$
\begin{gathered}
\operatorname{diag}(\epsilon, \epsilon, 1, \ldots, 1), \quad \operatorname{diag}\left(\epsilon^{2}, \epsilon^{2}, 1, \ldots, 1\right), \\
\operatorname{diag}(\epsilon, \epsilon, \epsilon, 1, \ldots, 1), \quad \operatorname{diag}(\epsilon, \epsilon, \epsilon, \epsilon, 1, \ldots, 1), \\
\operatorname{diag}\left(\epsilon, \epsilon^{2}, 1, \ldots, 1\right), \quad \operatorname{diag}\left(\epsilon, \epsilon^{3}, 1, \ldots, 1\right), \quad \operatorname{diag}\left(\epsilon, \epsilon, \epsilon^{2}, 1, \ldots, 1\right) .
\end{gathered}
$$

(b) $\operatorname{age}(g)=1$, and $g$ is conjugate to one of the elements

$$
\begin{gathered}
\operatorname{diag}\left(\epsilon, \epsilon^{4}, 1, \ldots, 1\right), \quad \operatorname{diag}\left(\epsilon^{2}, \epsilon^{3}, 1, \ldots, 1\right), \quad \operatorname{diag}\left(\epsilon, \epsilon^{2}, \epsilon^{2}, 1, \ldots, 1\right), \\
\operatorname{diag}\left(\epsilon, \epsilon, \epsilon^{3}, 1, \ldots, 1\right), \quad \operatorname{diag}\left(\epsilon, \epsilon, \epsilon, \epsilon^{2}, 1, \ldots, 1\right), \quad \operatorname{diag}(\epsilon, \epsilon, \epsilon, \epsilon, \epsilon, 1, \ldots, 1) .
\end{gathered}
$$

Proof. The proofs of all these statements are similar, and we only handle (iv). By the assumption, there is some $t \in[0,1 / 5)$ and integers $a, b, c, d, e \geq 0$ such that $a+b+c+$ $d+e=\operatorname{dim}(V) \geq 4$ and

$$
1 \geq \operatorname{age}(g)=a t+b(t+1 / 5)+c(t+2 / 5)+d(t+3 / 5)+e(t+4 / 5) ;
$$

in particular, $b+2 c+3 d+4 e \leq 5$. Now (iv) follows by an exhaustive enumeration.

\subsection{Character ratios}

We will need the following result of Gluck and Magaard [G], [GM].

Proposition 2.22. Let $G$ be a finite group, let $\chi \in \operatorname{Irr}(G)$ be of degree $>1$, and let $g \in G \backslash Z(G)$.

(i) ([GM, Theorem 2.4]) Assume $G$ is a finite quasi-simple group, not $\mathrm{A}_{n}$ nor $2 \mathrm{~A}_{n}$ with $n \geq 10$. Then $|\chi(g) / \chi(1)| \leq 19 / 20$.

(ii) ([GM, Theorem 1.6]) Let $G=\mathrm{S}_{n}$ or $\mathrm{A}_{n}$ with $n \geq 5$, and let $c(g)$ be the number of cycles of the permutation $g$. Then $|\chi(g) / \chi(1)| \leq 1 / 2+c(g) / 2 n$.

Next we address the character ratios for spin representations of $2 A_{n}$ and $2 S_{n}$.

Lemma 2.23. Let $G=2 S_{n}$ or $2 \mathrm{~A}_{n}$ with $n \geq 6, \chi \in \operatorname{Irr}(G)$ a faithful character of $G$, and let $g \in G \backslash Z(G)$. Then $|\chi(g) / \chi(1)| \leq 7 / 8$.

Proof. Since $g \notin Z(G)=C_{G}\left(G^{\prime}\right)$ and since $G^{\prime}=2 \mathrm{~A}_{n}$ is generated by commutators $[x, y]$ with $x, y$ being inverse images of 3-cycles, there exists an inverse image $t$ of a 3cycle such that $h:=[g, t] \notin Z(G)$. Observe that $h=g t g^{-1} \cdot t^{-1}$ projects onto the product of two 3-cycles. It follows that (a $G$-conjugate of) $h$ is contained in a natural subgroup $K \cong 2 \mathrm{~A}_{6}$ of $G$. (See [GM, Lemma 2.5] for a similar argument.) Clearly, $h \notin Z(K)$ since $[g, t] \notin Z(G)$. Also, the restriction $\left.\chi\right|_{K}$ is a sum of faithful irreducible characters of $K$. Inspecting [Atlas], one can check that $|\chi(h)| \leq \chi(1) / 2$, and so $d_{2}(h)^{2} \geq \chi(1)$. It now follows from Lemma 2.13(ii) that $d_{2}(g)^{2} \geq \chi(1) / 4$, whence $|\chi(g) / \chi(1)| \leq 7 / 8$ by Lemma 2.11(iii). 


\subsection{Tensor decomposable and tensor induced modules}

First we recall a well-known remark:

Lemma 2.24. Let $G$ be a finite irreducible subgroup of $G L(W)$. Assume that there is a tensor decomposition $W=U \otimes V$ such that $G<G L(U) \otimes G L(V)$. Then there is a finite central extension $1 \rightarrow Z \rightarrow \tilde{G} \rightarrow G \rightarrow 1$ of $G$ and irreducible representations $\Phi: \tilde{G} \rightarrow G L(U)$ and $\Psi: \tilde{G} \rightarrow G L(V)$ such that $g=\Phi(\tilde{g}) \otimes \Psi(\tilde{g})$ for any $g=\tilde{g} Z \in G$.

Proof. First we observe that if $a \otimes b=c \otimes d$ for some $a, c \in G L(U)$ and $b, d \in G L(V)$, then there is some $\gamma \in \mathbb{C}^{\times}$such that $a=\gamma c$ and $b=\gamma^{-1} d$. Now, by hypothesis, there are maps $A: G \rightarrow G L(U)$ and $B: G \rightarrow G L(V)$ such that $g=A(g) \otimes B(g)$ for any $g \in G$. If $h \in G$, then

$A(g h) \otimes B(g h)=g h=(A(g) \otimes B(g)) \cdot(A(h) \otimes B(h))=(A(g) \cdot A(h)) \otimes(B(g) \cdot B(h))$.

By our observation, we see that $A(g h)=\lambda(g, h) A(g) A(h)$ for some 2-cocycle $\lambda: G \times G$ $\rightarrow \mathbb{C}^{\times}$, and so $A$ is a projective (irreducible) representation of $G$. Thus $A$ lifts to a linear representation $\Phi: \tilde{G} \rightarrow G L(U)$ of a finite central extension $\tilde{G}$ of $G: A(g)=$ $\alpha(\tilde{g}) \Phi(\tilde{g})$, where $\alpha: \tilde{G} \rightarrow \mathbb{C}^{\times}$and $g=\tilde{g} Z$. Now it is easy to check that the map $\Psi: \tilde{G} \rightarrow G L(V)$ defined by $\Psi(\tilde{g})=\alpha(\tilde{g}) B(g)$ for $g=\tilde{g} Z$ is a group homomorphism, and $g=\Phi(\tilde{g}) \otimes \Psi(\tilde{g})$.

Lemma 2.24 shows that if a finite irreducible subgroup $G$ of $G L(V)$ preserves a tensor decomposition of $V$, then we may (and will) view $V$ as the tensor product of two modules for some central extension $\tilde{G}$ of $G$, and then replace $G$ by $\tilde{G}$.

Let $V=\mathbb{C}^{d}$ be a $G$-module which is tensor induced. This means that there is a tensor decomposition $V=V_{1}^{\otimes m}$ such that (the action of) $G$ (on $V$ ) is contained in $G L\left(V_{1}\right)^{\otimes m}: \mathrm{S}_{m}$, with $\mathrm{S}_{m}$ naturally permuting the $m$ tensor factors of $V$. (Note that we do not claim that $G \leq H^{\otimes m}: \mathrm{S}_{m}$ for a finite subgroup $H \in G L\left(V_{1}\right)$.)

Lemma 2.25. Under the above assumptions, assume $G$ is finite and $g \in G$ projects onto $h \in \mathrm{S}_{m}$, a product of s disjoint cycles. Then $|\operatorname{Tr}(g)| \leq \operatorname{dim}\left(V_{1}\right)^{s}$.

Proof. First we observe that if $y=a \otimes b$ has finite order for $a \in G L(U)$ and $b \in G L(V)$, then there is some $\delta \in \mathbb{C}^{\times}$such that both $c:=\delta^{-1} a$ and $d:=\delta b$ have finite order, and $y=c \otimes d$. (Indeed, $I=y^{N}=a^{N} \otimes b^{N}$, where we use $I$ to denote any identity matrix. So by the first sentence of the proof of Lemma 2.24, $a^{N}=\gamma I$ and $b^{N}=\gamma^{-1} I$ for some $\gamma \in \mathbb{C}^{\times}$and $0<N \in \mathbb{Z}$. Now choose $\delta$ to be an $N^{\text {th }}$ root of $\gamma$.)

In the case $s>1$, conjugating $g$ with a suitable element in $\mathrm{S}_{m}$ we may assume that $g$ preserves a tensor decomposition of $V$. Using the above observation and proceeding by induction on $s$, we may assume that $s=1$ and $h=(1,2, \ldots, m)$. Now $g=h b$ with $b=B_{1} \otimes \ldots \otimes B_{m}$ and $B_{i} \in G L\left(V_{1}\right)$. Then one can check (see also [GI]) that $\operatorname{Tr}(g)=\operatorname{Tr}\left(B_{1} \ldots B_{m}\right)$. Since $G$ and $\mathrm{S}_{m}$ are finite, there is some integer $N>1$ such that $g^{N}=h^{N}=$ Id. Since $I=h^{N}=g^{N}=h^{N} b^{h^{N-1}} b^{h^{N-2}} \ldots b^{h} b$ (where $b^{x}:=x^{-1} b x$ ), we have

$$
I=g^{N}=\left(B_{2} \ldots B_{m} B_{1}\right)^{N / m} \otimes\left(B_{3} \ldots B_{m} B_{1} B_{2}\right)^{N / m} \otimes \cdots \otimes\left(B_{1} \ldots B_{m}\right)^{N / m} .
$$


Pick an arbitrary eigenvalue $\lambda$ of $v:=B_{1} \ldots B_{m}$. Note that all the matrices $B_{2} \ldots B_{m_{1}} B_{1}$, $B_{3} \ldots B_{m_{1}} B_{1} B_{2}, \ldots$ are conjugate to $v$. Hence $\underbrace{\lambda^{N / m} \ldots \lambda^{N / m}}_{m}=\lambda^{N}$ is an eigenvalue of $g^{N}=I$. We have shown that each eigenvalue of $v$ is an $N^{\text {th }}$ root of unity and so it has absolute value 1 . Hence $|\operatorname{Tr}(g)|=|\operatorname{Tr}(v)|$ is at most the size of $v$, which is $\operatorname{dim}\left(V_{1}\right)$.

We will also need the following technical statement:

Lemma 2.26. Let $\mathcal{C}$ be a collection of finite simple groups and let $G$ be any finite group. Then $G$ has a unique normal subgroup $R$ such that

(i) every composition factor of $R$ belongs to $\mathcal{C}$; and

(ii) if $N \triangleleft G$ and every composition factor of $N$ belongs to $\mathcal{C}$, then $N \leq R$.

Furthermore, $R$ is a characteristic subgroup of $G$.

Proof. Let $\mathcal{X}$ be the collection of all normal subgroups $N \triangleleft G$ with the property that all composition factors of $N$ belong to $\mathcal{C}$. For any $M, N \in \mathcal{X}, M N \triangleleft G$, and every composition factor of $M N$ also belongs to $\mathcal{C}$ since $M N / N \cong M /(M \cap N)$, whence $M N \in \mathcal{X}$. Now the subgroup $R=\prod_{N \in \mathcal{X}} N$ clearly satisfies (i) and (ii). Let $\varphi \in \operatorname{Aut}(G)$. Then $\varphi(R) \triangleleft G$ and $\varphi(R) \in \mathcal{X}$ since $\varphi(R) \cong R$. By (ii), $\varphi(R)=R$.

\section{Proof of Theorem 1.3}

\subsection{Reduction to the almost quasi-simple case}

Proposition 3.1. It suffices to prove Theorem 1.3 for the case where $G$ is an almost quasisimple group which is irreducible, primitive, tensor indecomposable, and not tensor induced on $V$.

Proof. Let $\chi$ denote the character of $G$ afforded by $V$.

(i) First we consider the case $G$ is tensor induced on $V: V=V_{1} \otimes \cdots \otimes V_{m}$, with $\operatorname{dim}\left(V_{i}\right)=a>1$ and $G$ permutes the $m$ tensor factors $V_{1}, \ldots, V_{m}$ (transitively). By assumption, $g$ acts non-trivially on the set $\left\{V_{1}, \ldots, V_{m}\right\}$. Hence, $|\chi(g)| \leq a^{m-1} \leq d / 2$ by Lemma 2.25. Now if $d=a^{m} \geq 8$ then $\Delta(g) \geq d / 2 \geq 4$. On the other hand, if $d=a^{m}<8$, then $d=4$ and $\Delta(g) \geq d / 2=2$.

(ii) Now assume that we are in the extraspecial case (i.e. the case (iii) of [GT3, Proposition 2.8]). Then $d=p^{m}$ for some prime $p$ and some integer $m \geq 2$. By [GT1, Lemma 2.4], $|\chi(g)| \leq p^{m-1 / 2} \leq d / \sqrt{2}$. In particular, if $d \geq 8$, then $\Delta(g) \geq d(1-1 / \sqrt{2})$ $\geq 8-4 \sqrt{2}$. If $d=5$ or 7 , then $\Delta(g) \geq d-\sqrt{d} \geq 5-\sqrt{5}>8-4 \sqrt{2}$. If $d=4$, then $\Delta(g) \geq 4(1-1 / \sqrt{2})=4-2 \sqrt{2}$. If $d=3$, then $\Delta(g) \geq 3-\sqrt{3}$, and if $d=2$, then $\Delta(g) \geq 2-\sqrt{2}>(3-\sqrt{5}) / 2$.

(iii) Next we consider the tensor decomposable case: $V=V_{1} \otimes \cdots \otimes V_{m}$, where $G$ is tensor indecomposable and primitive on $V_{i}, \operatorname{dim}\left(V_{i}\right) \geq 2$, and $m \geq 2$. By [GT3, Proposition 2.8] and by the hypothesis, we may assume that Theorem 1.3 holds for $g$ acting on $V_{i}$ as long as $\left.g\right|_{V_{i}}$ is not scalar. Let $\alpha_{i}$ be the character afforded by $V_{i}$. 
Suppose that there is some $j$ such that $\operatorname{dim}\left(V_{j}\right) \geq 3$ and $\left.g\right|_{V_{j}}$ is non-scalar. Then $\alpha_{j}(1)-\left|\alpha_{j}(g)\right| \geq 4-\sqrt{8}$ by Theorem 1.3 applied to $\left(G, g, V_{j}\right)$. Hence

$\chi(1)-|\chi(g)| \geq \chi(1)-\frac{\chi(1)}{\alpha_{j}(1)}\left|\alpha_{j}(g)\right|=\frac{\chi(1)}{\alpha_{j}(1)}\left(\alpha_{j}(1)-\left|\alpha_{j}(g)\right|\right) \geq 2(4-\sqrt{8})=8-4 \sqrt{2}$,

as required. So we may assume that $\operatorname{dim}\left(V_{i}\right)=2$ whenever $\left.g\right|_{V_{i}}$ is non-scalar. But $g$ is non-scalar, so without loss we may suppose that $\operatorname{dim}\left(V_{1}\right)=2$ and $\left.g\right|_{V_{1}}$ is non-scalar. By Theorem 1.3 applied to $\left(G, g, V_{1}\right)$ we have $\alpha_{1}(1)-\left|\alpha_{1}(g)\right| \geq(3-\sqrt{5}) / 2$. Arguing as above, we obtain

$$
\Delta(g)=\chi(1)-|\chi(g)| \geq \frac{\chi(1)}{\alpha_{1}(1)}\left(\alpha_{1}(1)-\left|\alpha_{1}(g)\right|\right) \geq d(3-\sqrt{5}) / 4 .
$$

If $d \geq 13$ in addition, then in fact $d \geq 14$ and $\Delta(g) \geq 7(3-\sqrt{5}) / 2>8-4 \sqrt{2}$. If $d=6$ or 10 , then $m=2, \operatorname{dim}\left(V_{2}\right)=3$ or 5 , and so $\left.g\right|_{V_{2}}$ is scalar, whence we arrive at the conclusion (v) of Theorem 1.3. The same holds if $m=2$ and $d \in\{8,12\}$. We also arrive at the same conclusion when $d=4$, as otherwise $\Delta(g) \geq 4-((1+\sqrt{5}) / 2)^{2}>4-\sqrt{8}$. Finally, consider the case where $d \in\{8,12\}$ but $m>2$; that is, $m=3$. Then we may assume that $\operatorname{dim}\left(V_{2}\right)=2$ and $\left.g\right|_{V_{2}}$ is not scalar (as otherwise Theorem 1.3(v) holds). As in the case $d=4$, we get $\alpha_{1}(1) \alpha_{2}(1)-\left|\alpha_{1}(g) \alpha_{2}(g)\right|>4-\sqrt{8}$, whence

$$
\Delta(g)=\chi(1)-|\chi(g)| \geq \frac{\chi(1)}{\alpha_{1}(1) \alpha_{2}(1)}\left(\alpha_{1}(1) \alpha_{2}(1)-\left|\alpha_{1}(g) \alpha_{2}(g)\right|\right)>8-4 \sqrt{2} .
$$

We are done by [GT3, Proposition 2.8].

Throughout the rest of this section we will assume that $G$ is an almost quasi-simple group. In fact, we will prove more than we need for the proof of Theorem 1.3: we will describe all triples $(G, V, g)$, where

(a) $G<G L(V)$ is an almost quasi-simple, irreducible, primitive, tensor indecomposable subgroup, $g \in G \backslash Z(G)$, and either $0<\operatorname{age}^{*}(g) \leq 1$, or $\Delta(g):=\operatorname{dim}(V)-$ $|\operatorname{Tr}(g)| \leq 8-4 \sqrt{2}$.

As usual, we denote by $\chi$ the character of $G$ afforded by $V, L:=G^{(\infty)}, S:=L / Z(L)$. The set-up (\%) implies that $\left.\chi\right|_{L}$ is irreducible, and that

$$
\Delta(g)=\chi(1)-|\chi(g)|<4.556
$$

by Corollary 2.18 .

\subsection{Alternating groups}

First we dispose of the case $S=\mathrm{A}_{n}$ with $n \geq 8$. For $1 \leq k \leq n-1$, let $R_{n}(k)$ denote the set of partitions $\lambda \vdash n$, where either $\lambda$ or the conjugate partition $\lambda^{*}$ has the form $(n-k, \mu)$ for some $\mu \vdash k$. We will need the following statement which follows from the main result of [Ra]: 
Lemma 3.2. Let $\rho=\rho^{\lambda} \in \operatorname{Irr}\left(\mathrm{S}_{n}\right)$ be labeled by the partition $\lambda \vdash n$.

(i) If $n \geq 15$, then either $\rho(1) \geq n(n-1)(n-5) / 6$, or $\lambda \in \bigcup_{k=1}^{2} R_{n}(k)$.

(ii) If $n \geq 22$, then either $\rho(1) \geq n(n-1)(n-2)(n-7) / 24$, or $\lambda \in \bigcup_{k=1}^{3} R_{n}(k)$.

We will now estimate $\rho^{\lambda}(g)$. Let $\mathbf{t}$ denote the transposition $(1,2) \in \mathrm{S}_{n}$.

Lemma 3.3. Let $1+\alpha(g)$ denote the number of fixed points of the permutation $g \in \mathrm{S}_{n}$, and let $n \geq 9$. Then

$$
\rho^{\lambda}(g)= \begin{cases}\left(\alpha(g)^{2}-\alpha\left(g^{2}\right)\right) / 2, & \lambda=\left(n-2,1^{2}\right), \\ \left(\alpha(g)^{2}+\alpha\left(g^{2}\right)\right) / 2-\alpha(g)-1, & \lambda=(n-2,2), \\ \left(\alpha(g)^{3}-3 \alpha(g) \alpha\left(g^{2}\right)+2 \alpha\left(g^{3}\right)\right) / 6, & \lambda=\left(n-3,1^{3}\right), \\ \left(\alpha(g)^{3}-\alpha\left(g^{3}\right)\right) / 3-\alpha(g)^{2}+1, & \lambda=(n-3,2,1), \\ \left(\alpha(g)^{3}+3 \alpha(g) \alpha\left(g^{2}\right)+2 \alpha\left(g^{3}\right)\right) / 6-\alpha(g)^{2}-\alpha(g), & \lambda=(n-3,3) .\end{cases}
$$

In particular, if $g \neq 1$ then $\left|\rho^{\lambda}(g)\right| \leq \rho^{\lambda}(\mathbf{t})$ for any of the above $\lambda$.

Proof. It is well known that $\operatorname{Sym}^{2}(\alpha)=\rho^{(n-2,2)}+\rho^{(n-1,1)}+\rho^{(n)}, \wedge^{2}(\alpha)=\rho^{\left(n-2,1^{2}\right)}$, and $\wedge^{3}(\alpha)=\rho^{\left(n-3,1^{3}\right)}$ (cf. [FH] for instance). Using the Littlewood-Richardson rule, one can see that

$$
\begin{aligned}
& \left(\operatorname{Ind}_{S_{n-1}}^{S_{n}}\left(\rho^{(n-1)}\right)\right) \otimes \wedge^{2}(\alpha)=\operatorname{Ind}_{S_{n-1}} S_{n}\left(\left(\rho^{\left(n-2,1^{2}\right)}\right) \mid S_{n-1}\right) \\
& =\operatorname{Ind}_{S_{n-1}}^{S_{n}}\left(\rho^{\left(n-3,1^{2}\right)}+\rho^{(n-2,1)}\right)=\rho^{\left(n-3,1^{3}\right)}+\rho^{(n-3,2,1)}+2 \rho^{\left(n-2,1^{2}\right)}+\rho^{(n-2,2)}+\rho^{(n-1,1)}
\end{aligned}
$$

and so

$$
\alpha \otimes \wedge^{2}(\alpha)=\rho^{\left(n-3,1^{3}\right)}+\rho^{(n-3,2,1)}+\rho^{\left(n-2,1^{2}\right)}+\rho^{(n-2,2)}+\rho^{(n-1,1)} .
$$

Similarly,

$$
\alpha \otimes \rho^{(n-2,2)}=\rho^{(n-3,3)}+\rho^{(n-3,2,1)}+\rho^{\left(n-2,1^{2}\right)}+\rho^{(n-2,2)}+\rho^{(n-1,1)} .
$$

It now follows that

$$
\begin{aligned}
\rho^{(n-3,2,1)} & =\alpha \otimes \wedge^{2}(\alpha)-\wedge^{3}(\alpha)-\alpha \otimes \alpha+1, \\
\rho^{(n-3,3)} & =\alpha \otimes \rho^{(n-2,2)}-\alpha \otimes \wedge^{2}(\alpha)+\wedge^{3}(\alpha),
\end{aligned}
$$

and we arrive at the above formulae for $\rho^{\lambda}(g)$.

Next assume that $g$ has exactly $k_{i}$ cycles of length $i, i=1,2, \ldots$, in its decomposition into disjoint cycles. We will write $g=\left(1^{k_{1}} 2^{k_{2}} \ldots\right)$ in this case. Then $\alpha(g)=k_{1}-1$, $\alpha\left(g^{2}\right)=k_{1}+2 k_{2}-1$, and $\alpha\left(g^{3}\right)=k_{1}+3 k_{3}-1$. Let $\rho=\rho^{\lambda}$ for short. We also assume that $g$ is not 1 or a 2 -cycle; in particular, $-1 \leq \alpha(g) \leq n-4$ and $-1 \leq \alpha\left(g^{2}\right) \leq n-1$.

Consider the case $\lambda=\left(n-2,1^{2}\right)$. Then $\rho(\mathbf{t})=\left(n^{2}-7 n+10\right) / 2$, and

$$
1-n \leq-\alpha\left(g^{2}\right) \leq 2 \rho(g)=\alpha(g)^{2}-\alpha\left(g^{2}\right) \leq(n-4)^{2}+1 \leq 2 \rho(\mathbf{t}) .
$$


Next assume that $\lambda=(n-2,2)$. Then $\rho(\mathbf{t})=\left(n^{2}-7 n+12\right) / 2$. Furthermore,

$$
-1-2 n \leq 2 \rho(g)=\alpha(g)(\alpha(g)-2)+\alpha\left(g^{2}\right)-2 \leq(n-4)(n-6)+n-3 \leq 2 \rho(\mathbf{t}) .
$$

Now we consider the case $\lambda=(n-3,3)$. Then $\rho(\mathbf{t})=(n-3)(n-4)(n-5) / 6$, and

$$
\rho(g)=\left(k_{1}-1\right)\left(k_{1}-2\right)\left(k_{1}-3\right) / 6+\left(k_{1}-1\right)\left(k_{2}-1\right)+k_{3} .
$$

The desired estimate is clear if $k_{1}=0$. Assume that $k_{1}, k_{2} \geq 1$, in particular $\rho(g) \geq 0$. Since $\rho(g)$ is increasing when we replace $\left(1^{k_{1}} 2^{k_{2}} 3^{k_{3}}\right)$ by $\left(1^{k_{1}+k_{3}} 2^{k_{2}+k_{3}}\right)$, we may assume that $k_{3}=0$. Also, since $\rho(g)$ is increasing when we replace $\left(1^{k_{1}} 2^{k_{2}}\right)$ by $\left(1^{k_{1}+2} 2^{k_{2}-1}\right)$ for $k_{2} \geq 2$, we may assume that $k_{2}=1$. It follows that $\rho(g)$ is maximized when $g$ is a 2-cycle. Finally, let $k_{2}=0$. Again the desired estimate is clear if $1 \leq k_{1} \leq 5$, so we may assume $k_{1} \geq 6$ and $k_{j} \geq 1$ for some $j \geq 3$; in particular, $\rho(g) \geq 0$. Notice that $\rho(g)$ increases when we replace a $j$-cycle by $\left(1^{j-3} 3^{1}\right)$ for $j \geq 4$, and when we replace $\left(1^{k_{1}} 3^{k_{3}}\right)$ by $\left(1^{k_{1}+3} 3^{k_{2}-1}\right)$ for $k_{3} \geq 2$. Hence $\rho(g) \leq \rho(3$-cycle $) \leq \rho(\mathbf{t})$.

Next assume that $\lambda=\left(n-3,1^{3}\right)$. Then $\rho(\mathbf{t})=(n-2)(n-3)(n-7) / 6$. The desired estimate is clear if $\alpha(g) \leq 1$ or if $n=9$. On the other hand, if $2 \leq \alpha(g) \leq n-5$ and $n \geq 10$, then

$6|\rho(g)|=\left|\alpha(g)^{3}-3 \alpha(g) \alpha\left(g^{2}\right)+2 \alpha\left(g^{3}\right)\right| \leq(n-5)^{3}+3(n-5)+2(n-1) \leq 6 \rho(\mathbf{t})$.

Also, if $\alpha(g)=n-4$ and $n \geq 10$, then $6 \rho(g)=(n-4)^{2}(n-7)+2(n-1)<6 \rho(\mathbf{t})$.

Finally, we consider the case $\lambda=(n-3,2,1)$. Then $\rho(\mathbf{t})=(n-2)(n-4)(n-6) / 3$, and

$$
3 \rho(g)=\left(k_{1}-1\right)^{3}-3\left(k_{1}-1\right)^{2}-\left(k_{1}-1\right)-3\left(k_{3}-1\right) .
$$

The desired estimate is clear if $k_{1} \leq 4$, so we may assume $k_{1} \geq 5$. Observe that $\rho(g)$ increases when we replace a $j$-cycle by $\left(1^{j-2} 2^{1}\right)$ for $j \geq 4$, or if we replace a 3 -cycle by $\left(1^{1} 2^{1}\right)$ for $k_{3} \geq 1$, or if we replace a 2 -cycle by $\left(1^{2}\right)$ for $k_{2} \geq 2$. It now readily follows that $|\rho(g)| \leq \rho(\mathbf{t})$.

Proposition 3.4. Let $G$ be as in (\$) and $S=\mathrm{A}_{n}$ for some $n \geq 8$. Then $\chi(1)=n-1$, $L=\mathrm{A}_{n}$, and $L$ acts on $V$ as on its deleted natural permutation module. Moreover, one of the following holds.

(i) age ${ }^{*}(g)=1 / 2, \Delta(g)=2$, and a scalar multiple of $g$ is a 2-cycle, acting on $V$ as a reflection.

(ii) age $(g)=1, \Delta(g)=3$ or 4 , and a scalar multiple of $g$ is a 3-cycle, or a double transposition, both acting on $V$ as a (complex) bireflection.

Proof. 1) First we consider the case $L=2 \mathrm{~A}_{n}$. Since $\operatorname{Aut}\left(\mathrm{A}_{n}\right)=\mathrm{S}_{n}$ and $C_{G}(L / Z(L))=$ $Z(G)$, we may replace $G$ by $H \in\left\{2 \mathrm{~A}_{n}, 2 \mathrm{~S}_{n}\right\}$. By Lemma 2.23 and (4) we have $4.556>$ $\Delta(g) \geq \chi(1) / 8$ and so $\chi(1) \leq 36$. It is well known (cf. e.g. [KT]) that $\chi(1) \geq 2^{\lfloor n / 2\rfloor-1}$, hence $n \leq 13$. Now we can go through the irreducible spin characters of $H$ for $8 \leq$ $n \leq 13$ as listed in [Atlas] and check that $\Delta(g)$ can be less than 4.556 only when $\chi(1)=8, n=8$ or 9 , and $\Delta(g)=4$. However, in this exceptional case, age* $(g)>1$. 
2) Next we assume that $L=\mathrm{A}_{n}$ and moreover $\left.\chi\right|_{L}$ is not the character of the deleted natural permutation module. Again as above we may replace $G$ by $H \in\left\{\mathrm{A}_{n}, \mathrm{~S}_{n}\right\}$. By Proposition 2.22(ii), $|\chi(g) / \chi(1)| \leq 1-1 / 2 n$, whence $4.556>\Delta(g) \geq \chi(1) / 2 n$ and $\chi(1)<(9.112) n$. Also we choose $\lambda \vdash n$ such that $\left.\chi\right|_{L}$ is an irreducible constituent of $\left.\rho^{\lambda}\right|_{L}$. By our assumption, $\lambda \notin R_{n}(1)$.

Consider the case $n \geq 14$. Then by Lemma 3.2(i) (and by [GAP] for $n=14$ ), either $\rho^{\lambda}(1) \geq n(n-1)(n-5) / 6$, or $\lambda \in R_{n}(2)$. Since $\chi(1) \geq \rho^{\lambda}(1) / 2$, in the former case we would have $\chi(1) \geq(9.75) n$, a contradiction. Hence $\lambda \in R_{n}$ (2); in particular, $\left.\chi\right|_{L}=\left.\rho^{\lambda}\right|_{L}$. But in this case, Lemma 3.3 and its proof imply that $\Delta(g) \geq \Delta(\mathbf{t}) \geq 2 n-6 \geq 22$, again a contradiction.

Finally, let $8 \leq n \leq 13$. An inspection of irreducible characters of $H$ [Atlas] reveals that $\Delta(g)>4.556$ in all cases.

3) We have shown that $\chi(1)=n-1$ and $\left.\chi\right|_{L}$ is the character of the deleted natural permutation module. We may write $g=\alpha h$, where $h \in \mathrm{S}_{n}$ and $\alpha \in \mathbb{C}^{\times}$. Then $|\chi(g)|=$ $|\chi(h)|=|\mu(h)-1|$, where $\mu(h)$ is the number of points fixed by the permutation $h$. Since $\Delta(g)<4.556$ and $n \geq 8$, we see that $n-2 \geq \mu(h) \geq n-4$. If $\mu(h)=n-2$, then $h$ is a 2 -cycle, $\Delta(g)=2$ and age* $(g)=\operatorname{age}(h)=1 / 2$. If $\mu(h)=n-3$, then $h$ is a 3 -cycle, $\Delta(g)=3$ and age* $(g)=\operatorname{age}(h)=1$. If $\mu(h)=n-4$, then $\Delta(g)=4$ and $h$ is either a double transposition, or a 4-cycle. In the former case age* $(g)=\operatorname{age}(h)=1$. In the latter case $\Delta(g)=4$ and age* $(g)>1$ by Lemma 2.21(iii).

From now on we may assume that $S \not \mathrm{A}_{n}$ for any $n \geq 8$. By Lemma 2.19(i) and (4), there is some $h \in L \backslash Z(L)$ with

$$
\Delta(h) \leq 4 \Delta(g)<18.224
$$

which implies by Proposition 2.22(i) that $\chi(1) / 20<18.224$ and so

$$
\chi(1) \leq 364
$$

Let $\mathfrak{d}(S)$ denote the smallest degree of a projective complex irreducible representation of $S$. We will freely use the precise value of $\mathfrak{d}(S)$ as recorded in [T].

\subsection{Classical groups}

To handle the finite classical groups, we will also need to estimate character ratios for their Weil representations (cf. [TZ2], [GMST] and references therein for definitions and detailed information on Weil representations).

Lemma 3.5. Let $\chi$ be an irreducible complex Weil character of $L=S L_{n}(q)$ or $S U_{n}(q)$, $n \geq 3,(n, q) \neq(3,2),(3,3),(4,2)$, and let $g \in L \backslash Z(L)$. Then

$$
\frac{|\chi(g)|}{\chi(1)}<\frac{q^{n-1}+q^{2}}{q^{n}-q} \leq \frac{2}{3} .
$$


Proof. First we consider the case $L=S U_{n}(q)$ and let $\mathcal{N}=\mathbb{F}_{q^{2}}^{n}$ denote the natural module for $L$. Fix a primitive $(q+1)^{\text {th }}$ root $\delta$ of unity in $\mathbb{F}_{q^{2}}$, and let $d_{k}$ denote the dimension of the subspace $\operatorname{Ker}\left(g-\delta^{k} \cdot\right.$ Id) of $\mathcal{N}$, for $0 \leq k \leq q$. Then the explicit formula for $\chi$ as given in [TZ2] implies that $\chi(1) \geq\left(q^{n}-q\right) /(q+1)$ and $(q+1)|\chi(g)| \leq S:=$ $\sum_{k=0}^{q} q^{d_{k}}$. Clearly, $\sum_{k=0}^{q} d_{k} \leq n$ and $0 \leq d_{k} \leq n-1$. Without loss we may assume that $d_{1}=\max _{0 \leq k \leq q} d_{k}$. Now $S \leq(q+1) q^{n-3}<q^{n-1}$ if $d_{1} \leq n-3, S \leq q^{n-2}+q^{2}+q-1<$ $q^{n-1}+q^{2}$ if $d_{1}=n-2$, and $S \leq q^{n-1}+2 q-1<q^{n-1}+q^{2}$ if $\bar{d}_{1}=n-1$, and so we are done.

Next, let $L=S L_{n}(q)$ and let $\mathcal{N}=\mathbb{F}_{q}^{n}$ denote the natural module for $L$. Fix a primitive $(q-1)^{\text {th }}$ root $\epsilon$ of unity in $\mathbb{F}_{q}$, and let $e_{k}$ denote the dimension of the subspace $\operatorname{Ker}(g-$ $\epsilon^{k}$. Id) of $\mathcal{N}$, for $0 \leq k \leq q-2$. Then the explicit formula for $\chi$ as given in [TZ2] implies that $\chi(1) \geq\left(q^{n}-q\right) /(q-1)$ and $(q-1)|\chi(g)| \leq R:=\sum_{k=0}^{q-2} q^{d_{k}}+2 q-2$. Clearly, $\sum_{k=0}^{q-2} e_{k} \leq n$ and $0 \leq e_{k} \leq n-1$. Without loss we may assume that $e_{1}=\max _{0 \leq k \leq q} e_{k}$. Now $R \leq(q-1)\left(q^{n-3}+2\right)<q^{n-1}+q^{2}$ if $e_{1} \leq n-3, R \leq q^{n-2}+q^{2}+3 q-5<q^{n-1}+q^{2}$ if $e_{1}=n-2$, and $R \leq q^{n-1}+4 q-5<q^{n-1}+q^{2}$ if $e_{1}=n-1$, and so we are again done.

Lemma 3.6. Let $\chi$ be an irreducible complex Weil character of $L=S p_{2 n}(q)$, $q$ odd, $n \geq 2$, and let $g \in L \backslash Z(L)$. Then

$$
\frac{|\chi(g)|}{\chi(1)} \leq \begin{cases}\frac{q^{n-1 / 2}+1}{q^{n}+1}, & q \equiv 1(\bmod 4) \text { and } \pm g \text { is a transvection, } \\ \frac{\left(q^{2 n-1}+1\right)^{1 / 2}}{q^{n}-1}, & q \equiv 3(\bmod 4) \text { and } \pm g \text { is a transvection, } \\ \frac{2 q^{n-1}}{q^{n}-1}, & \pm g \text { is not a transvection. }\end{cases}
$$

In particular, $|\chi(g) / \chi(1)| \leq 0.675$ unless $(n, q)=(3,3),(2,3)$.

Proof. Note that $\chi(1)=\left(q^{n}-\epsilon\right) / 2$ for some $\epsilon= \pm 1$. If $\pm g$ is a transvection in $L$ then by [TZ2], $|\chi(g)|=\left(q^{n-1 / 2}-\epsilon\right) / 2$ when $q \equiv 1(\bmod 4)$, and $|\chi(g)|=\sqrt{q^{2 n-1}+1} / 2$ when $q \equiv 3(\bmod 4)$. Assume $\pm g$ is not a transvection, i.e. the subspace $\operatorname{Ker}(g \pm \operatorname{Id})$ on the natural module $\mathbb{F}_{q}^{2 n}$ of $L$ has dimension at most $2 n-2$. Also consider the reducible Weil character $\omega$ of $L$ (that has $\chi$ as one of its irreducible constituents) (cf. [GMST]). This character arises from the action of $L$ as an outer automorphism subgroup of the extraspecial $p$-group of order $p^{1+2 n f}$ and exponent $p$, where $q=p^{f}$ and $p$ is prime. By [GT3, Proposition 2.8], $|\omega(g)|,|\omega(-g)| \leq q^{n-1}$. One can write $\omega=\chi+\eta$ for another irreducible Weil character $\eta$ of $L$, and moreover $|\omega(-g)|=|\chi(g)-\eta(g)|$. It follows that $|\chi(g)| \leq q^{n-1}$.

3.3.1. $S=P S L_{n}(q), n \geq 3,(n, q) \neq(3, q \leq 7),(4,3),(5,2)$. Under these assumptions, $\mathfrak{d}(S)=\left(q^{n}-q\right) /(q-1)$. Hence (6) implies that $3 \leq n \leq 8$; moreover, $q=2$ if $n=7,8$, $q \leq 3$ if $n=6, q \leq 4$ if $n=5, q \leq 5$ if $n=4$, and $q \leq 17$ if $n=3$. In fact, if in addition $\left.\chi\right|_{L}$ is a Weil representation, then $18.224>\Delta(h) \geq \chi(1) / 3$ by Lemma 3.5, 
and so instead of (6) we have the much stronger upper bound $\chi(1) \leq 54$. Now in the cases $(n, q)=(8,2),(7,2),(6,3),(5,4),(5,3)$, and $(3, q \geq 8)$, the upper bound $(6)$ and [TZ1, Theorem 3.1] imply that $\left.\chi\right|_{L}$ is indeed a Weil representation, of degree at least 72, giving a contradiction. Also, the case $(n, q)=(4,2)$ has already been considered in Proposition 3.4.

Assume $(n, q)=(6,2)$ or $(4,4)$. Then $L=S L_{n}(q)$, and its character table is available in [GAP]. It is straightforward to check that there is no non-trivial $\chi \in \operatorname{Irr}(L)$ and $h \in$ $L \backslash Z(L)$ with $\Delta(h)<18.224$ (notice that we need to check only the non-Weil characters of degree at most 364).

It remains to analyze the case $S=P S L_{4}(5)$. The character degrees of $S L_{4}(5)$ are listed by F. Lübeck [Lu2]. In particular, we see that all the non-trivial irreducible characters of $R:=S L_{4}(5)$ have degree 155, 156 (and they are Weil characters in these two cases), 248 (and there are exactly two characters of this degree), or at least 403 . Hence we may assume that $\chi(1)=248$. An inspection of character degrees as listed in [St] shows that $G L_{4}(5)$ has no irreducible characters of degree 248. Thus $\left.\chi\right|_{R}$ is not stable under $G L_{4}(5)$. Since $\operatorname{Out}(R)$ is a dihedral group of order 8 and $G L_{4}(5)$ induces the unique cyclic subgroup of order 4 of $\operatorname{Out}(R)$, it follows that the inertia group of $\left.\chi\right|_{R}$ in $\operatorname{Out}(R)$ is an elementary abelian 2-group. But $\left.\chi\right|_{R}$ extends to $G$. Thus $G$ can induce only an elementary abelian 2-subgroup of $\operatorname{Out}(R)$. We conclude that $g^{2} \in Z(G) L$. Notice that $\chi(1)-|\chi(v)| \geq$ $\chi(1) / 20=12.4$ for any $v \in L \backslash Z(L)$ by Proposition 2.22(i). Together with (5), this implies that $4 \Delta(g) \geq \Delta(h) \geq 12.4$ and so $\Delta(g) \geq 3.1$. We will complete the case $S=P L_{4}(5)$ by showing that age* $(g)>1$.

First we suppose that $g^{2} \notin Z(G)$. Then $\Delta\left(g^{2}\right) \geq 12.4$ as above, and so age* $\left(g^{2}\right) \geq$ $24.8 /(2.9 \pi)>2.72$ by Proposition 2.17(iii). It now follows from Lemma 2.2(v) that age* $(g)>\operatorname{age}\left(g^{2}\right) / 2>1.36$. Finally, assume that $g^{2} \in Z(G)$. Then $g$ acts on a suitable basis of $V$ via the matrix $\alpha \cdot \operatorname{diag}(\underbrace{1, \ldots, 1}_{k}, \underbrace{-1, \ldots,-1}_{l})$ for some $\alpha \in \mathbb{C}^{\times}$and $1 \leq k, l<$ $k+l=248$. It is shown in [GS] that $\boldsymbol{\alpha}(g Z(G)) \leq 6$, whence $k, l \geq 42$ by Lemma 2.16 . It follows that $|\chi(g)|=|248-2 l| \leq 164, \Delta(g) \geq 84$, and age* $(g) \geq 168 /(2.9 \pi)>18$ again by Proposition 2.17(iii).

3.3.2. $S=P S U_{n}(q), n \geq 3,(n, q) \neq(3, q \leq 8),(4,2), \quad(4,3), \quad(5,2)$, $(6,2)$. Under these assumptions, $\mathfrak{d}(S)=\left(q^{n}-q\right) /(q+1)$ if $n$ is odd and $\left(q^{n}-1\right) /(q+1)$ if $2 \mid n$. Hence (6) implies that $3 \leq n \leq 10$; moreover, $q=2$ if $7 \leq n \leq 10, q \leq 3$ if $n=6, q \leq 4$ if $n=5, q \leq 7$ if $n=4$, and $q \leq 19$ if $n=3$. As in $\S 3.3 .1$, if in addition $\left.\chi\right|_{L}$ is a Weil representation, then instead of $(6)$ we have the much stronger upper bound $\chi(1) \leq 54$ (in fact $\chi(1) \leq 39$ if $(n, q)=(7,2)$ or $(4,4))$. Now in the cases $(n, q)=(10,2),(9,2)$, $(8,2),(6,3),(5,4),(5,3),(4,7)$, and $(3, q \geq 9)$, the upper bound (6) and [TZ1, Theorem 4.1] imply that $\left.\chi\right|_{L}$ is indeed a Weil representation, of degree at least 60, giving a contradiction. The same argument applies to $(n, q)=(7,2)$ as the Weil representations of $S U_{7}(2)$ have degree at least 42 and the non-Weil representations have degree at least 860 .

Assume $(n, q)=(4,4)$. Then $L=S U_{4}(4)$, and its character table is available in [GAP]. It is straightforward to check that there is no non-trivial $\chi \in \operatorname{Irr}(L)$ and 
$h \in L \backslash Z(L)$ with $\Delta(h)<18.224$ (notice that we need to check only the non-Weil characters of degree at most 364).

It remains to analyze the case $S=P S U_{4}(5)$. The character degrees of $S U_{4}(5)$ are listed by F. Lübeck [Lu2]. In particular, we see that all the non-trivial irreducible characters of $S U_{4}(5)$ have degree 104, 105 (and they are Weil characters in these two cases), 273 (and there are exactly two characters of this degree), or at least 378. Hence we may assume that $\chi(1)=273$. Checking the character table of $P S U_{4}(5)$ (available in [GAP]), we see that it also has exactly two irreducible characters of degree 273. It follows that $L=S=\mathrm{PSU}_{4}(5)$. Direct inspection of these two characters of $S$ reveals that $\Delta(h) \geq 250$, a contradiction.

3.3.3. $S=P S p_{2 n}(q), n \geq 2,(n, q) \neq(2, q \leq 5),(3,2),(3,3),(4,2)$. Under these assumptions, $\mathfrak{d}(S)=\left(q^{n}-1\right) / 2$ if $q$ is odd and $\left(q^{n}-1\right)\left(q^{n}-q\right) / 2(q+1)$ if $2 \mid q$. Hence (6) implies that $2 \leq n \leq 6$; moreover, $q=3$ if $n=6, q \leq 3$ if $n=5, q=3$, 5 if $n=4$, $q=5,7,9$ if $n=3$; if $n=2$ then either $q \leq 27$ and $q$ odd or $q=8$. Moreover, if in addition $q$ is odd and $\left.\chi\right|_{L}$ is a Weil representation, then, by Lemma 3.6, instead of (6) we have the much stronger upper bound $\chi(1) \leq 56$ (in fact $\chi(1) \leq 29$ if $(n, q)=(2,7)$ or $(2,9)$ ). Now in the cases $(n, q)=(6,3),(5,3),(4,5),(3,5),(3,7),(3,9)$, and $(2, q \geq 11)$, the upper bound (6) and [TZ1, Theorem 5.2] imply that $\left.\chi\right|_{L}$ is indeed a Weil representation, of degree at least 60 , giving a contradiction.

Assume $(n, q)=(2,7)$ or $(2,9)$. The character table of $\operatorname{Sp}_{2 n}(q)$ is determined in [Sr]. It is now straightforward to check that $\Delta(h) \geq 100$ if $\left.\chi\right|_{L}$ is a non-Weil character of degree at most 364. Moreover, the Weil characters of $S p_{4}(9)$ have degree 40 or 41 , larger than the bound 29 mentioned above. On the other hand, when $(n, q)=(2,7)$, none of the Weil characters (of degree 24 or 25) is fixed by an outer automorphism of $S p_{4}(7)$. This implies that $G=Z(G) L$ and so we may assume $g \in L$ in this case. Hence, if $\left.\chi\right|_{L}$ is a Weil character, then $4.556>\Delta(g) \geq(1-0.675) \chi(1)$ by Lemma 3.6, and so $\chi(1) \leq 14$, a contradiction. The same argument excludes the Weil characters of $S p_{8}(3)$; all other nontrivial irreducible characters of $S p_{8}(3)$ have degree at least 780 by [TZ1, Theorem 5.2], hence we are done in the case $(n, q)=(4,3)$. If $(n, q)=(5,2)$, then $\operatorname{Out}(L)=1$ and so we may assume that $g \in L$, whence $\Delta(g) \geq \chi(1) / 20 \geq 7.75$ as $\mathfrak{d}(S)=155$. Finally, inspecting the character table of $S p_{4}(8)$ (available in [GAP]), we see that $\Delta(h) \geq 168$, again a contradiction when $(n, q)=(2,8)$.

3.3.4. $S=P \Omega_{n}^{\epsilon}(q), n \geq 7,(n, q) \neq(7,3),(8,2),(10,2)$. If $(n, q) \neq(8,3)$ in addition, then $\mathfrak{d}(S) \geq 620$ by [TZ1], and so we are done. Consider the case $S=P \Omega_{8}^{ \pm}(3)$. Notice that $\operatorname{Spin}_{7}(3)$ embeds in $\operatorname{Spin}_{8}^{ \pm}(3)$ and any faithful irreducible character of $\operatorname{Spin}_{7}(3)$ has degree at least 520. Hence the bound (6) implies that $L=S$ (this can also be deduced using the list of character degrees of $\operatorname{Spin}_{8}^{ \pm}(q)$ as given in [Lu2]). Inspecting the character table of $P \Omega_{8}^{ \pm}$(3) (available in [Atlas]), we see that $\Delta(h) \geq 189$, a contradiction.

3.3.5. $S=P S L_{2}(q), q \geq 37$. In these cases, $\chi(1) \geq(q-1) / \operatorname{gcd}(2, q-1)$ and $|\chi(h)| \leq$ $(\sqrt{q}+1) / 2$ (cf. [D]). In particular, $|\chi(h) / \chi(1)| \leq 1 /(\sqrt{q}-1)<0.2$, and so (5) implies that $\chi(1) \leq 22$. Since we are assuming $q \geq 37$, this in turns forces that $q=37,41$, 
or 43 , and $\left.\chi\right|_{L}$ is in fact a Weil character. But for these values of $q$, none of the Weil characters of $L$ is fixed by an outer automorphism of $L$. Hence $G=Z(G) L$, and so we may assume that $g \in L$. Thus $|\chi(g) / \chi(1)|<0.2$ as above, and $\Delta(g)>(0.8) \chi(1) \geq$ 14.4 , a contradiction.

\subsection{Exceptional groups of Lie type}

Let $S$ be a simple exceptional group of Lie type. If $S$ is not isomorphic to ${ }^{2} B_{2}(q)$ with $q \leq 32, G_{2}(q)$ with $q \leq 7,{ }^{3} D_{4}(q)$ with $q \leq 3,{ }^{2} F_{4}(2){ }^{\prime}$, or $F_{4}(2)$, then $\mathfrak{d}(S) \geq 504$ (see e.g. [Lu1]). Consider the case $S=G_{2}(7)$. Then $L$ has a unique non-trivial irreducible character of degree at most 364 (namely 344), and this character is labeled as $\chi_{32}$ in the generic character table of $G_{2}(q)[\mathrm{H}]$. One can now check that $\Delta(h) \geq 332$ for $\left.\chi\right|_{L}=\chi_{32}$. Similarly, if $S={ }^{3} D_{4}(3)$ then $L$ has a unique non-trivial irreducible character of degree at most 364 (namely 219). This character is unipotent, and its values are computed in [Sp]. In particular, one can check that $\Delta(h) \geq 195$ in this case.

\subsection{Small groups}

The list of our "small" groups consists of all the finite simple groups not considered in the above subsections, that is: $\mathrm{A}_{n}$ with $5 \leq n \leq 7, \operatorname{PSL}_{2}(q)$ with $7 \leq q \leq 32, \operatorname{PSL}_{3}(q)$ with $3 \leq q \leq 7, P_{4} L_{4}(3), S L_{5}(2), P S U_{3}(q)$ with $3 \leq q \leq 8, S U_{4}(2), P S U_{4}(3), S U_{5}(2)$, $P S U_{6}(2), \operatorname{Sp}_{4}(4), P S p_{4}(5), S_{6}(2), P S p_{6}(3), S p_{8}(2), \Omega_{7}(3), \Omega_{8}^{ \pm}(2), \Omega_{10}^{ \pm}(2),{ }^{2} B_{2}(q)$ with $8 \leq q \leq 32, G_{2}(q)$ with $3 \leq q \leq 5,{ }^{3} D_{4}(2),{ }^{2} F_{4}(2){ }^{\prime}, F_{4}(2)$, and 26 sporadic simple groups. Notice that the character table of the universal cover of $S$ is known (see [GAP]) in all these cases.

Recall we are assuming that $L=G^{(\infty)}$ is quasi-simple, and $\chi \in \operatorname{Irr}(G)$ is irreducible over $L$; moreover, $1<\chi(1) \leq 364$ by (6). The last condition excludes the cases $S \in$ $\left\{J_{4}, F i_{23}, F i_{24}^{\prime}, L y, B M=F_{2}, M=F_{1}\right\}$. We will use the character tables of the universal cover of $S$ as given in [Atlas], as well as the notation therein for the conjugacy classes in $G / Z(G)$.

\subsubsection{Sporadic groups. One can check that}

- $\Delta(g) \geq 6$ if $S=M_{22}$, Suz;

- $\Delta(g) \geq 8$ if $S=M_{11}, M_{12}$, or if $S=J_{2}$ but $\chi(1)>6$; and

- $\Delta(g) \geq 12$ if $S=M_{23}, M_{24}, J_{1}, J_{3}, H S, M c L, H e, R u, H N, F_{22}, C o_{3}, C o_{2}, C o_{1}, O^{\prime} N$, Th,

for all $\chi$ satisfying the above hypotheses.

Assume that $S=J_{2}$ and $\chi(1)=6$; in particular, $L=2 \cdot J_{2}$ and $G=Z(G) L$. Then one can check that $\Delta(g) \geq 5-\sqrt{5}>8-4 \sqrt{2}$. Next, suppose that $0<$ age $^{*}(g) \leq 1$; in particular, $|\chi(g)|>1.444$. Then, in the notation of [Atlas], we may assume that $\left.\chi\right|_{L}=$ $\chi_{22}$, and the class of $g Z(G)$ in $S$ is one of the following: $2 A, 3 A, 4 A, 5 B, 5 C, 10 D$, and $15 B$. The first two cases lead to the row of $2 \cdot J_{2}$ in Table I. In the last two cases, in the notation of Corollary 2.18 we have $\delta \geq 6 \pi / 5$, but $\Delta(g)>4.38$, whence age* $(g)>1$ 
by Corollary 2.18. In the case of class $4 A$, a multiple of $g$ has spectrum $1,1, i, i,-i,-i$, and so age* $(g)>1$ by Lemma 2.21 (with $i=\sqrt{-1}$ ). Finally, in the case of classes $5 B$ and $5 C$, none of the eigenvalues of $g$ occurs with multiplicity $\geq 3$, and so age* $(g)>1$ by Lemma 2.21 .

3.5.2. Small alternating groups: $S=\mathrm{A}_{n}$ with $5 \leq n \leq 7$. Arguing as in the proof of Proposition 3.4 (and using Lemmas 2.20 and 2.21), we may assume that $\left.\chi\right|_{L}$ is not the character of the deleted natural permutation module. First we consider the case $S=$ A5. Direct check using [Atlas] shows that $\Delta(g) \geq(3-\sqrt{5}) / 2$ if $d=2$ and $\Delta(g) \geq$ $(5-\sqrt{5}) / 2>3-\sqrt{3}$ if $d=3$. Assume $d=4$ (and so $L=2 \cdot \mathrm{A}_{5}$ by our assumptions). If $g Z(G)$ belongs to the class $5 A$ or $5 B$ of $G / Z(G)$, then $\Delta(g)=3$ and age* $(g)>1$ by Lemma 2.21(iv). If $g Z(G)$ belongs to the class $4 A$, then $\Delta(g)=4$ and age* $(g)>1$ by Lemma 2.20 (with $m=2$ ). Similar arguments apply to the case $d=5$. If $d=6$, then $\Delta(g) \geq 6-\sqrt{2}>4.556$.

Assume $n=6$. Then the assumptions on $\chi, L$, and $\Delta(g)$ lead to one of the following three possibilities.

- $d=3, L=3 \mathrm{~A}_{6}$, and $\Delta(g) \geq(5-\sqrt{5}) / 2>3-\sqrt{3}$.

- $d=4, L=2 \cdot \mathrm{A}_{6}$, and $\Delta(g) \geq 2$. The classes $2 A, 2 B, 2 C, 3 A, 3 B$, and $6 B$ lead to three rows of Table I. The other classes are excluded by Lemmas 2.20 and 2.21.

- $d=6, L=3 \cdot \mathrm{A}_{6}, \Delta(g)=4$, and $g Z(G)$ belongs to the class $2 A$, which leads to a row in Table I.

Assume $S=\mathrm{A}_{7}$. Then the assumptions on $\chi, L$, and $\Delta(g)$ lead to one of the following two possibilities.

- $d=4, L=2 \cdot \mathrm{A}_{7}, G=Z(G) L$, and $\Delta(g) \geq 2$. The classes $2 A, 3 A, 3 B$, and $7 A$ lead to two rows of Table I. The other classes are excluded by Lemmas 2.20 and 2.21.

- $d=6, L=3 \cdot \mathrm{A}_{7}, G=Z(G) L, \Delta(g)=4$, and $g Z(G)$ belongs to the classes $2 A$ or $6 A$. The former case leads to a row in Table I, and age* $(g)>1$ in the latter case by Lemma 2.20 (with $m=2$ ).

Lemma 3.7. There are subgroups $G=C_{3} \times 2 \mathrm{~A}_{m}<G L_{4}(\mathbb{C})$ with $m=6,7$ which give a basic non-RT pair not of reflection type. This pair is of $A V$-type if $m=6$.

Proof. The faithful representation of $G$ on $V=\mathbb{C}^{4}$ gives rise to a unique conjugacy class $g^{G}$ of non-central elements of age $<1$, namely class $3 A$ in $G / Z(G) \simeq \mathrm{A}_{m}$. Let $K:=\left\langle g^{G}\right\rangle$. Then $Z(G) K=G$ by simplicity of $G / Z(G)$, but $g \notin[G, G]=2 \mathrm{~A}_{m}$. It follows that $K=G$ and so $G$ gives a basic non-RT pair. Furthermore, $Z(G L(V)) G$ does not contain any complex reflection, hence this pair is not of reflection type. Finally, if $m=6$ then the representation of $G=C_{3} \times S L_{2}(9)<C_{3} \times S p_{4}$ (3) on $V$ can be written over $\mathbb{Q}(\sqrt{-3})$ and so the corresponding basic non-RT pair is of AV-type.

3.5.3. Small finite groups of Lie type. Let $S$ be any of the small simple finite groups of Lie type listed at the beginning of $\S 3.5$. Using [Atlas], it is straightforward to check that $\Delta(g) \geq 5$ for all characters $\chi$ satisfying the above hypotheses, except possibly for one 
of the following cases. (Note that it suffices to consider only subgroups of $G$ that induce cyclic extensions of $S$ in $\operatorname{Aut}(S)$, since $\langle g, L\rangle$ is such a subgroup.)

- $d=8, L=2 \cdot \Omega_{8}^{+}(2)$. Here, either $\Delta(g)=2, g Z(G)$ belongs to class $2 F$, and $g$ acts as a reflection, or $\Delta(g) \geq 3$. In the latter case, either we get the row of $\Omega_{8}^{+}(2)$ in Table I with complex bireflections of order 2 and 3, or age* $(g)>1$ by Lemma 2.21 (when $g Z(G)$ has order $\leq 5$ ) and Corollary 2.18 (with $\delta \geq 4 \pi / 3$ ).

- $d=7$ or 8 , and $S=S p_{6}(2)$. If $d=8$, then $\Delta(g) \geq 4$ and age* $(g)>1$. Assume $d=7$. Then either $\Delta(g)=2, g Z(G)$ belongs to class $2 A$, and $-g$ acts as a reflection, or $\Delta(g) \geq 3$. In the latter case, either we get the row of $S p_{6}(2)$ in Table I with complex bireflections of order 2 and 3, or age* $(g)>1$ by Lemma 2.21 .

- $d=10, L=S U_{5}(2), \Delta(g)=4, g Z(G)$ belongs to class $2 A$, and $g$ acts as a bireflection.

- $d=6,20$, or 21 , and $S=P S U_{4}(3)$. Assume $d=6$. Then either $\Delta(g)=2, g Z(G)$ belongs to class $2 D$, and $g$ acts as a reflection, or $\Delta(g) \geq 3$. In the latter case, either we get the row of $\mathrm{PSU}_{4}(3)$ in Table I with complex bireflections of order 2 and 3 and an element with spectrum $\left(1,1,1, e^{2 \pi / 3}, e^{2 \pi / 3}, e^{2 \pi / 3}\right)$, or age* $(g)>1$ by Lemma 2.21 and Corollary 2.18 (with $\delta \geq 4 \pi / 3$ ) (and a direct check for some elements of order 6). If $d=21$ and $L=S$, then $\Delta(g) \geq 12$. In all the remaining cases, $\Delta(h) \geq 13$ for all $h \in L \backslash Z(G)$, and so $\Delta(g) \geq 13 / 4$ by Lemma 2.19 . We claim that we also have age* $(g)>1$. Assume the contrary: age* $(g) \leq 1$. Let $K$ be any subgroup of $G$ that contains $L$ and induces a subgroup $C_{2}$ of $\operatorname{Out}(S)=D_{8}$ while acting on $L$. It is straightforward to check that, for any $h \in K \backslash Z(K), \Delta(h) \geq 10$ and so age* $(h)>2.19$ by Corollary 2.18 . Notice that $\operatorname{Out}(S)=D_{8}$ has exponent 4. Hence, if $g^{2} \notin Z(G)$, we have $g^{2} \in K \backslash Z(K)$ for a subgroup $K$ of the aforementioned type, and so age* $\left(g^{2}\right)>2.19$ and age* $(g)>1.095$ by Lemma 2.2(v). Thus $g^{2} \in Z(G)$, and so modulo scalars we may assume that $g$ has two eigenvalues 1 , resp. -1 , with multiplicity $m$, resp. $d-m$. By [GS], $\alpha(g) \leq 6$ and so $m, d-m \leq d-4$ by Lemma 2.16. It follows that $|\chi(g)|=|2 m-d| \leq d-8$, whence $\Delta(g) \geq 8$ and age* $(g)>1$.

- $d=4,5$, or 6 , and $S=S U_{4}(2) \simeq P S p_{4}(3)$. Assume $d=6$. Then either $\Delta(g)=2$, $g Z(G)$ belongs to class $2 C$, and $g$ acts as a reflection, or $\Delta(g) \geq 3$. In the latter case, either we get a row with $(d, L)=\left(6, S U_{4}(2)\right)$ in Table I, or age* $(g)>1$ by Lemma 2.21 and Corollary 2.18 (with $\delta \geq 4 \pi / 3$ ). Assume $d=5$. Then either $\Delta(g)=2, g Z(G)$ belongs to class $2 A$, and $-g$ acts as a reflection, or $\Delta(g) \geq 5-\sqrt{7}>8-4 \sqrt{2}$. In the latter case, either we get two rows with $(d, L)=\left(5, S U_{4}(2)\right)$ in Table I, or age* $(g)>1$ by Lemma 2.21 and Corollary 2.18 (with $\delta \geq 4 \pi / 3$ ) (and a direct check for some elements of order 6). Finally, assume $d=4$, and so $L=S p_{4}(3)$. This case by far has the most (twelve) classes of elements $g$ with $0<$ age* $(g) \leq 1$ (leading to two rows in Table I), and is handled by a direct case-by-case argument. In this case we always have $\Delta(g) \geq 4-\sqrt{7}$.

- $d=6$ or 7 , and $S=S U_{3}(3)$. Here we have $\Delta(g) \geq 3$, and, aside from the entries with $(d, L)=\left(6, S U_{3}(3)\right)$ and $\left(7, S U_{3}(3)\right)$ in Table I, age* $(g)>1$ by Lemmas 2.20 , 2.21, and Corollary 2.18 (with $\delta \geq 4 \pi / 3$ ).

- $d=6$ and $L=6 \cdot P S L_{3}(4)$. Here, $\Delta(g) \geq 4$, and either we are in the row of $(d, L)=\left(6,6 \cdot P S L_{3}(4)\right)$ in Table I, or age* $(g)>1$. 
- $d=6$ or 7 , and $S=P S L_{2}$ (13). If $d=7$, then $\Delta(g)>4.69$. If $d=6$, then either $\Delta(g) \geq 5$, or $\Delta(g)>3.69$ and age* $(g)>1$ by Corollary 2.18 (with $\delta=16 \pi / 13$ ).

- $d=5$ or 6 , and $S=P S L_{2}(11)$. If $d=6$, then either $\Delta(g) \geq 5$, or $\Delta(g)>4.26$ and $\operatorname{age}^{*}(g)>1$ by Corollary 2.18 (with $\delta=14 \pi / 11$ ). If $d=5$, then $\Delta(g) \geq 5-\sqrt{3}$, and either we are in the row of $(d, L)=\left(5, P S L_{2}(11)\right)$ in Table I, or age* $(g)>1$ (by direct calculation).

- $d=3$, 4, or 6 , and $S=P S L_{2}$ (7). If $d=3$, then $\Delta(g) \geq 3-\sqrt{2}$. If $d=6$, then either we are in the row $(d, L)=\left(6, P S L_{2}(7)\right)$ of Table I, or $\Delta(g)>4$ and age* $(g)>1$ by Corollary 2.18 (with $\delta \geq 5 \pi / 4$ ). Finally, if $d=4$, then $\Delta(g) \geq 4-\sqrt{2}$, and either we arrive at the row $(d, L)=\left(4, S L_{2}(7)\right)$ of Table I, or age* $(g)>1$ (by a direct check).

We have completed the proof of Theorem 1.3, as well as of the following result which we will need later.

Theorem 3.8. Let $G<G L(V)$ be an almost quasi-simple subgroup such that the $G$ module $V$ is irreducible, primitive, and tensor indecomposable. Assume that $0<$ age* $(g)$ $\leq 1$ for some $g \in G$, and that $d:=\operatorname{dim}(V) \geq 4$. Then $\left(d, G^{(\infty)}, g, \Delta(g)\right.$, age* $\left.(g)\right)$ is as listed in Table I.

In Table I, in the cases where $0<\operatorname{age}^{*}(g)<1$, we indicate a minimal group $G$ containing $h$ with age $(h)=\operatorname{age}^{*}(g)$. We also list the conjugacy class of $g Z(G)$ in $G / Z(G)$ using the notation of [Atlas], for one representative of the Aut $(L)$-conjugacy class of $\left.\chi\right|_{L}$. The notation $L * C_{4}$ stands for a central product of $L$ by a cyclic group of order 4 .

Remark 3.9. The spectra of elements $g$ with $0<\operatorname{age}^{*}(g) \leq 1$ that occur in Table I are listed as follows:

(a1) $(-1,1, \ldots, 1)$ (a reflection, age $=1 / 2)$.

(a2) $\left(e^{2 \pi i / 3}, 1,1,1\right)($ a complex reflection, age $=1 / 3)$.

(a3) $\left(e^{4 \pi i / 3}, 1,1,1\right)($ a complex reflection, age $=2 / 3)$.

(a4) $\left(e^{2 \pi i / 3}, e^{2 \pi i / 3}, 1, \ldots, 1\right)($ age $=2 / 3)$.

(a5) $\left(e^{\pi i / 3},-1,1,1\right)($ age $=2 / 3)$.

(b1) $(-1,-1,1, \ldots, 1)$ (a bireflection, age $=1)$.

(b2) $\left(e^{2 \pi i / 3}, e^{4 \pi i / 3}, 1, \ldots, 1\right)($ a complex bireflection, age $=1)$.

(b3) $\left(e^{2 \pi i / 3}, e^{2 \pi i / 3}, e^{2 \pi i / 3}, 1, \ldots, 1\right)($ age $=1)$.

(b4) $\left(e^{\pi i / 2}, e^{3 \pi i / 2}, 1,1\right)($ a complex bireflection, age $=1)$.

(b5) $\left(e^{\pi i / 3}, e^{2 \pi i / 3},-1,1\right)($ age $=1)$.

(b6) $\left(e^{\pi i / 3}, e^{\pi i / 3}, e^{4 \pi i / 3}, 1\right)($ age $=1)$.

(b7) $\left(e^{2 \pi i / 7}, e^{4 \pi i / 7}, e^{8 \pi i / 7}, 1\right)($ age $=1)$.

(b8) $\left(e^{\pi i / 6}, e^{2 \pi i / 3}, e^{7 \pi i / 6}, 1\right)($ age $=1)$.

Additionally, the following spectra also occur for the groups of extraspecial type:

(c1) $\left(e^{\pi i / 2}, e^{\pi i / 2}, 1,1\right)($ age $=1 / 2)$.

(c2) $\left(e^{\pi i / 4}, e^{\pi i / 2}, e^{5 \pi i / 4}, 1\right)($ age $=1)$.

(c3) $\left(e^{\pi i / 4}, e^{3 \pi i / 4},-1,1\right)($ age $=1)$.

(c4) $\left(e^{2 \pi i / 5}, e^{4 \pi i / 5}, e^{4 \pi i / 5}, 1,1\right)($ age $=1)$.

(c5) $\left(e^{2 \pi i / 5}, e^{2 \pi i / 5}, e^{6 \pi i / 5}, 1,1\right)($ age $=1)$.

(c6) $\left(e^{\pi i / 2}, e^{\pi i / 2}, e^{\pi i / 2}, e^{\pi i / 2}, 1,1,1,1\right)($ age $=1)$. 
Table I. Non-central elements of small age in almost quasi-simple groups.

\begin{tabular}{|c|c|c|c|c|c|}
\hline$d$ & $L:=G^{(\infty)}$ & $G$ & $g$ & $\Delta(g)=d_{2}(g)^{2} / 2$ & $\operatorname{age}^{*}(g)$ \\
\hline 4 & $S p_{4}(3)$ & $L \times 3$ & $\left\{\begin{array}{c}3 B \\
3 A, 3 C, 6 A\end{array}\right.$ & $\left\{\begin{array}{c}4-\sqrt{7} \\
4-\sqrt{7}, 2,4-\sqrt{3}\end{array}\right.$ & $\begin{array}{l}1 / 3 \\
2 / 3\end{array}$ \\
\hline 4 & $2 \cdot A_{6}$ & $L \times 3$ & $3 A$ & 2 & $2 / 3$ \\
\hline 4 & $2 \cdot A_{7}$ & $L \times 3$ & $3 A$ & 2 & $2 / 3$ \\
\hline 4 & $2 \cdot A_{5}$ & $L$ or $L * C_{4}$ & $2 A$, resp. $3 A$ & 4, resp. 3 & 1 \\
\hline 4 & $2 \cdot A_{5}$ & $L \cdot 2$ & $2 B$, resp. $6 A$ & 4 , resp. $4-\sqrt{3}$ & 1 \\
\hline 4 & $S L_{2}(7)$ & $L$ or $L * C_{4}$ & $2 A$, resp. $3 A, 7 B$ & 4, resp. $3,4-\sqrt{2}$ & 1 \\
\hline 4 & $2 \cdot A_{6}$ & $L$ or $L * C_{4}$ & $2 A$, resp. $3 B$ & 4, resp. 3 & 1 \\
\hline 4 & $2 \cdot A_{6}$ & $L \cdot 2$ & $2 B$, resp. $2 C, 6 B$ & 4, resp. $4,4-\sqrt{3}$ & 1 \\
\hline 4 & $2 \cdot A_{7}$ & $L$ or $L * C_{4}$ & $2 A$, resp. $3 B, 7 A$ & 4 , resp. 3,2 & 1 \\
\hline 4 & $\mathrm{Sp}_{4}(3)$ & $L$ or $L * C_{4}$ & $\left\{\begin{array}{c}2 A B, 3 A, 3 D \\
4 A, 6 B E, 12 A\end{array}\right.$ & $\left\{\begin{array}{l}4,4-\sqrt{7}, 3 \\
2,4-\sqrt{3}, 3\end{array}\right.$ & 1 \\
\hline 5 & $S U_{4}(2)$ & $L \times 2$ & $2 A$ & 2 & $1 / 2$ \\
\hline 5 & $S U_{4}(2)$ & $L \times 3$ & $3 A$ & $5-\sqrt{7}$ & $2 / 3$ \\
\hline 5 & $\mathrm{~A}_{5}$ & $L$ & $2 A$ & 4 & 1 \\
\hline 5 & $A_{6}$ & $S_{6} \times 2$ & $(12)(34)(56)$ & 4 & 1 \\
\hline 5 & $P S L_{2}(11)$ & $L$ & $2 A$ & 4 & 1 \\
\hline 5 & $S U_{4}(2)$ & $L$ & $2 B$, resp. $3 D$ & 4, resp. 3 & 1 \\
\hline 6 & $S U_{4}(2)$ & $L \cdot 2$ & $2 C$ & 2 & $1 / 2$ \\
\hline 6 & $6_{1} \cdot P S U_{4}(3)$ & $L \cdot 2_{2}$ & $2 D$ & 2 & $1 / 2$ \\
\hline 6 & $P S L_{2}(7)$ & $L$ & $2 A$ & 4 & 1 \\
\hline 6 & $3 \cdot A_{6}$ & $L$ & $2 A$ & 4 & 1 \\
\hline 6 & $3 \cdot A_{7}$ & $L$ & $2 A$ & 4 & 1 \\
\hline 6 & $6 \cdot P S L_{3}(4)$ & $L$ & $2 A$ & 4 & 1 \\
\hline 6 & $S U_{3}(3)$ & $L \times 2, L \times 3$ & $2 A$, resp. $3 A$ & 4 , resp. 3 & 1 \\
\hline 6 & $S U_{4}(2)$ & $L, L \times 2$, or $L \times 3$ & $2 A B$, resp. $3 A B, 3 C$ & 4, resp. 3,3 & 1 \\
\hline 6 & $6_{1} \cdot P S U_{4}(3)$ & $L$ & $2 A$, resp. $3 A, 3 B$ & 4, resp. 3,3 & 1 \\
\hline 6 & $2 \cdot J_{2}$ & $L, L \times 3$ & $2 A$, resp. $3 A$ & 4 , resp. 3 & 1 \\
\hline 7 & $S p_{6}(2)$ & $L \times 2$ & $2 A$ & 2 & $1 / 2$ \\
\hline 7 & $S U_{3}(3)$ & $L$ & $2 A$ & 4 & 1 \\
\hline 7 & $S p_{6}(2)$ & $L$ & $2 C$, resp. $3 A$ & 4 , resp. 3 & 1 \\
\hline 8 & $2 \cdot \Omega_{8}^{+}(2)$ & $L \cdot 2$ & $2 F$ & 2 & $1 / 2$ \\
\hline 8 & $2 \cdot \Omega_{8}^{+}(2)$ & $L$ & $2 B$, resp. $3 A$ & 4 , resp. 3 & 1 \\
\hline 10 & $S U_{5}(2)$ & $L \times 2$ & $2 A$ & 4 & 1 \\
\hline$n-1$ & $\mathrm{~A}_{n}$ & $\mathrm{~S}_{n}$ & 2-cycle & 2 & $1 / 2$ \\
\hline$n-1$ & $\mathrm{~A}_{n}$ & $L$ & $(1,2,3)$, resp. $(1,2)(3,4)$ & 3 , resp. 4 & 1 \\
\hline
\end{tabular}




\section{Linear groups generated by elements of bounded deviation}

In this section we will prove Theorem 1.1. The standing hypothesis throughout the section is that

$(\star \star) V=\mathbb{C}^{d}, d>1, G$ is a finite irreducible subgroup of $\mathcal{G}=G L(V), \mathcal{X} \subset \mathcal{G}, C \geq 4$ a given constant, $Z(\mathcal{G}) G=\langle\mathcal{X}\rangle$, and $d_{2}(g)^{2} \leq C$ for all $g \in \mathcal{X}$.

Let $\chi$ denote the character of $Z(\mathcal{G}) G$ afforded by $V$.

\subsection{The imprimitive case}

Here we consider the case where $G$ (transitively) permutes the $m$ summands of a decomposition $V=V_{1} \oplus \cdots \oplus V_{m}, m>1$. For any $g \in \mathcal{X}$, let $\mu(g)$ denote the number of subspaces $V_{i}$ that are moved by $g$. Then $g$ fixes (setwise) precisely $m-\mu(g)$ subspaces $V_{i}$, whence $|\chi(g)| \leq(m-\mu(g)) \operatorname{dim}\left(V_{1}\right)$. It follows that

$$
C \geq d_{2}(g)^{2}=2(\chi(1)-|\chi(g)|) \geq 2 \mu(g) \operatorname{dim}\left(V_{1}\right) .
$$

Since $Z(\mathcal{G}) G=\langle\mathcal{X}\rangle$, there must be some $g \in \mathcal{X}$ which acts non-trivially on the set $\left\{V_{1}, \ldots, V_{m}\right\}$, for which $\mu(g) \geq 2$. Thus $\operatorname{dim}\left(V_{1}\right) \leq C / 4$ (also see Example 4.1 for a partial converse).

Now we choose a $G$-invariant decomposition $V=V_{1} \oplus \cdots \oplus V_{m}$ with $m>1$ smallest possible. This means that the induced action $\pi$ of $G$ on the set $\left\{V_{1}, \ldots, V_{m}\right\}$ is primitive. Assume in addition that $(G, V)$ does not satisfy the conclusion (iii) of Theorem 1.1, i.e. $\pi(G) \geq \mathrm{A}_{m}$. By [LS, Corollary 3], $\mu(g)>2(\sqrt{m}-1)$ (for some $g \in \mathcal{X}$ ). On the other hand, as shown above, $1 \leq k:=\operatorname{dim}\left(V_{1}\right) \leq C / 4$. Now (7) implies that $m<(1+C / 4 k)^{2}$ and so $\operatorname{dim}(V)<k(\overline{1}+C / 4 k)^{2}=: h(\bar{k})$. Since the function $h(k)$ is decreasing on [1,C/4], we see that $\operatorname{dim}(V)<h(1)=(1+C / 4)^{2}$. Thus we have shown that if $G$ is as in $(\star \star)$ and $G$ is imprimitive, then either $G$ satisfies Theorem 1.1(iii), or $\operatorname{dim}(V)<(1+C / 4)^{2}$. Observe that $(1+C / 4)^{2}<\mathfrak{f}(C)$ as $C \geq 4$. Hence we have proved Theorem 1.1 in the case $G$ is imprimitive.

Example 4.1. Let $C \geq 4, V_{1}=\mathbb{C}^{k}$ with $1 \leq k \leq C / 4$, and let $H \leq G L\left(V_{1}\right)$ be any subgroup generated by $\left\{\left[x^{-1} h x, y\right] \mid x, y \in \bar{H}\right\}$ for a fixed element $h \in H$ (for instance, one can consider any quasi-simple subgroup $H$ and any $h \in H \backslash Z(H)$ ). Then for any $m \geq 2$, the subgroup $G=H \geq \mathrm{S}_{m}<G L_{m k}(\mathbb{C})$ is generated by $g^{G}$ for some element $g \in G$ satisfying $d_{2}(g)^{2} \leq C$. Indeed, we may write $V=\mathbb{C}^{m k}$ as the set of $m$-tuples $\left(v_{1}, \ldots, v_{m}\right), v_{i} \in V_{1}$, and define $g$ via $g\left(v_{1}, v_{2}, v_{3}, \ldots, v_{m}\right)=\left(v_{2}, h\left(v_{1}\right), v_{3}, \ldots, v_{m}\right)$; in particular, $\operatorname{Tr}(g)=(m-2) k$ and so $d_{2}(g)^{2}=2(\operatorname{dim}(V)-|\operatorname{Tr}(g)|)=4 k \leq C$. For any $x, y \in H$, observe that $G$ contains the elements

$$
\begin{aligned}
\tilde{x}:\left(v_{1}, v_{2}, \ldots, v_{m}\right) & \mapsto\left(x\left(v_{1}\right), v_{2}, \ldots, v_{m}\right), \\
\tilde{y}:\left(v_{1}, v_{2}, \ldots, v_{m}\right) & \mapsto\left(y\left(v_{1}\right), v_{2}, \ldots, v_{m}\right) .
\end{aligned}
$$

Then $K:=\left\langle g^{G}\right\rangle$ contains the element $\left[\tilde{x}^{-1} g^{2} \tilde{x}, \tilde{y}\right]=\left(\tilde{x}^{-1} g \tilde{x}\right)^{2} \cdot\left(\tilde{y} \tilde{x}^{-1} \cdot g \cdot \tilde{x} \tilde{y}^{-1}\right)^{-2}$ which acts on $V$ via $\left(v_{1}, v_{2}, \ldots, v_{m}\right) \mapsto\left(\left[x^{-1} h x, y\right]\left(v_{1}\right), v_{2}, \ldots, v_{m}\right)$. The assumption 
on $H$ now implies that $K>H \times 1 \times \cdots \times 1$. But $g$ induces the transposition $(1,2)$ while acting on the $m$-tuples $\left(v_{1}, \ldots, v_{m}\right), v_{i} \in V_{1}$. Hence $K>H^{m}$ and $K / H^{m} \cong \mathrm{S}_{m}$, and so $K=G$ as stated.

\subsection{Tensor decomposable case}

Here we assume that $G$ is primitive but tensor decomposable on $V: \chi=\alpha_{1} \otimes \cdots \otimes \alpha_{n}$, where $n \geq 2, \alpha_{i} \in \operatorname{Irr}(G)$ is primitive and tensor indecomposable for each $i$, and $\alpha_{1}(1) \geq$ $\cdots \geq \alpha_{n}(1) \geq 2$. Then we can find $g \in \mathcal{X}$ such that $d_{2}(g)^{2} \leq C$ and $\left|\alpha_{n}(g)\right|<\alpha_{n}(1)$. In the case $\beta:=\alpha_{n}$ is tensor induced, among such elements $g$ we can find one that acts non-trivially on the set of tensor factors of $\beta$ (as otherwise $\left.\beta\right|_{G}$ would be tensor decomposable). By Theorem 1.3 applied to $(G, \beta, g), \beta(1)-|\beta(g)| \geq \delta:=(3-\sqrt{5}) / 2$; moreover, if $\beta(1) \geq 13$ then $\beta(1)-|\beta(g)| \geq 2$. In the latter case,

$$
C \geq 2(\alpha(1) \beta(1)-|\alpha(g) \beta(g)|) \geq 2 \alpha(1)(\beta(1)-|\beta(g)|) \geq 4 \alpha(1),
$$

and so $\alpha(1) \leq C / 4$, where $\alpha:=\alpha_{1} \otimes \cdots \otimes \alpha_{n-1}$. By the choice of $\alpha_{n}$, we get $\beta(1) \leq \alpha(1)$ and so $\chi(1) \leq C^{2} / 16<\mathfrak{f}(C)$. It remains to consider the case $2 \leq \beta(1) \leq 12$. Then

$$
C \geq 2(\alpha(1) \beta(1)-|\alpha(g) \beta(g)|) \geq 2 \alpha(1)(\beta(1)-|\beta(g)|) \geq 2 \delta \alpha(1),
$$

and so $\alpha(1) \leq C / 2 \delta$. Therefore,

$$
\chi(1) \leq 12 \cdot C / 2 \delta=12 C /(3-\sqrt{5})<16 C<\mathfrak{f}(C),
$$

and we are done.

Example 4.2. Given any $C \geq 4$, choose $m=1+\lfloor C / 4\rfloor$. Let $S_{m}$ act on $\mathbb{C}^{m-1}$ as on its deleted natural permutation module. This induces a natural action of $G=\mathrm{S}_{m} \times \mathrm{S}_{m}$ on $V=\mathbb{C}^{m-1} \otimes \mathbb{C}^{m-1}$. Consider the element $g_{1}=(\tau, 1)$ and $g_{2}=(1, \tau)$, where $\tau$ is the transposition $(1,2) \in S_{m}$. Then $\chi(1)=(m-1)^{2}, \chi\left(g_{i}\right)=(m-1)(m-3)$ and so $d_{2}\left(g_{i}\right)^{2}=4(m-1)$. By the choice of $m$, we see that $d_{2}\left(g_{i}\right)^{2} \leq C$ is very close to $C$ and $\operatorname{dim}(V)$ is very close to $C^{2} / 16$. Clearly, $G$ satisfies the set-up ( $\left.\star \star\right)$ with $\mathcal{X}:=g_{1}^{G} \cup g_{2}^{G}$. (Adding to $G$ an involution inverting the two factors $\mathbb{C}^{m-1}$ of $V$, we then have $G=\left\langle g_{1}^{G}\right\rangle$.)

\subsection{Tensor induced case}

Consider the case where $G$ is tensor induced on $V: V=V_{1} \otimes \cdots \otimes V_{m}$, with $\operatorname{dim}\left(V_{i}\right)=$ $a>1$ and $G$ permutes the $m$ tensor factors $V_{1}, \ldots, V_{m}$ (transitively). Then we can find $g \in \mathcal{X}$ such that $d_{2}(g)^{2} \leq C$ and $g$ acts non-trivially on $\left\{V_{1}, \ldots, V_{m}\right\}$. By Lemma 2.25, $|\chi(g)| \leq a^{m-1} \leq \chi(1) / 2$. Hence $C \geq 2(\chi(1)-|\chi(g)|) \geq \chi(1)$.

\subsection{Extraspecial case}

Here we consider the case (iii) of [GT3, Proposition 2.8]. In this case, $\operatorname{dim}(V)=p^{m}$ for some prime $p$ and some integer $m \geq 2$. Pick any $g \in \mathcal{X} \backslash Z(\mathcal{G})$. By [GT1, Lemma 2.4], $|\chi(g)| \leq p^{m-1 / 2} \leq \chi(1) / \sqrt{2}$. Thus $C \geq 2(\chi(1)-|\chi(g)|) \geq \chi(1)(2-\sqrt{2})$ and so $\chi(1) \leq C(1+1 / \sqrt{2})$. 


\subsection{Almost quasi-simple groups}

Here we consider the case where $G$ is a finite almost quasi-simple group that satisfies $(\star \star)$. In particular, $L:=G^{(\infty)}$ is quasi-simple and $L / Z(L)$ is the unique non-abelian composition factor of $G$. By the above, we may assume in addition that $G$ is primitive and tensor indecomposable on $V$. Since $d>1$ and $Z(\mathcal{G}) G=\langle\mathcal{X}\rangle$, there exists $g \in G \backslash Z(G)$ with $d_{2}(g)^{2} \leq C$. By Lemma 2.19 and its proof, $L$ acts irreducibly on $V$ and there is $h \in L \backslash Z(L)$ such that $d_{2}(h)^{2} \leq 4 C$.

First assume that $L ¥ \mathrm{~A}_{n}$ for any $n \geq 10$. Then by Proposition 2.22(i) and Lemma 2.23, $|\chi(h) / \chi(1)| \leq 19 / 20$. It follows that $4 C \geq d_{2}(h)^{2}=2(\chi(1)-|\chi(h)|) \geq \chi(1) / 10$, and $\operatorname{so} \operatorname{dim}(V) \leq 40 C \leq \mathfrak{f}(C)$.

We may now assume that $L=\mathrm{A}_{n}$ for some $n \geq 10$, and moreover $\left.V\right|_{L}$ is not isomorphic to the deleted permutation module of $L$ (as otherwise $(G, V)$ satisfies Theorem 1.1(ii)). Up to scalars we may also assume that $\mathrm{A}_{n} \leq G \leq \mathrm{S}_{n}$. By Proposition 2.22(ii), $|\chi(g) / \chi(1)| \leq 1 / 2+(n-1) / 2 n=1-1 / 2 n$, whence

$$
C \geq d_{2}(g)^{2}=2(\chi(1)-|\chi(g)|) \geq \chi(1) / n .
$$

In particular, if $n \leq 40$, then $\chi(1) \leq 40 C \leq \mathfrak{f}(C)$. Henceforth we may assume that $n \geq 41$.

Now we choose $\lambda \vdash n$ such that $\left.\chi\right|_{L}$ is an irreducible constituent of $\left.\rho^{\lambda}\right|_{L}$, where $\rho^{\lambda} \in \operatorname{Irr}\left(\mathrm{S}_{n}\right)$ is labeled by $\lambda$, and apply Lemma 3.2 to $\rho^{\lambda}$. Assume we are in the former case of Lemma 3.2(ii). Then $\chi(1) \geq \rho^{\lambda}(1) / 2 \geq n(n-1)(n-2)(n-7) / 48>26 n^{2}$ as $n \geq 41$. Together with (8), we now have $26 n^{2}<\chi(1) \leq n C$. It follows that $n \leq C / 26$ and so $\chi(1)<C^{2} / 26<\mathfrak{f}(C)$.

It therefore remains to consider the case $\lambda \in R_{n}(2) \cup R_{n}(3)$; in particular, $\chi_{L}=\left.\rho^{\lambda}\right|_{L}$.

Consider the case $\lambda \in R_{n}(2)$. By Lemma 3.3 and its proof,

$$
C \geq 2(\chi(1)-|\chi(g)|) \geq 2(\chi(1)-|\chi(\mathbf{t})|) \geq 4 n-12 \geq 152,
$$

whence $n \leq 3+C / 4$. Thus $\chi(1) \leq(n-1)(n-2) / 2 \leq(1+C / 4)(2+C / 4) / 2 \leq \mathfrak{f}(C)$.

Finally, assume that $\lambda \in R_{n}(3)$. By Lemma 3.3 and its proof,

$$
C \geq 2(\chi(1)-|\chi(g)|) \geq 2(\chi(1)-|\chi(\mathbf{t})|) \geq 2(n-2)(n-5)>68 n,
$$

whence $n<C / 68$. Hence (8) implies that $\chi(1) \leq n C<C^{2} / 68<\mathfrak{f}(C)$. Thus we have proved Theorem 1.1 in case $G$ is almost quasi-simple (and primitive, tensor indecomposable on $V$ ). By [GT3, Proposition 2.8], we have therefore completed the proof of Theorem 1.1.

\section{Linear groups generated by elements of age $\leq 1$}

In this section we will address the following situation:

(๑) $V=\mathbb{C}^{d}, d>1, G$ is a finite irreducible subgroup of $\mathcal{G}=G L(V), \mathcal{X} \subset \mathcal{G}$, $Z(\mathcal{G}) G=\langle\mathcal{X}\rangle$, and $0<\operatorname{age}^{*}(g) \leq 1$ for all $g \in \mathcal{X}$. 
By Corollary 2.18, such groups $G$ satisfy the set-up ( $\star \star)$ of $\S 4$ with $C:=9.111$. We will denote by $\chi$ the character of $Z(\mathcal{G}) G$ afforded by $V$, and frequently refer to intermediate results established in $\$ 4$.

\subsection{Imprimitive groups}

First we record the following easy observation:

Lemma 5.1. Let a finite subgroup $G<G L(V)$ preserve a decomposition $W=V_{1} \oplus$ $\cdots \oplus V_{n}$ of a subspace $W \subseteq V$, with $\operatorname{dim}\left(V_{i}\right)=1$ and $G$ inducing either $\mathrm{S}_{n}$ or $\mathrm{A}_{n}$ while permuting the $n$ subspaces $V_{1}, \ldots, V_{n}$. Then for any element $g \in G$ with age* $(g) \leq 1$, one of the following holds.

(i) g acts either trivially, or as a 2-cycle on $\left\{V_{1}, \ldots, V_{n}\right\}$.

(ii) Some scalar multiple $\mu \mathrm{g}$ of $g$ is a complex bireflection of order 2 or 3 on $V$, and $g$ acts as a 3-cycle, or a double transposition on $\left\{V_{1}, \ldots, V_{n}\right\}$. Furthermore, age* $(g)=1$.

Proof. Observe that, if $h$ acts as an $m$-cycle on $\left\{V_{1}, \ldots, V_{m}\right\}$, then it has minimal polynomial $t^{m}-\alpha$, and $m$ eigenvalues $\beta e^{2 \pi i j / m}, 0 \leq j \leq m-1$, on $V_{1} \oplus \cdots \oplus V_{m}$, for some $\alpha, \beta \in \mathbb{C}^{\times}$. In particular,

$$
\operatorname{age}^{*}\left(\left.h\right|_{V_{1} \oplus \cdots \oplus V_{m}}\right) \geq(m-1) / 2 .
$$

Now assume $g \in G$ has age* $(g) \leq 1$ but $g$ does not satisfy (i). By Lemma 2.2(ii), there is $\mu \in S^{1}$ such that age $(\mu g) \leq 1$. By Corollary $2.18, d_{2}(g)^{2} \leq(2.9) \pi$, and so $g$ cannot move more than four subspaces $V_{i}$ by (7). Thus $g$ acts as a 3-cycle, a double transposition, or a 4-cycle on $\left\{V_{1}, \ldots, V_{n}\right\}$. In the third case, age* $(g)>1$ by (9). In the first two cases, (9), Lemma 2.2(iii), and the condition age $(\mu g) \leq 1$ force $\mu g$ to act as a complex bireflection of order 3, resp. 2 , on $W$, and trivially on a complement $U$ to $W$ in $V$. The last claim in (ii) now follows from (9) and the assumption that age* $(g) \leq 1$.

Lemma 5.2. Let $G<G L(V)$ be as in (\$). Assume that $G$ preserves a decomposition $V=V_{1} \oplus \cdots \oplus V_{n}$, with $n>1$ smallest possible. Let $\pi$ denote the induced permutation action of $Z(\mathcal{G}) G$ on $\left\{V_{1}, \ldots, V_{n}\right\}$. Then one of the following holds:

(i) $\operatorname{dim}\left(V_{i}\right)=1$, and $(\pi(G), n)=\left(\mathrm{S}_{n}, n\right),\left(\mathrm{A}_{n}, n\right),\left(A S L_{3}(2), 8\right),\left(S L_{3}(2), 7\right),\left(\mathrm{A}_{5}, 6\right)$, $\left(D_{10}, 5\right)$. If $g \in \mathcal{X}$ and $\pi(g)$ is not 1 nor a 2-cycle, then $g$ is a complex bireflection of order 2 or 3 and age* $(g)=1$.

(ii) $\operatorname{dim}\left(V_{i}\right)=2$, age* $(g)=1$ for any $g \in \mathcal{X}$ with $\pi(g) \neq 1$, and the conclusion (iii) of Theorem 1.4 holds.

Proof. Our assumptions imply that $\pi(G)$ is a primitive subgroup of $\mathrm{S}_{n}$, and that there must be some $h \in \mathcal{X}$ that moves $\mu(g) \geq 2$ subspaces $V_{i}$. By $(7), 2 \mu(h) \operatorname{dim}\left(V_{1}\right) \leq 9.111$ and so $\mu(h) \operatorname{dim}\left(V_{1}\right) \leq 4$; in particular, $\operatorname{dim}\left(V_{1}\right)=1$ or 2 . Consider the former case: $\operatorname{dim}\left(V_{1}\right)=1$; in particular, $\mu(t) \leq 4$ for all $t \in \mathcal{X}$. If $\mu(t)=2$, resp. 3, for some $t \in \mathcal{X}$, then $\pi(G)$ is a primitive permutation group containing a 2-cycle, resp. a 3-cycle, whence $\pi(G)=\mathrm{S}_{n}$ or $\mathrm{A}_{n}$ by [W, Theorem 13.3]. Otherwise $\pi(t)$ is a double transposition for 
all $t \in \mathcal{X}$ with $\pi(t) \neq 1$. Thus $\pi(G)$ is a primitive subgroup of $\mathrm{S}_{n}$ generated by some double transpositions. Assume in addition that $\pi(G) \nsucceq \mathrm{A}_{n}$. Then $4>2(\sqrt{n}-1)$ by [LS, Corollary 3], i.e. $n \leq 8$, and we arrive at the primitive permutation groups listed in (i). The second claim in (i) follows from Lemma 5.1(ii).

In the latter case, $\mu(h)=2$, i.e. $h$ is a transposition. This conclusion in fact holds for any $g \in \mathcal{X}$ with $\pi(g) \neq 1$. Thus $\pi(G)$ is a primitive permutation group generated by transpositions, and so $\pi(G)=\mathrm{S}_{n}$. Let $D:=\operatorname{Ker}(\pi)$ and consider any $g \in G \backslash D$ with age $(g) \leq 1$. Then we may assume that $g: V_{1} \leftrightarrow V_{2}$ and $g\left(V_{j}\right)=V_{j}$ for all $j \geq 3$. It is not difficult to see that $\operatorname{Spec}\left(\left.g\right|_{V_{1} \oplus V_{2}}\right)$ is the union of two cosets of $C_{2}=\langle-1\rangle$ in $S^{1}$. By Lemma 2.20 (with $m=2$ ) and Lemma 2.2(iii), the condition age $(g) \leq 1$ implies that $g^{2}=1_{V}$, age $(g)=1, g$ is trivial on each $V_{j}$ with $j \geq 3$; in particular, $g$ is a bireflection. This argument also shows that age* $(t)=1$ for all $t \in \mathcal{X}$ with $\pi(t) \neq 1$. We will apply this observation to suitable inverse images (in $G$ ) of transpositions $(i, i+1), 1 \leq i \leq n-1$, of $S_{n}$ to show that $G$ is a split extension of $D$ by $S_{n}$. Indeed, denote the element $g$ we have just analyzed by $g_{1}$. For any $1 \leq i \leq n-1$, a $G$-conjugate $g_{i}$ of $g_{1}$ will project onto the transposition $(i, i+1)$ and have age $=1$. Hence our observation (applied to $g_{i}$ ) yields

$$
g_{i}: V_{i} \leftrightarrow V_{i+1}, \quad g_{i}^{2}=1_{V},\left.\quad g_{i}\right|_{V_{j}}=1_{V_{j}} \quad \text { for } j \neq i, i+1 .
$$

Clearly, $\left(g_{i} g_{j}\right)^{2}=1_{V}$ if $|i-j|>1$. Next, if $v \in V_{i}$ then

$$
\left(g_{i} g_{i+1}\right)^{3}: v \stackrel{g_{i+1}}{\longmapsto} v \stackrel{g_{i}}{\longmapsto} g_{i}(v) \stackrel{g_{i+1}}{\longmapsto} g_{i+1} g_{i}(v) \stackrel{g_{i}}{\longmapsto} g_{i+1} g_{i}(v) \stackrel{g_{i+1}}{\longmapsto} g_{i}(v) \stackrel{g_{i}}{\longmapsto} v,
$$

and similarly for all $v \in V_{j}$ with $j \neq i$, whence $\left(g_{i} g_{i+1}\right)^{3}=1_{V}$. Thus $H=\left\langle g_{1}, \ldots, g_{n-1}\right\rangle$ is a quotient of $\mathrm{S}_{n}$, and $D H=G=D \cdot \mathrm{S}_{n}$. It follows that $H \cong \mathrm{S}_{n}$. In fact, one can find a basis $\left(u_{i}, v_{i}\right)$ of each $V_{i}$ such that $H$ acts via permuting the indices of the $u_{i}$ 's, resp. of the $v_{i}$ 's:

$$
g_{i}: u_{i} \leftrightarrow u_{i+1}, \quad v_{i} \leftrightarrow v_{i+1}, \quad u_{j} \mapsto u_{j}, \quad v_{j} \mapsto v_{j}, \quad \text { for } j \neq i, i+1 .
$$

Lemma 5.3. Let $G<G L(V)$ be a finite irreducible subgroup. Assume that $G$ preserves a decomposition $V=V_{1} \oplus \cdots \oplus V_{n}$, with $\operatorname{dim}\left(V_{i}\right)=1$ and $G$ inducing either $\mathrm{S}_{n}$ or $\mathrm{A}_{n}$ while permuting the $n$ subspaces $V_{1}, \ldots, V_{n}$, and that $n \geq 10$. Then $G$ contains a complex bireflection of order 3.

Proof. We represent elements of $G$ by their matrices with respect to a basis $\left(e_{1}, \ldots, e_{n}\right)$ with $V_{i}=\left\langle e_{i}\right\rangle_{\mathbb{C}}$. Let $D$ be the normal subgroup of $G$ consisting of diagonal elements, so that $\mathrm{S}_{n} \geq G / D \triangleright A:=\mathrm{A}_{n}$. Notice that, as an $A$-module, every chief factor of $D$ is either the trivial module $\mathcal{I}$, or the heart $\mathcal{H}$ of the natural permutation module, in characteristic $p$ for some prime $p$. It is well known that $H^{2}(A, \mathcal{I})=0$ if $n \geq 8$ and $p>2$. Furthermore, $H^{2}(A, \mathcal{H})=0$ if $n \geq 10$ by the main result of [KP]. It follows that $O_{2^{\prime}}(D) \cdot A$ splits over $O_{2^{\prime}}(D)$. Since $D=O_{2}(D) \times O_{2^{\prime}}(D)$, we may assume that $G$ contains a subgroup $H=O_{2}(D) \cdot A$. 
Now inside $H$ we can find an inverse image $g$ of order 3 of a 3 -cycle in $A$. We may assume that $g=\operatorname{diag}\left(\left(\begin{array}{c}{ }_{c}{ } b \\ c\end{array}\right), d_{1}, \ldots, d_{n-3}\right)$, where $a b c=1$ and $d_{i}^{3}=1$. Since $n-3 \geq 4$, we may also assume that $d_{1}=d_{2}$. Next, in $H$ we can find an element $h=\operatorname{diag}\left(\left(y^{x}\right), z,\left(v^{u}\right), w_{1}, \ldots, w_{n-5}\right)$. Then $[g, h]=\operatorname{diag}\left(\left(\begin{array}{cc}b^{\prime} & a^{\prime} \\ & c^{\prime}\end{array}\right), 1, \ldots, 1\right)$, where $a^{\prime} b^{\prime} c^{\prime} \neq 0$; in particular, it permutes $V_{1}, V_{2}, V_{3}$ cyclically. Notice that, $[g, h] \equiv$ $g^{-1}\left(\bmod O_{2}(D)\right)$ and so $[g, h] \in O_{2}(D) \cdot C_{3}$, where $C_{3}=\langle g\rangle$. Hence, a suitable 2power $t$ of $[g, h]$ has order 3 , acts as a 3 -cycle on $\left\{V_{1}, V_{2}, V_{3}\right\}$, and fixes every $e_{i}$ with $i \geq 4$. Clearly, such a $t$ is conjugate to $\operatorname{diag}\left(e^{2 \pi i / 3}, e^{4 \pi i / 3}, 1, \ldots, 1\right)$ and so it is a complex bireflection of order 3 .

Lemma 5.4. Let $\mathcal{T}=G L_{1}(\mathbb{C})^{n}$ be a maximal torus of $\mathcal{G}=G L_{n}(\mathbb{C})$, so that $N:=$ $N_{\mathcal{G}}(\mathcal{T})=\mathcal{T} \cdot \mathrm{S}_{n}$, and $n \geq 5$. Assume $D<\mathcal{T}$ is a finite subgroup which is normalized by the subgroup $\mathcal{T} \cdot \mathrm{A}_{n}$ of $N$. Then $D \triangleleft N$.

Proof. 1) Without loss we may assume that $D$ is a $p$-group for some prime $p$. If $\exp (D)$ $=q=p^{c}$, then $D$ is contained in $T:=\left\{x \in \mathcal{T} \mid x^{p^{c}}=1\right\}$. Using the additive notation, we may identify $T$ with the natural permutation $R \mathrm{~S}_{n}$-module $M:=\left\langle e_{1}, \ldots, e_{n}\right\rangle_{R}$, where $R:=\mathbb{Z} / q \mathbb{Z}$. It suffices now to prove that any $\mathrm{A}_{n}$-submodule $N$ of $M$ is $\mathrm{S}_{n}$-invariant.

2) Assume that $p \nmid n$, with $n \geq 5$ or $(n, p)=(4,5)$, and consider the module $L:=$ $\left\{\sum_{i=1}^{n} a_{i} e_{i} \mid a_{i} \in R, \sum_{i=1}^{n} a_{i}=0\right\}$. Let $\mathcal{H}$ denote the heart of the natural permutation $\mathbb{F}_{p} \mathrm{~S}_{n}$-module. The condition on $(n, p)$ implies that $\mathcal{H}$ is irreducible over $\mathrm{A}_{n}$. Now observe that the $A_{n}$-module $L$ is uniserial, with $\mathcal{H}$ as the unique composition factor. Hence any $\mathrm{A}_{n}$-submodule $Y$ of $L$ is $\mathrm{S}_{n}$-invariant. (Indeed, if $t \in \mathrm{S}_{n}$ then the $\mathrm{A}_{n}$-modules $Y$ and $t Y$ have same composition length and so $Y=t Y$ as $L$ is uniserial.)

3) Consider the case $p \nmid n$. Then $M=A \oplus B$ as $S_{n}$-modules, where $A=\left\langle\sum_{i=1}^{n} e_{i}\right\rangle_{R}$, and $B=\left\{\sum_{i=1}^{n} a_{i} e_{i} \mid a_{i} \in R, \sum_{i=1}^{n} a_{i}=0\right\}$. By the result of 2) applied to the $\mathrm{S}_{n^{-}}$ module $L:=B$, any $\mathrm{A}_{n}$-submodule $Y$ of $B$ is $\mathrm{S}_{n}$-invariant. On the other hand, $\mathrm{S}_{n}$ acts trivially on $A$, whence any $\mathrm{A}_{n}$-submodule $X$ of $A$ is obviously $\mathrm{S}_{n}$-invariant. Now set $X:=N \cap A$ and $Y:=N \cap B$. Observe that any composition factor of the $\mathrm{A}_{n}$-module $N /(X \oplus Y)$ is a common composition factor of $M / A \cong B$ and $M / B \cong A$. Hence $N=X \oplus Y$ and so it is $\mathrm{S}_{n}$-invariant.

4) Finally, we assume $p \mid n$ and consider the natural subgroups $A_{n-1}$ and $S_{n-1}$ in $S_{n}$, which fix $e_{1}$. Then $M=A \oplus B$ as $\mathrm{S}_{n-1}$-modules, where $A=\left\langle e_{1}, \sum_{i=2}^{n} e_{i}\right\rangle_{R}$, and $B=\left\{\sum_{i=2}^{n} a_{i} e_{i} \mid a_{i} \in R, \sum_{i=2}^{n} a_{i}=0\right\}$. Then the conclusion of 2) applied to the $\mathrm{S}_{n-1}$ module $L:=B$ implies that any $\mathrm{A}_{n-1}$-submodule $Y$ of $B$ is $\mathrm{S}_{n-1}$-invariant. Also, $\mathrm{S}_{n-1}$ acts trivially on $A$, whence any $\mathrm{A}_{n-1}$-submodule $X$ of $A$ is obviously $\mathrm{S}_{n-1}$-invariant. Now set $X:=N \cap A$ and $Y:=N \cap B$. As in 3), we see that $N=X \oplus Y$, and so it is $\mathrm{S}_{n-1}$-invariant. Thus $N$ is invariant under $\left\langle\mathrm{A}_{n}, \mathrm{~S}_{n-1}\right\rangle=\mathrm{S}_{n}$.

One of the main results of this subsection is the following

Theorem 5.5. Let $G<\mathcal{G}:=G L(V)$ be a finite irreducible subgroup that preserves a decomposition $V=V_{1} \oplus \cdots \oplus V_{n}$, with $n>1$ smallest possible. Assume in addition that $n \geq 3$ and $\operatorname{dim}\left(V_{i}\right)=1$. 
(i) Assume $G$ satisfies $(\boldsymbol{M})$ and contains a non-central element $g$ with $0<\operatorname{age*}(g)<1$. Assume in addition that $\pi(G) \geq \mathrm{A}_{n}$, where $\pi$ denotes the permutation action of $G$ on $\left\{V_{1}, \ldots, V_{n}\right\}$. Then there is a finite subgroup $Z<Z(\mathcal{G})$ such that $Z G$ contains a complex reflection.

(ii) If $(G, V)$ is a basic non-RT pair, then there is a finite subgroup $Z<Z(\mathcal{G})$ and a complex reflection group $H=G(d, 1, n)$ with $d>1$ (in the notation of [ST]) such that $Z G=Z H$. Conversely, any $G(d, 1, n)$ with $d>1$ yields a basic non-RT pair.

Proof. Fix a basis vector $e_{i}$ for each $V_{i}$, and let $D:=\operatorname{Ker}(\pi) \triangleleft G$ consist of all the elements of $G$ that act diagonally on the basis $\left(e_{1}, \ldots, e_{n}\right)$.

1) Here we show that if there is an element $g \in G \backslash D$ with age $(g)<1$, then either

(a) D contains a non-scalar element $h$ with age $(h)<1$, or

(b) $\lambda g$ is a reflection for some $\lambda=e^{-2 \pi i t}$, with $0 \leq t<1 / 2 n$ and $\lambda^{2} \cdot 1_{V} \in G$.

For, by Lemma 5.1, $g$ has the matrix $\operatorname{diag}\left(\left(\begin{array}{ll}0 & a \\ b & 0\end{array}\right), c_{3}, \ldots, c_{n}\right)$ in the given basis, for some $a, b, c_{i} \in \mathbb{C}^{\times}$. Then $\operatorname{Spec}(g)=\left\{\sqrt{a b},-\sqrt{a b}, c_{3}, \ldots, c_{n}\right\}$. Since $g$ has finite order, we may write $c_{j}=e^{2 \pi i r_{j}}$ with $0 \leq r_{j}<1$ for $j>2$ and $\{\sqrt{a b},-\sqrt{a b}\}=$ $\left\{e^{2 \pi i r_{1}}, e^{2 \pi i\left(r_{1}+1 / 2\right)}\right\}$ with $0 \leq r_{1}<1 / 2$. By our assumptions, $1>\operatorname{age}(g)=1 / 2+$ $2 r_{1}+\sum_{i=3}^{n} r_{i}$, and so $2 r_{1}+\sum_{i=3}^{n} r_{i}<1 / 2$. Observe that $g^{2}=\operatorname{diag}\left(a b, a b, c_{3}^{2}, \ldots, c_{n}^{2}\right)$ and age $\left(g^{2}\right) \leq 4 r_{1}+2 \sum_{i=3}^{n} r_{i}<1$. Now if $g^{2}$ is non-scalar, then we can set $h=g^{2}$. Assume $g^{2}$ is scalar; in particular, $a b=c_{i}^{2}$ for all $i>2$ and $c_{3}^{2} \cdot 1_{V}=g^{2} \in G$. Notice that $c_{3}$ has finite order in $S^{1}$ as $|g|$ is finite. Then $\operatorname{Spec}\left(c_{3}^{-1} g\right)=\{1,-1,1, \pm 1, \ldots, \pm 1\}$. By Lemma 2.21 (with $m=2$ ), the condition age $(g)<1$ now implies that $\operatorname{Spec}\left(c_{3}^{-1} g\right)=$ $\{1,-1,1, \ldots, 1\}$, and so $c_{3}^{-1} g$ is a reflection. Finally, $\operatorname{Spec}(g)=\left\{-c_{3}, c_{3}, \ldots, c_{3}\right\}$ and age $(g)<1$, so $c_{3}=e^{2 \pi i t}$ with $0 \leq t<1 / 2 n$. Thus $\lambda:=c_{3}^{-1}$ has the properties specified in (b).

2) Now we consider the situation of (ii). Then $G$ contains some non-central element $g$ with age $(g)<1$ such that $G=\left\langle g^{G}\right\rangle$. Since $G$ is irreducible and $n \geq 3, G \neq D$, and so $g \notin D$. Now we can apply the result of 1) to the element $g$. In the case $D \ni h$ with $h$ non-scalar and age $(h)<1$, we would have $G=\left\langle h^{G}\right\rangle \leq D$ (as $(G, V)$ is a basic nonRT pair), a contradiction. Hence $\lambda g$ is a reflection for some $\lambda$ as specified in (b). Since $G=\left\langle g^{G}\right\rangle$, we see that $Z G=Z H$ for $Z=\left\langle\lambda \cdot 1_{V}\right\rangle\left\langle Z(\mathcal{G})\right.$ and $H=\left\langle(\lambda g)^{G}\right\rangle$ is a finite group generated by reflections. Since the c.r.g. $H$ acts imprimitively on $V$ (inducing $S_{n}$ on $\left.\left\{V_{1}, \ldots, V_{n}\right\}\right)$, by [ST] we must have $H=G(d e, e, n)$ for some positive integers $d, e$. Assume $e>1$. Then $Z G$ contains non-central elements $r:=\operatorname{diag}\left(1, \ldots, 1, e^{2 \pi i / e}\right)$ and $\lambda^{-1} r$, with age $(r)=1 / e \leq 1 / 2$ and $\operatorname{age}\left(\lambda^{-1} r\right)=1 / e+n / t<1$. If $\lambda \cdot 1_{V} \in G$, then $G=Z G$ contains $r$. Otherwise, $G$ has index 2 in $Z G$ (since $\lambda^{2} \cdot 1_{V} \in G$ ) and so either $r$ or $\lambda^{-1} r$ belongs to $G$. In either case, we see that $D$ contains a non-central element $s \in\left\{r, \lambda^{-1} r\right\}$ with age $(s)<1$ and $\left\langle s^{G}\right\rangle \leq D<G$, a contradiction. So $e=1$. Also $d>1$ as otherwise $H=G(1,1, n)=\mathrm{S}_{n}$ is reducible on $V$.

Conversely, we show that any c.r.g. $H=G(d, 1, n)=D: \mathrm{S}_{n}$ with $d>1$ yields a basic non-RT pair. Indeed, since $D<S L(V)$, for any non-central $x \in D$ we have $0<$ $\operatorname{age}(x) \in \mathbb{Z}$ and so age $(x) \geq 1$. Now consider any non-central $y \in H$ with age $(y)<1$ 
(such elements exist, for instance, one can take any transposition in $\mathrm{S}_{n}$ ). By our observation and by Lemma 5.1, $y$ induces a transposition, say $(1,2)$, on $\left\{V_{1}, \ldots, V_{n}\right\}$. We need to show that $K:=\left\langle y^{H}\right\rangle$ coincides with $H$. It is clear that $K D=H$. Next, for $\delta:=e^{2 \pi i / d}$ we have $z:=\operatorname{diag}\left(\delta, 1, \delta^{-1}, 1, \ldots, 1\right) \in D$ and $K \ni y z y^{-1} z^{-1}=\operatorname{diag}\left(\delta^{-1}, \delta, 1, \ldots, 1\right)$. It is now easy to see that the set of all $K$-conjugates of $y z y^{-1} z^{-1}$ generates $D$, and so $K \geq K D=H$.

3) From now on we will assume that we are in the situation of (i) but there is no finite subgroup $Z<Z(\mathcal{G})$ such that $Z G$ contains a complex reflection. By Lemma 2.2(ii), there is $\mu \in S^{1}$ of finite order such that age $(\mu g)=\operatorname{age}^{*}(g)<1$. Replacing $G$ by $\left\langle\mu \cdot 1_{V}\right\rangle \cdot G$ and $g$ by $\mu g$, we may (and will) assume that age $(g)<1$. By the conclusion of 1 ), we see that $D$ contains non-central elements $h$ with age $(h)<1$. By Lemma 5.4, $D$ is normalized by the monomial subgroup $S \cong \mathrm{S}_{n}$ of $G L(V)$ (that acts via permuting the basis vectors $\left.e_{1}, \ldots, e_{n}\right)$. In what follows we will freely conjugate elements of $D$ by elements of $S$.

Let $A=\left\{x_{1} \mid \exists \operatorname{diag}\left(x_{1}, \ldots\right) \in D\right\}$ be the finite subgroup of $S^{1}$ consisting of all the first diagonal entries of all the elements in $D$. Also, let

$$
\begin{aligned}
& B=\left\{x_{1} / x_{2} \mid \exists \operatorname{diag}\left(x_{1}, x_{2}, \ldots\right) \in D\right\}, \\
& C=\left\{\operatorname{diag}\left(z_{1}, \ldots, z_{n}\right) \mid z_{i} \in B, \prod_{i=1}^{n} z_{i}=1\right\} .
\end{aligned}
$$

Observe that $C \leq D$. Indeed, if $x=\operatorname{diag}\left(x_{1}, x_{2}, x_{3}, \ldots, x_{n}\right) \in D$, then some $S$-conjugate of $x$ equals $y=\operatorname{diag}\left(x_{2}, x_{1}, x_{3}, \ldots, x_{n}\right) \in D$, and so $D \ni x y^{-1}=\operatorname{diag}\left(\alpha, \alpha^{-1}, 1, \ldots, 1\right)$ with $\alpha=x_{1} / x_{2} \in B$. Conjugating $x y^{-1}$ suitably, we see that any diagonal matrix with spectrum $\left\{\alpha, \alpha^{-1}, 1, \ldots, 1\right\}$ (with counting multiplicities and $\alpha \in B$ ) belongs to $D$. Now any matrix $\operatorname{diag}\left(z_{1}, \ldots, z_{n}\right)$ with $z_{i} \in B$ and $\prod_{i=1}^{n} z_{i}=1$, is the product of $n-1$ diagonal matrices $\operatorname{diag}\left(z_{1}, z_{1}^{-1}, 1, \ldots, 1\right), \operatorname{diag}\left(1, z_{1} z_{2},\left(z_{1} z_{2}\right)^{-1}, 1, \ldots, 1\right), \ldots$, $\operatorname{diag}\left(1, \ldots, 1, z_{1} \ldots z_{n-1},\left(z_{1} \ldots z_{n-1}\right)^{-1}\right)$, all having spectrum of indicated shape, and so belongs to $D$.

4) Set $Z_{1}=\left\{z \cdot 1_{V} \mid z \in A\right\}<Z(\mathcal{G})$. We claim that $D Z_{1}=C Z_{1}$. Indeed, consider any $x=\operatorname{diag}\left(x_{1}, \ldots, x_{n}\right) \in D$. Conjugating $x$ suitably, we see that $y_{i}:=x_{i} / x_{n} \in B$ for $1 \leq i \leq n-1$ and $x_{n} \in A$. Now express $y:=\operatorname{diag}\left(y_{1}, y_{2}, \ldots, y_{n-1}, 1\right) \in D Z_{1}$ as $y=t_{1} t_{2} \ldots t_{n-1}$, where

$$
\begin{aligned}
& t_{1}=\operatorname{diag}\left(y_{1}, y_{1}^{-1}, 1, \ldots, 1\right), t_{2}=\operatorname{diag}\left(1, y_{1} y_{2},\left(y_{1} y_{2}\right)^{-1}, 1, \ldots, 1\right), \ldots, \\
& t_{n-2}=\operatorname{diag}\left(1, \ldots, 1, y_{1} \ldots y_{n-2},\left(y_{1} \ldots y_{n-2}\right)^{-1}, 1\right) \\
& t_{n-1}=\operatorname{diag}\left(1, \ldots, 1, y_{1} \ldots y_{n-1}, 1\right)
\end{aligned}
$$

Notice that $t_{1}, \ldots, t_{n-2} \in C$. If $y_{1} \ldots y_{n-1} \neq 1$, then obviously $t_{n-1} \in D Z_{1}<Z_{1} G$ is a complex reflection, a contradiction. It follows that $y_{1} \ldots y_{n-1}=1$. Now $y=t_{1} \ldots t_{n-2} \in$ $C$ and $x=x_{n} y \in C Z_{1}$ for all $x \in D$, and so $D Z_{1}=C Z_{1}$, as stated.

Let $|B|=b$. Denoting $\epsilon:=e^{2 \pi i / b}$, we have $v:=\operatorname{diag}\left(\epsilon, \epsilon, \ldots, \epsilon, \epsilon^{1-n}\right) \in C$ and $C Z_{1} \ni \epsilon^{-1} v=\operatorname{diag}\left(1, \ldots, 1, \epsilon^{-n}\right)$. In particular, if $\epsilon^{n} \neq 1$, then $\epsilon^{-1} v$ is a complex reflection, again a contradiction. Hence $\epsilon^{n}=1$ and so $b \mid n$. Now we turn our attention to the non-central element $h \in D$ with age $(h)<1$. Since $D Z_{1}=C Z_{1}$, we may write 
$h=\lambda c$ for some $\lambda \in S^{1}$ and $c \in C$. Clearly, age* $(c) \leq \operatorname{age}(h)<1$. By Lemma 2.2(ii) and its proof, there is some $\mu \in S^{1}$, where $\mu^{-1}$ is either 1 or one of the eigenvalues of $c$, such that age $(\mu c)=\operatorname{age}^{*}(c)<1$. By the construction of $C, \mu \in B$. Also, $\operatorname{det}(\mu c)=$ $\mu^{n} \operatorname{det}(c)=1$ as $b \mid n$. Now observe that $0 \leq \operatorname{age}(u) \in \mathbb{Z}$ for any $u \in S L(V)$. Applying this observation to $\mu c$, we see that age $(\mu c) \in \mathbb{Z}$. Since $0 \leq \operatorname{age}(\mu c)<1$, we must have age $(\mu c)=0$, and so $\mu c=1_{V}$. Thus $h=\lambda c$ is central, a contradiction.

Example 5.6. Let $2 \mid n \geq 6$. We exhibit an example of a finite irreducible (imprimitive) subgroup of $G L_{n}(\mathbb{C})$ which is generated by elements of age $=2 / 3$, but cannot be generated by complex reflections (up to scalars). First consider any $n \geq 5$. Pick a basis $\left(e_{1}, \ldots, e_{n}\right)$ of $V=\mathbb{C}^{n}$ and consider $G=\left\langle y_{1}, x_{2}, x_{3}, \ldots, x_{n-2}, z_{n-1}\right\rangle$, where

$$
\begin{aligned}
y_{1} & : e_{1} \leftrightarrow e_{2}, e_{j} \mapsto e_{j} \text { for } 3 \leq j \leq n-1, e_{n} \mapsto e^{\pi i / 3} e_{n}, \\
x_{i} & : e_{i} \leftrightarrow e_{i+1}, e_{j} \mapsto e_{j} \text { for } j \neq i, i+1, n, e_{n} \mapsto-e_{n}, \text { for } 1 \leq i \leq n-2, \\
z_{n-1} & : e_{n-1} \leftrightarrow e_{n}, e_{j} \mapsto e_{j} \text { for } 1 \leq j \leq n-3, e_{n-2} \mapsto-e_{n-2} .
\end{aligned}
$$

Also, consider the subgroup $G_{n}=\left\langle y_{1}^{3}=x_{1}, x_{2}, \ldots, x_{n-2}, z_{n-1}\right\rangle$ of $G$. Clearly, both $G$ and $G_{n}$ induce $\mathrm{S}_{n}$ while permuting the 1 -spaces $\left\langle e_{1}\right\rangle, \ldots,\left\langle e_{n}\right\rangle$. Next, we have $y_{1}^{2}=$ $\operatorname{diag}\left(1, \ldots, 1, e^{2 \pi i / 3}\right)$, and so $\left(y_{1}^{2}\right)^{G}$ generates a normal subgroup $E$ of order $3^{n}$ of $G$; furthermore, $G=E: G_{n}$. We claim that $G_{n}$ is an extension of $F=\left\{\operatorname{diag}\left(a_{1}, \ldots, a_{n}\right)\right\}$ $\left.a_{i}= \pm 1, \prod_{i=1}^{n} a_{i}=1\right\}$ by $S_{n}$. Indeed, it is clear that $G_{n}<S L(V)$, the normal subgroup $F_{1}$ of all diagonal elements of $G_{n}$ is contained in $F$, and $G_{n} / F_{1} \simeq \mathrm{S}_{n}$. We will obtain the claim, showing by induction on $n \geq 5$ that $F_{1}=F$. When $n=5$, a direct check using [GAP] shows that $\left|G_{5}\right|=2^{4} \cdot\left|\mathrm{S}_{5}\right|$ and so $F_{1}=F$. For the induction step, $\left\langle x_{2}, \ldots, x_{n-2}, z_{n-1}\right\rangle$ fixes $e_{1}$ and plays the role of $G_{n-1}$ while acting on $\left\langle e_{2}, \ldots, e_{n}\right\rangle$. By the induction hypothesis, $G_{n} \ni f:=\operatorname{diag}(1,1, \ldots, 1,-1,-1)$, whence $F_{1}=F$.

Next we show that $K:=\left\langle\left(y_{1}\right)^{G}\right\rangle$ equals $G$, and so $G$ is generated by elements of age $=2 / 3$. Clearly, $K$ induce $S_{n}$ while permuting the 1 -spaces $\left\langle e_{1}\right\rangle, \ldots,\left\langle e_{n}\right\rangle$. Also, $K \ni y_{1}^{2}$, and so $K>E$. Observe that $f=y_{1}^{3} \cdot z_{n-1} y_{1}^{3} z_{n-1}^{-1} \in K$, whence $K>F$ and so $K=E G_{n}=G$.

Finally, assuming $2 \mid n$, we show that any complex reflection in $Z(G L(V)) G$ is diagonal, and so $G$ cannot be generated by complex reflections (up to scalars). Assume the contrary: there is some $t \in G$ such that $\operatorname{Spec}(t)=\{\gamma, \delta, \ldots, \delta\}$ with $\gamma \neq \delta$ and $t$ is not diagonal. Since age* $(t)<1$, by Lemma 5.1 we may assume that $t \equiv x_{1}(\bmod E F)$, i.e. $t=x_{1} u$, with $u=\operatorname{diag}\left(u_{1}, \ldots, u_{n}\right), u_{j}=\epsilon^{m_{j}}$ for some $m_{j} \in \mathbb{Z}$ and $\epsilon:=e^{\pi i / 3}$, and $\sum_{j=1}^{n} m_{j} \in 2 \mathbb{Z}$. Since $\operatorname{Spec}(t)=\left\{\sqrt{u_{1} u_{2}},-\sqrt{u_{1} u_{2}}, u_{3}, \ldots, u_{n-1},-u_{n}\right\}$, we must have $-\gamma=\delta=\epsilon^{k}$ for some $k \in \mathbb{Z}$. Now

$$
-1=\left(-\delta^{n}\right)^{3}=(\operatorname{det}(t))^{3}=(\operatorname{det}(u))^{3}=\left(\prod_{j=1}^{n} u_{j}\right)^{3}=\epsilon^{3 \sum_{j=1}^{n} m_{j}}=1
$$

(since $2 \mid n$ ), a contradiction.

Finally, we prove an analogue of Theorem 5.5(ii) for age $\leq 1$ : 
Theorem 5.7. Let $G<G L(V)$ be a finite imprimitive, irreducible subgroup. Assume $G$ contains non-central elements $g$ with age $(g) \leq 1$, and $Z G=Z \cdot\left\langle g^{G}\right\rangle$ for any such element $g$, where $Z:=Z(G L(V))$. Then $\operatorname{dim}(V) \leq 8$.

Proof. Assume the contrary: $\operatorname{dim}(V) \geq 9$ for such a group $G$. Clearly, $G$ satisfies the set-up ( $)$. Hence $G$ satisfies one of the conclusions (i) and (ii) of Lemma 5.2.

1) Suppose the conclusion (i) of Lemma 5.2 holds. Since $n=\operatorname{dim}(V) \geq 9$, we have $\pi(G) \geq \mathrm{A}_{n}$. Let $D=\operatorname{Ker}(\pi)$ be the subgroup of all diagonal elements of $G$, in a basis $\left(e_{1}, \ldots, e_{n}\right)$ such that $V_{i}=\left\langle e_{i}\right\rangle$.

First we consider the case $D \not Z Z$. Then we may assume $D \ni x=\operatorname{diag}\left(x_{1}, \ldots, x_{n}\right)$ with $x_{1} \neq x_{2}$. Choosing $s \in G$ with $\pi(s)=(1,2,3)$, we get

$$
\begin{aligned}
G \ni y=[s, x] & =\operatorname{diag}\left(x_{3} / x_{1}, x_{1} / x_{2}, x_{2} / x_{3}, 1, \ldots, 1\right) \\
& =\operatorname{diag}\left(e^{2 \pi i a}, e^{2 \pi i b}, e^{2 \pi i c}, 1, \ldots 1\right),
\end{aligned}
$$

where $0 \leq a, c<1,0<b<1$, and $a+b+c \in \mathbb{Z}$. It follows that either $a+b+c=1$, in which case age $(y)=1$, or $a+b+c=2$, in which case age $\left(y^{-1}\right)=1$. In either case, we have found a diagonal non-central element $z$ with age $(z)=1$. It is clear that $Z\left\langle z^{G}\right\rangle$ is diagonal and so cannot contain $G$, a contradiction.

We have shown that $D \leq Z$ and so $V$ yields an irreducible projective representation of degree $n \geq 9$ of $\mathrm{S}_{n}$ or $\mathrm{A}_{n}$, which is impossible by degree consideration.

2) Now we assume that the conclusion (ii) of Lemma 5.2 holds: $G=D: \mathrm{S}_{n}$, with $n \geq 3, D<G L_{2}(\mathbb{C})^{n}$ and the action of $\mathrm{S}_{n}$ described in (10) for a fixed basis $\left(u_{i}, v_{i}\right)$ of each $V_{i}$. Let $A=\left\{x_{1} \mid \exists \operatorname{diag}\left(x_{1}, \ldots\right) \in D\right\}$ be the finite subgroup of $G L_{2}(\mathbb{C})$ afforded by the action of $D$ on $V_{1}$, with respect to the basis $\left(u_{1}, v_{1}\right)$. Also, let

$$
\begin{aligned}
& B=\left\{x_{1} x_{2}^{-1} \mid \exists \operatorname{diag}\left(x_{1}, x_{2}, \ldots\right) \in D\right\}, \\
& C=\left\{\operatorname{diag}\left(z_{1}, \ldots, z_{n}\right) \mid z_{i} \in B, \prod_{i=1}^{n} z_{i}=I\right\},
\end{aligned}
$$

where $I$ denotes the identity $2 \times 2$-matrix. Note that, by their definition, $B$ and $C$ are finite sets. Consider any $a \in B$. Then we can find $x=\operatorname{diag}\left(x_{1}, x_{2}, x_{3}, \ldots, x_{n}\right) \in D$ with $a=x_{1} x_{2}^{-1}$, and some conjugate $y=\operatorname{diag}\left(x_{2}, x_{1}, x_{3}, \ldots, x_{n}\right) \in D$ of $x$, whence $D \ni x y^{-1}=\alpha:=\operatorname{diag}\left(a, a^{-1}, I, \ldots, I\right)$. Conjugating $\alpha$ suitably, we see that any matrix $\operatorname{diag}\left(I, \ldots, I, a, a^{-1}, I, \ldots, I\right)$ belongs to $D$. Similarly, if $b \in B$, then $\beta:=$ $\operatorname{diag}\left(b, b^{-1}, I, \ldots, I\right) \in D$, and $D \ni \alpha \beta=\operatorname{diag}\left(a b, a^{-1} b^{-1}, I, \ldots, I\right)$. Conjugating the latter element suitably, we see that $D \ni \operatorname{diag}\left(a b, I, a^{-1} b^{-1}, I, \ldots, I\right)$ and so $a b \in B$. Thus $B$ is closed under multiplication and so it is a group by finiteness. By the above observation applied to $a b, \gamma=\operatorname{diag}\left(a b,(a b)^{-1}, I, \ldots, I\right)$ belongs to $D$, and so does $\delta:=\gamma^{-1} \alpha \beta=\operatorname{diag}(I,[a, b], I, \ldots, I)$. Note that, since $[a, b] \in S L_{2}(\mathbb{C})$ (and has finite order $|\delta|)$, we have either age $([a, b])=1$, or $[a, b]=I$. In the former case, age $(\delta)=1$ and $Z\left\langle\delta^{G}\right\rangle \leq Z D \nsupseteq G$, a contradiction. Hence, $[a, b]=I$ for all $a, b \in B$, i.e. $B$ is an abelian group. This in turn implies that $C$ is a subgroup of $D$.

Observe that $A$ normalizes $B$. (Indeed, for any $x_{1} \in A$ and $b \in B$, there is some $x=\operatorname{diag}\left(x_{1}, x_{2}, \ldots, x_{n}\right) \in D$ and $u=\operatorname{diag}\left(b, I, b^{-1}, I, \ldots, I\right) \in D$. Hence $D \ni x u$ 
$=\operatorname{diag}\left(x_{1} b, x_{2}, \ldots\right)$ and so $B \ni x_{1} b\left(x_{2}\right)^{-1}=x_{1} b x_{1}^{-1} \cdot x_{1} x_{2}^{-1}$. But $x_{1} x_{2}^{-1} \in B$, hence $x_{1} b x_{1}^{-1} \in B$ as stated.) Since $D \leq A \times \cdots \times A$ and $G=D: \mathrm{S}_{n}$, we see that $C \triangleleft G$. In fact, we claim that $\left[\mathrm{S}_{n}, D\right] \leq C$. To prove this, let us identify the action of $\sigma \in \mathrm{S}_{n}$ with its action on $\left\{u_{1}, \ldots, u_{n}\right\}$ and on $\left\{v_{1}, \ldots, v_{n}\right\}$ (cf. (10)). Then for any $x=\operatorname{diag}\left(x_{1}, x_{2}, \ldots, x_{n}\right) \in D$ we have $\sigma^{-1} x \sigma x^{-1}=\operatorname{diag}\left(b_{1}, \ldots, b_{n}\right)$, where $b_{i}=x_{\sigma(i)} x_{i}^{-1} \in B$. Now choose $\sigma=(j, j+1)$ for $1 \leq j \leq n-1$. Then we get $b_{i}=I$ for $i \neq j, j+1$, and $b_{j} b_{j+1}=x_{j+1} x_{j}^{-1} \cdot x_{j} x_{j+1}^{-1}=I$. It follows that $\sigma^{-1} x \sigma x^{-1} \in C$, and so $\sigma^{-1}(x C) \sigma=x C$ in $D / C$ for all $\sigma=(j, j+1)$. Consequently, $\sigma^{-1}(x C) \sigma=x C$ in $D / C$ for all $\sigma \in \mathrm{S}_{n}$, as stated.

We have shown that $C \triangleleft G=D \mathrm{~S}_{n}$ and $\left[D, \mathrm{~S}_{n}\right] \leq C$. Hence the subgroup $C \mathrm{~S}_{n}$ is normal in $G$. Recall that $g_{1}=(1,2)$ has age $=1$ and $g_{1} \in \mathrm{S}_{n}$. By our assumptions, $Z G=Z\left\langle\left(g_{1}\right)^{G}\right\rangle \leq K:=Z C S_{n}$. It follows that $Z G=K$. Let $\Phi$, resp. $\Phi_{1}$, denote the representation of $Z G$ on $V$, resp. of $G_{1}:=\operatorname{Stab}_{Z G}\left(V_{1}\right)$ on $V_{1}$. Then $G_{1}=Z C S_{n-1}$, where $S_{n-1}$ is acting trivially on $V_{1}$. Hence $\Phi_{1}\left(G_{1}\right)=\Phi_{1}(Z C)=\mathbb{C}^{\times} B$ is abelian. But $\operatorname{dim}\left(V_{1}\right)=2$, so $\Phi_{1}$ is reducible. Since $\Phi=\operatorname{Ind}_{G_{1}}^{Z G}\left(\Phi_{1}\right)$, we conclude that $Z G$ is reducible on $V$, a contradiction.

\subsection{Extraspecial case}

Here, $G \leq N:=N_{\mathcal{G}}(E)$ for some $p$-group $E$ of extraspecial type. By [GT1, Lemma 2.4], either $|\chi(g)|=0$, or $|\chi(g)|^{2}=\left|C_{E / Z(E)}(g)\right|$. It follows that $\Delta(g) \geq p^{m}(1-1 / \sqrt{p})$. Recall that $C=9.111$ in the set-up $(\boldsymbol{A})$. Hence $\operatorname{dim}(V)=p^{m} \leq 9.111(1+1 / \sqrt{2})$ and so $p^{m} \leq 13$. Since we are assuming $\operatorname{dim}(V) \geq 4$, we need to consider the following cases.

- $\operatorname{dim}(V)=p \geq 11$. Then $\Delta(g) \geq 11-\sqrt{11}>7.68$, and so age* $(g)>1$.

- $\operatorname{dim}(V)=p^{m}=9$. Here, $\Delta(g) \geq 9-3 \sqrt{3}$; moreover, if $|\chi(g)| \leq 3$ then $\Delta(g) \geq 6$. Thus we may assume that $|\chi(g)|=3 \sqrt{3}$. Next, $E=3_{+}^{1+4}$, and the character table of $N=Z(\mathcal{G}) E: S p_{4}(3)$ has been constructed explicitly by T. Breuer. Now one can verify directly that $N / Z(N)$ contains two classes of elements with $|\chi(g)|=3 \sqrt{3}$; any such element acts on $E / Z(E)=\mathbb{F}_{3}^{4}$ as a symplectic transvection. One of these classes has age ${ }^{*}=1$; the other class and all remaining non-central elements in $G$ have age* $>1$.

- $\operatorname{dim}(V)=p^{m}=8$. Here, $\Delta(g) \geq 8-4 \sqrt{2}$ and $E=C_{4} * 2_{+}^{1+6}$. The character table of $N=Z(\mathcal{G}) E \cdot S p_{6}(2)$ has been constructed explicitly by Breuer. In particular, $\operatorname{Irr}(N)$ contains two, complex-conjugate, characters of degree 8 . Hence it suffices to consider one of these two characters and the classes of $g$ with $|\chi(g)| \geq 4$. Now one can verify directly that $N / Z(N)$ contains three conjugacy classes of elements $g$ with age* $(g)=1$; their spectra are listed in items (b1) and (c6) of Remark 3.9. In all other cases, age* $(g)>1$ by Lemmas 2.20 and 2.21 .

- $\operatorname{dim}(V)=p^{m}=7$. Here, $\Delta(g) \geq 7-\sqrt{7}$; moreover, if $|\chi(g)| \leq 1$ then $\Delta(g) \geq 6$. Thus we may assume that $|\chi(g)|=\sqrt{7}$. Next, $E=7_{+}^{1+2}$, and the character table of $N=Z(\mathcal{G}) E: S p_{2}(7)$ has been constructed explicitly by Breuer. In particular, $\operatorname{Irr}(N)$ contains seven characters of degree 7 , with exactly six being faithful on $E$, each of which is uniquely determined by its central character. Hence it suffices to consider one of these six characters. Now one can verify directly that in the cases where $|\chi(g)|=\sqrt{7}$, the 
smallest arc of $S^{1}$ that contains all eigenvalues of $g$ has length $\delta \geq \pi$, and so age* $(g)>1$ by Corollary 2.18 .

- $\operatorname{dim}(V)=p^{m}=5$. Here, $\Delta(g) \geq 5-\sqrt{5}$ and $E=5_{+}^{1+2}$. The character table of $N=Z(\mathcal{G}) E: S p_{2}(5)$ has been constructed explicitly by Breuer. In particular, $\operatorname{Irr}(N)$ contains five characters of degree 5 , with exactly four being faithful on $E$, each of which is uniquely determined by its central character. Hence it suffices to consider one of these four characters. Now one can verify directly that $N / Z(N)$ contains three conjugacy classes of elements $g$ with age* $(g)=1$; their spectra are listed in items (b1), (c4), and (c5) of Remark 3.9. In all other cases, age* $(g)>1$ by Corollary 2.18 (with $\delta \geq 6 \pi / 5$ ).

- $\operatorname{dim}(V)=p^{m}=4$. Here, $\Delta(g) \geq 4-2 \sqrt{2}$ and $E=C_{4} * 2_{+}^{1+4}$. The character table of $N=Z(\mathcal{G}) E \cdot S p_{4}(2)$ has been constructed explicitly by Breuer. In particular, $\operatorname{Irr}(N)$ contains two pairs $(\alpha, \bar{\alpha})$ and $(\beta, \bar{\beta})$ of complex-conjugate characters of degree 4; furthermore, $\beta$ can be obtained from $\alpha$ by tensoring with the sign character of $S p_{4}(2) \simeq \mathrm{S}_{6}$. Hence we may assume that $\chi=\alpha$. Now one can verify directly that $N / Z(N)$ contains three conjugacy classes of elements $g$ with $0<$ age* $^{*}(g)<1$ and spectra as listed in items (a1), (a4), and (c1) of Remark 3.9. Fixing an isomorphism between $S_{4}(2)$ and $\mathrm{S}_{6}$, we may assume that these three classes project onto the classes of $(1,2)$, resp. $(1,2,3),(1,2)(3,4)(5,6)$, in $\mathrm{S}_{6} . N / Z(N)$ also contains several classes of elements $g$ with age* $(g)=1$ and spectra as listed in items (b1), (b2), (b4), (c2), and (c3) of Remark 3.9. In all other cases, age* $(g)>1$. Now we show that $N$ contains a subgroup $M$ leading to a basic non-RT pair not of reflection type.

Lemma 5.8. There is a subgroup $M=C_{3} \times\left(C_{4} * 2_{+}^{1+4}\right) \cdot \mathrm{A}_{6}<G L\left(\mathbb{C}^{4}\right)$ which gives rise to a basic non-RT pair not of reflection type.

Proof. Since $M \triangleright E$, the subgroup $M$ acts irreducibly on $V=\mathbb{C}^{4}$. Notice that $Z(\mathcal{G}) M=$ $Z(\mathcal{G})[N, N]$ has index 2 in $Z(\mathcal{G}) N$. By the above analysis, all non-central elements $g \in M$ with age* $(g)<1$ in $M$ are $[N, N]$-conjugate to an element $g$ with spectrum $\left(e^{2 \pi i / 3}, e^{2 \pi i / 3}, 1,1\right)$ which corresponds to the class of $(1,2,3)$ in $\mathrm{A}_{6}$. In fact one can choose such an element $g$ in $C_{3} \times 2 \mathrm{~A}_{6}<M$ with age $(g)<1$. We have shown that $g^{M}=\{h \in M \backslash Z(M) \mid \operatorname{age}(h)<1\}$ and that $M$ contains no complex reflection. It remains to show that $\left\langle g^{M}\right\rangle=M$.

Denote $C:=C_{3} \times C_{4}<Z(\mathcal{G}), E:=C_{4} * 2_{+}^{1+4}, O=E C, M_{1}:=E \cdot \mathrm{A}_{6}<M=$ $C_{3} \times M_{1}$, and $K:=\left\langle g^{M}\right\rangle$. Since $(1,2,3)^{\mathrm{A}_{6}}$ generates $\mathrm{A}_{6}$, we must have $K O=M$. Next, since $O / C$ is the unique minimal normal subgroup of $M / C$, we see that $K C \geq O$ and so $K C=K O=M$. Observe that $[M, M]=\left[M_{1}, M_{1}\right]=M_{1}$. (Indeed, it is easy to check that $\left[M_{1}, M_{1}\right]$ contains $2_{+}^{1+4} \cdot \mathrm{A}_{6}$ and so it has index at most 2 in $M_{1}$. But $M_{1}$ is a normal subgroup of index 2 in $E \cdot \mathrm{S}_{6}$, and one can check that $E \cdot \mathrm{S}_{6}$ has only two linear characters. It follows that $\left[M_{1}, M_{1}\right]=M_{1}$.) Now we have $K \geq[K, K]=[K C, K C]=$ $[M, M]=M_{1}$. Also, $M_{1}$ is a perfect subgroup of $G L(V)$, whence $M_{1}<S L(V)$. But $\operatorname{det}(g)=e^{4 \pi i / 3} \neq 1$, so $M \geq K>M_{1}$. Since $M / M_{1} \cong C_{3}$, we conclude that $K=M$.

We will need the following complement to Theorem 1.3: 
Theorem 5.9. Let $G<G L(V)$ be a finite, irreducible, primitive, tensor indecomposable subgroup and let $g \in G \backslash Z(G)$. If the $G$-module $V$ is tensor induced, assume in addition that $g$ acts non-trivially on the set of tensor factors of $V$.

(i) If $\operatorname{dim}(V)=2$, then age* $(g) \geq 1 / 5$.

(ii) If $\operatorname{dim}(V)=3$ or 4 , then age* $(g) \geq 1 / 3$.

(iii) If $\operatorname{dim}(V)>4$, then age* $(g) \geq 1 / 2$.

Proof. 1) First we consider the case $V$ is tensor induced; in particular, $d:=\operatorname{dim}(V)=a^{m}$ for some integers $a, m \geq 2$, and $\Delta(g) \geq d(1-1 / a)$ by Lemma 2.25 . Now if $d=4$, then $\Delta(g) \geq 2$ and so age* $(g) \geq 4 /(2.9 \pi)>0.43$ by Proposition 2.17(iii). If $d \geq 5$, then $\Delta(g) \geq 4$ and so age* $(g) \geq 8 /(2.9 \pi)>0.86$ again by Proposition 2.17(iii) (in fact, age* $(g)>1$ unless $d=8$ ). From now on we may assume that $V$ is not tensor induced.

2) Consider the case $d=2$ and assume that age $(g)<1 / 5$. Then we may write $\operatorname{Spec}(g)=\left\{1, e^{i \alpha}\right\}$ with $0<\alpha<2 \pi / 5$. It follows that $|\operatorname{Tr}(g)|=2 \cos (\alpha / 2)>$ $2 \cos (\pi / 5)=(1+\sqrt{5}) / 2$ and so $\Delta(g)<(3-\sqrt{5}) / 2$, contradicting Theorem 1.3(i).

Next assume $d=3$ and age* $(g)<1 / 3$. Then we may write $\operatorname{Spec}(g)=\left\{1, e^{i \alpha}, e^{i \beta}\right\}$ with $0 \leq \alpha \leq \beta \leq \alpha+\beta<2 \pi / 3$; in particular,

$$
|\operatorname{Tr}(g)|^{2}=3+2 \cos (\beta)+4 \cos (\beta / 2) \cos (\beta / 2-\alpha) .
$$

Now $\cos (\beta)>-1 / 2$ and $\cos (\beta / 2)>1 / 2$ as $0 \leq \beta<2 \pi / 3$. Also, $\cos (\beta / 2-\alpha)>1 / 2$, since $-\pi / 3<-\alpha / 2 \leq \beta / 2-\alpha \leq \beta / 2<\pi / 3$. It follows that $|\operatorname{Tr}(g)|^{2}>3-1+1=3$ and so $\Delta(g)<3-\sqrt{3}$, contradicting Theorem 1.3(ii).

3) Now we may assume that $d \geq 4$. If we are in the extraspecial case, then age* $(g) \geq$ $1 / 2$ by the results of $\S 5.2$. Otherwise, by [GT3, Proposition 2.8] we may apply Theorem 3.8.

Note that the lower bounds given in Theorem 5.9 are best possible: cf. the examples of $S L_{2}(5)<G L_{2}(\mathbb{C}), 3_{+}^{1+2}: S L_{2}(3)<G L_{3}(\mathbb{C})$, and Table I for examples in dimensions $\geq 4$.

\subsection{Tensor decomposable case}

Lemma 5.10. In the set-up (\$), assume that the $G$-module $V$ is primitive and tensor decomposable. Then $d:=\operatorname{dim}(V) \leq 10$.

Proof. Write $V=V_{1} \otimes \cdots \otimes V_{m}$, where $V_{i}$ are irreducible, primitive, tensor indecomposable $G$-modules of dimension $\geq 2$, and $m \geq 2$.

1) Consider the case where $\operatorname{dim}\left(V_{i}\right) \geq 3$, say for $i=1$, and set $W:=V_{2} \otimes \cdots \otimes V_{m}$. Then we can find $g \in \mathcal{X}$ such that $\left.g\right|_{V_{1}}$ is non-scalar. In case the $G$-module $V_{1}$ is tensor induced, among such elements $g$ we can find one that acts non-trivially on the set of tensor factors of $V_{1}$ (as otherwise the $G$-module $V_{1}$ would be tensor decomposable). By Theorem 5.9, age* $\left(\left.g\right|_{V_{1}}\right) \geq 1 / 3$. By Lemma $2.2\left(\right.$ iv), $1 \geq \operatorname{age}^{*}(g) \geq \operatorname{dim}(W) \cdot \operatorname{age}^{*}\left(\left.g\right|_{V_{1}}\right)$. It follows that $\operatorname{dim}(W) \leq 3$ and so $m=2$. Again, we can find $h \in \mathcal{X}$ such that $\left.h\right|_{V_{2}}$ is non-scalar. Notice that the $G$-module $V_{2}$ is not tensor induced. 
Assume $\operatorname{dim}\left(V_{2}\right)=3$. Then age* $\left(\left.h\right|_{V_{2}}\right) \geq 1 / 3$ by Theorem 5.9(ii). Now by Lemma 2.2(iv), $1 \geq \operatorname{age}(h) \geq \operatorname{dim}\left(V_{1}\right) \cdot \operatorname{age}\left(\left.h\right|_{V_{2}}\right)$. It follows that $\operatorname{dim}\left(V_{1}\right) \leq 3$ and so $d \leq 9$.

Assume $\operatorname{dim}\left(V_{2}\right)=2$. Then age* $\left(\left.h\right|_{V_{2}}\right) \geq 1 / 5$ by Theorem 5.9(i). Now by Lemma 2.2(iv), $1 \geq \operatorname{age}^{*}(h) \geq \operatorname{dim}\left(V_{1}\right) \cdot \operatorname{age}\left(\left.h\right|_{V_{2}}\right)$. It follows that $\operatorname{dim}\left(V_{1}\right) \leq 5$ and so $d \leq 10$.

2) Now assume that $\operatorname{dim}\left(V_{i}\right)=2$ for all $i$; in particular, the $G$-module $V_{i}$ is not tensor induced. Then we can find $g \in \mathcal{X}$ such that $\left.g\right|_{V_{1}}$ is non-scalar. By Theorem 5.9(i), age* $\left(\left.g\right|_{V_{1}}\right) \geq 1 / 5$. It now follows from Lemma 2.2 (iv) that $1 \geq \operatorname{age}(g) \geq\left(d / \operatorname{dim}\left(V_{1}\right)\right)$. age* $\left(\left.g\right|_{V_{1}}\right), d / \operatorname{dim}\left(V_{1}\right) \leq 5$, and so $d \leq 10$. In fact, $d=4$ or 8 in this case.

The example of $\left(C_{5} \times S L_{2}(5)\right) *\left(C_{2} \times S U_{4}(2)\right)$ acting on $\mathbb{C}^{2} \otimes \mathbb{C}^{5}$ shows that the bound 10 in Lemma 5.10 is best possible.

Lemma 5.11. In the set-up $(\mathbf{\bullet})$, assume that the $G$-module $V$ is primitive and tensor decomposable and that $G$ contains a non-central element $g$ with age $(g)<1$. Then $\operatorname{dim}(V) \leq 8$.

Proof. Assume the contrary: $d:=\operatorname{dim}(V) \geq 9$. Notice that $d \leq 10$ by Lemma 5.10 . It follows that $d=9$ or 10 , and $V=A \otimes B$, where $A$ and $B$ are irreducible, primitive, tensor indecomposable, not tensor induced, $G$-modules of dimension $>1$. Since $g \notin Z(G)$, we may assume that $\left.g\right|_{A}$ is not scalar.

Assume $\operatorname{dim}(A)=3$ (and so $\operatorname{dim}(B)=3$ ). Then age* $\left(\left.g\right|_{A}\right) \geq 1 / 3$ by Theorem 5.9(ii), and so age* $(g) \geq \operatorname{dim}(B) \cdot \operatorname{age}^{*}\left(\left.g\right|_{A}\right) \geq 1$ by Lemma 2.2(iv), a contradiction. Thus $\operatorname{dim}(A)=2$ or 5 . Assume $\operatorname{dim}(A)=2$ (and so $\operatorname{dim}(B)=5$ ). Then age* $\left(\left.g\right|_{A}\right) \geq$ $1 / 5$ by Theorem 5.9(i). Again by Lemma 2.2(iv), age* $(g) \geq \operatorname{dim}(B) \cdot \operatorname{age}\left(\left.g\right|_{A}\right) \geq 1$, a contradiction. Finally, let $\operatorname{dim}(A)=5$ (and $\operatorname{so} \operatorname{dim}(B)=2$ ). Then age* $\left(\left.g\right|_{A}\right) \geq 1 / 2$ by Theorem 5.9(iii), and so age* $(g) \geq \operatorname{dim}(B) \cdot \operatorname{age}^{*}\left(\left.g\right|_{A}\right) \geq 1$, again a contradiction.

The example of $\left(C_{5} \times S L_{2}(5)\right) *\left(C_{3} \times S p_{4}(3)\right)$ acting on $\mathbb{C}^{2} \otimes \mathbb{C}^{4}$ shows that the bound 8 in Lemma 5.11 is best possible.

Lemma 5.12. Let $g=A \otimes B$, where $A \in G<G L_{m}(\mathbb{C}), B \in H<G L_{n}(\mathbb{C})$, with $m \geq 3$ and $n \geq 2$. Assume that $G$ and $H$ are finite primitive irreducible subgroups, and that $A, B$ are non-scalar. Then age* $(g)>1$.

Proof. 1) Assume the contrary: age* $(g) \leq 1$. By a well-known result of Blichfeldt (cf. [D]), the smallest arc that contains all eigenvalues of any non-central element in any finite, primitive, irreducible linear group has length $\geq \pi / 3$. Thus $\alpha, \beta \geq 1 / 6$, where $2 \pi \alpha$, resp. $2 \pi \beta$, is the length of such smallest arc for $A$, resp. for $B$. In particular, $\operatorname{age}^{*}(A)$, age* $(B) \geq 1 / 6$. On the other hand, age* $(A) \leq 1 / n \leq 1 / 2$ and age* $(B) \leq$ $1 / m \leq 1 / 3$ by Lemma 2.2(iv), whence $\alpha \leq 1 / 2$ and $\beta \leq 1 / 3$. By Lemma 2.2(ii), we can multiply $g$ by a suitable scalar and assume that age $(g) \leq 1$. Multiplying $B$ by a suitable $\mu \in S^{1}$ and $A$ by $\mu^{-1}$, we may assume that $\operatorname{Spec}(B) \ni 1, e^{2 \pi i \beta}$, and all other eigenvalues of $B$ belong to the $\operatorname{arc}\left[1, e^{2 \pi i \beta}\right]$ of $S^{1}$. Write $A=\operatorname{diag}\left(e^{2 \pi i \alpha_{1}}, \ldots, e^{2 \pi i \alpha_{m}}\right)$ with $0 \leq \alpha_{j}<1$. 
2) Here we consider the case $n \geq 3$. Then $B$ has a third eigenvalue $e^{2 \pi i \delta}$ with $0 \leq$ $\delta \leq \beta$. For $0<\gamma \leq 1$, observe that

$$
\operatorname{age}\left(e^{-2 \pi i \gamma} B\right) \geq \begin{cases}1+\beta+\delta-3 \gamma \geq 1+\beta-2 \delta, & 0<\gamma \leq \delta, \\ 2+\beta+\delta-3 \gamma \geq 2(1-\beta)>1, & \delta<\gamma \leq \beta, \\ \beta+\delta+3(1-\gamma) \geq \beta+\delta, & \beta<\gamma \leq 1\end{cases}
$$

We will apply this observation to $\gamma=\gamma_{j}:=1-\alpha_{j}$. Since $1 \geq \operatorname{age}(A \otimes B)=$ $\sum_{j=1}^{m}$ age $\left(e^{-2 \pi i \gamma_{j}} B\right)$, we see that all $\gamma_{j}$ must belong to $(\beta, 1]$, and

$$
1 \geq \operatorname{age}(A \otimes B) \geq \sum_{j=1}^{m}\left(\beta+\delta+3\left(1-\gamma_{j}\right)\right) \geq m \beta+3 \sum_{j=1}^{m} \alpha_{j}=m \beta+3 \operatorname{age}(A) .
$$

Recall $m \geq 3, \beta \geq 1 / 6$, and age $(A) \geq 1 / 6$. It follows that $m=3$ and age $(A)=1 / 6$. The last equality however contradicts Theorem 5.9(ii) applied to the element $A$ of $G$.

3 ) Now we let $n=2$. For $0<\gamma \leq 1$, we have

$$
\operatorname{age}\left(e^{-2 \pi i \gamma} B\right)= \begin{cases}1+\beta-2 \gamma \geq 1-\beta, & 0<\gamma \leq \beta \\ 2+\beta-2 \gamma \geq \beta, & \beta<\gamma \leq 1\end{cases}
$$

We will again apply this observation to $\gamma=\gamma_{j}:=1-\alpha_{j}$ to estimate age $(A \otimes B)=$ $\sum_{j=1}^{m}$ age $\left(e^{-2 \pi i \gamma_{j}} B\right)$. If at least one $\gamma_{j}$ belongs to $(0, \beta]$, then age $(g) \geq 1-\beta+(m-1) \beta$ $\geq 1+(m-2) \beta>1$, a contradiction. Hence, all $\gamma_{j}$ belong to $(\beta, 1]$, and so

$$
1 \geq \operatorname{age}(A \otimes B)=\sum_{j=1}^{m}\left(\beta+2\left(1-\gamma_{j}\right)\right)=m \beta+2 \sum_{j=1}^{m} \alpha_{j}=m \beta+2 \operatorname{age}(A) .
$$

Recall that $m \geq 3$, age $(A) \geq 1 / 6$, and $\beta=\operatorname{age}(B) \geq 1 / 5$ by Theorem 5.9(i) applied to the element $B$ of $H$. It follows that $m \leq(1-2 / 6) /(1 / 5)$ and so $m=3$. But in this case, Theorem 5.9(ii) applied to the element $A$ of $G$ implies that age $(A) \geq 1 / 3$ and so age $(A \otimes B) \geq 3 / 5+2 / 3>1$.

Corollary 5.13. Let $G<G L(V)$ be a finite primitive irreducible subgroup. Assume that $\operatorname{dim}(V) \geq 5$ and that the $G$-module $V$ is tensor decomposable. Then, for any $g \in G$ with age* $(g) \leq 1, Z G \neq Z\left\langle g^{G}\right\rangle$, where $Z:=Z(G L(V))$.

Proof. Write $V=A \otimes B$ for some $G$-modules $A, B$ of dimension $>1$. Then $G$ is irreducible and primitive on both $A$ and $B$. Now consider any $g \in G$ with age* $(g) \leq 1$. By Lemma 5.12, $g$ must act scalarly on $A$ or on $B$, say on $A$. In this case, $H:=\left\langle g^{G}\right\rangle$ also acts scalarly on $A$ and so $Z G \neq Z H$ by irreducibility of $G$ on $A$.

\subsection{Tensor induced case}

Proposition 5.14. In the set-up (A), assume that the $G$-module $V$ is primitive, tensor indecomposable, but tensor induced. Then $\operatorname{dim}(V)=4$ or 8 . Moreover, if $\operatorname{dim}(V)=8$, then $G$ cannot be generated by its elements $h$ with age* $(h)<1$ (modulo scalars). 
Proof. 1) By the assumptions, there is a tensor decomposition $V=V_{1}^{\otimes m}$ with $a:=$ $\operatorname{dim}\left(V_{1}\right)>1$ and $m>1$ such that $G<G L\left(V_{1}\right)^{\otimes m}: \mathrm{S}_{m}$, and there is some $g \in \mathcal{X}$ such that $0<$ age $^{*}(g) \leq 1$ and $g$ acts non-trivially on the $m$ tensor factors of $V$. By Lemma $2.25,4.556>\Delta(g)=\operatorname{dim}(V)-|\operatorname{Tr}(g)| \geq a^{m-1}(a-1)$. It follows that $a=2$ and $m=2$ or 3 .

From now on we assume that $(a, m)=(2,3)$, i.e. $V=V_{1} \otimes V_{2} \otimes V_{3}$ and $\operatorname{dim}\left(V_{i}\right)$ $=2$. Then $g$ must project onto a 2-cycle of $S_{3}$, as otherwise by Lemma 2.25, $\Delta(g) \geq$ $8-2=6$ and so age $(g)>1$. Without loss we may assume that $g=A \otimes B$, with $A<G L\left(V_{1}\right)^{\otimes 2}: \mathrm{S}_{2}$ permuting the two tensor factors $V_{1}$ and $V_{2}$, and $B \in G L\left(V_{3}\right)$, and that $g$ has finite order: $g^{N}=I_{8}$ for some integer $N>1$. (Here we let $I_{n}$ denote the identity $n \times n$-matrix.) It follows that $A^{N} \otimes B^{N}=I_{8}=I_{4} \otimes I_{2}$. By (the first sentence of) the proof of Lemma 2.24, we can multiply $A$ by a suitable $\lambda \in \mathbb{C}^{\times}$(and $B$ by $\lambda^{-1}$ ) such that $A^{N}=I_{4}$ and $B^{N}=I_{2}$.

2) Here we show that age* $(A) \geq 1 / 2$ and age* $(g) \geq 1$. By our assumptions, there are some bases $\left(e_{1}, e_{2}\right)$ of $V_{1}$ and $\left(f_{1}, f_{2}\right)$ of $V_{2}$, and matrices $X, Y \in G L_{2}(\mathbb{C})$ such that, in the basis $\left(e_{1} \otimes f_{1}, e_{2} \otimes f_{1}, e_{1} \otimes f_{2}, e_{2} \otimes f_{2}\right)$ of $V_{1} \otimes V_{2}, A=\mathbf{j}(X \otimes Y)$, where $\mathbf{j}: e_{i} \otimes f_{j} \mapsto e_{j} \otimes f_{i}$. Now direct computation shows that

$$
\operatorname{det}(A-t I)=t^{4}-\operatorname{Tr}(X Y) \cdot t^{3}+\operatorname{Tr}(X Y) \cdot \operatorname{det}(X Y) \cdot t-\operatorname{det}(X Y)^{2} .
$$

In particular, writing $\operatorname{Spec}(X Y)=\left\{x, x u^{2}\right\}$ for some $x, u \in \mathbb{C}^{\times}$, we get $\operatorname{Spec}(A)=$ $\left\{x, x u^{2}, x u,-x u\right\}$. Hence age* $(A) \geq 1 / 2$ by Lemma 2.20 . Now by Lemma 2.2(iv) we have age $(g) \geq 2 \operatorname{age}^{*}(A) \geq 1$. This lower bound is best possible as age $\left(\mathbf{j} \otimes I_{2}\right)=1$ (in fact, $\mathbf{j} \otimes I_{2}$ acts as a bireflection on $V$ ).

3) We have shown that any element of $G$ that acts non-trivially on the set of three tensor factors of $V$ has age* $\geq 1$. In particular, if age* $(h)<1$ for some $h \in G$, then $h$ belongs to the base subgroup $G \cap G L\left(V_{1}\right) \otimes G L\left(V_{2}\right) \otimes G L\left(V_{3}\right)$. Thus $Z(G L(V)) \cdot\langle h \in G$ | age* $(h)<1\rangle<Z(G L(V)) G$.

\subsection{Proof of Theorem 1.7}

Let $d:=\operatorname{dim}(V)>4$ and let $G<G L(V)$ satisfy the hypotheses of the theorem. If $G$ is imprimitive, then the statement follows from Lemma 5.2 and Theorem 5.5(ii). So we may assume that the $G$-module $V$ is primitive. Now by Corollary 5.13, $V$ is tensor indecomposable, and so it cannot be tensor induced by Proposition 5.14. The extraspecial case cannot occur either, by the results of $\S 5.2$. Thus $G$ is almost quasi-simple by [GT3, Proposition 2.8], i.e. $S \triangleleft G / Z(G) \leq \operatorname{Aut}(S)$ for some simple non-abelian group $S$.

We can now apply Theorem 3.8; in particular, either $(d, S)=\left(n-1, \mathrm{~A}_{n}\right)$ or $d \leq 8$. In the former case, up to scalars, $G=\mathrm{S}_{n}$ (in its action on the deleted natural permutation module) and so a c.r.g. Consider the latter case. If $d=8$, then $S=\Omega_{8}^{+}(2), G / Z(G)=$ $S \cdot 2$, and up to scalars, $G$ is the Weyl group of type $E_{8}$. If $d=7$, then $G / Z(G)=S=$ $S p_{6}(2)$, and up to scalars, $G$ is the Weyl group of type $E_{7}$. Assume $d=6$. If $S=S U_{4}(2)$, then $G / Z(G)=S \cdot 2$, and up to scalars, $G$ is the Weyl group of type $E_{6}$. If $S=P S U_{4}(3)$, then $G / Z(G)=S \cdot 2_{2}$ in the notation of [Atlas] (the other two involutions in $\operatorname{Out}(S)$ do not preserve the 6-dimensional representation in question of $\left.G^{(\infty)}\right)$, and so $G$ is a 
c.r.g. modulo scalars. In all these cases, there is only one conjugacy class in $G / Z(G)$ that contains non-central elements $g$ with age* $(g)<1$, and these elements $g$ are scalar multiples of reflections. Finally, if $d=5$, then $G=S U_{4}(2) \cdot Z(G)$ and so it is also a c.r.g. modulo scalars.

\subsection{Proof of Theorem 1.4}

Let $(G, V)$ satisfy the hypotheses of the theorem. If the $G$-module $V$ is imprimitive, then the statement follows from Lemmas 5.2 and 5.3. (Notice that in case (iii) the transpositions in the subgroup $S_{n}$ act on $V$ as bireflections.) So we may assume that $V$ is primitive, of dimension $\geq 11$. Hence the extraspecial case cannot occur by the analysis in \$5.2. Next, the $G$-module $V$ cannot be tensor decomposable or tensor induced by Lemma 5.10 and Proposition 5.14. Thus we are in the almost quasi-simple case and can apply Theorem 3.8. Since $\operatorname{dim}(V) \geq 11$, we arrive at conclusion (i).

\subsection{Proof of Theorem 1.5}

Let $(G, V)$ satisfy the hypotheses of the theorem. First we consider the case where the $G$-module $V$ is imprimitive and apply Lemma 5.2. In the case (ii) of Lemma 5.2, we arrive at conclusion (ii) of the theorem (notice that all elements of $G$ with age* $<1$ are contained in $D$ and so cannot generate $G$ modulo scalars). Suppose we are in the case (i) of Lemma 5.2. Then $G$ satisfies the hypotheses of Theorem 5.5(i), and so we are done. So we may assume that $V$ is primitive and $\operatorname{dim}(V) \geq 9$. Hence the extraspecial case cannot occur by the analysis in $\$ 5.2$. Next, the $G$-module $V$ cannot be tensor decomposable or tensor induced by Lemma 5.11 and Proposition 5.14. Thus we are in the almost quasisimple case and can apply Theorem 3.8. Since $\operatorname{dim}(V) \geq 9$, we must now have $G=\mathrm{S}_{d+1}$ modulo scalars, as stated in (i).

Remark 5.15. (a) The group $\left(C_{5} \times S L_{2}(5)\right) *\left(C_{3} \times S p_{4}(3)\right)<G L_{8}(\mathbb{C})$ is generated by its elements of age $<1$, yet does not contain any complex reflection by Lemma 5.12 . Thus the bound $d \geq 9$ in Theorem 1.5 is best possible.

(b) The case (ii) of Theorem 1.5 indeed occurs, as shown in the following example. Consider the subgroup $A=C_{7} \times S L_{2}(5)$ of $G L_{2}(\mathbb{C})$ and let $G$ be the wreath product $A 2 S_{n}$ acting on $V=\mathbb{C}^{2 n}$ for any $n \geq 2$. It is easy to check that $G$ is generated by its (noncentral) elements with age $\leq 1$, and $G$ contains non-central elements with age $=2 / 7$. However, $G$ does not contain any complex reflection. For, suppose $g \in G$ is conjugate to $\operatorname{diag}(\alpha, \alpha, \ldots, \alpha, \beta)$ for some $\alpha \neq \beta \in S^{1}$. By Lemma 5.2, $g=\operatorname{diag}\left(a_{1}, \ldots, a_{n}\right) \in A^{n}$ since $0<\operatorname{age}^{*}(g)<1$. It follows that $\alpha^{14}=1$ and that some element $x \in S L_{2}$ (5) has eigenvalues $\mu \alpha, \mu \beta$ for some $\mu \in S^{1}$ with $\mu^{7}=1$. Now $(\mu \alpha)^{14}=1$, and so $x$ must be scalar, whence $\alpha=\beta$, a contradiction.

\subsection{Proof of Corollary 1.2}

By assumption, $2 \pi\|g\| \leq L$ for some $1 \neq g \in G$. Let $N$ be the (normal) subgroup generated by all elements in $G$ with this property. By Corollary 2.12, $N$ is generated by 
a set of elements $g$ with $d_{2}(g)^{2} \leq C$, where $C=\max \left\{4, L^{2}\right\}$. Also by [GT3, Lemma 2.5], either $N \leq Z(G)$, or $N$ is irreducible on $V$. In the former case, the cyclic group $Z(G)$ contains an element $g=e^{2 \pi i j / s} \cdot 1_{V}$ with $s:=|Z(G)|, 1 \leq j \leq s-1$, and $L \geq 2 \pi\|g\| \geq 2 \pi \sqrt{\operatorname{dim}(V)} / s$, whence $\operatorname{dim}(V) \leq(L s / 2 \pi)^{2}$. In the latter case, we may apply Theorem 1.1 to $N$. Assume that the conclusion (i) of Theorem 1.1 does not hold; in particular, $d:=\operatorname{dim}(V)>40 C \geq 160$. In case the conclusion (ii) of Theorem 1.1 holds for $N$, we have $G \triangleright M:=N^{(\infty)} \cong \mathrm{A}_{d+1}$ and $M$ acts irreducibly on $V$. By Schur's Lemma, $C_{G}(M)=Z(G)$, and $G / C_{G}(M) \leq \operatorname{Aut}(M)=M \cdot 2$, whence the conclusion (ii) of Theorem 1.1 holds for $G$.

Finally, assume that the conclusion (iii) of Theorem 1.1 holds for $N$, and let $D$ be the normal subgroup of $N$ that fixes each $V_{i}$ (setwise); in particular, $\mathrm{A}_{m} \leq N / D \leq \mathrm{S}_{m}$. In this case, $40 C<d=m \operatorname{dim}\left(V_{1}\right) \leq m C / 4$, whence $m>160$. If, in addition, $m \leq C / 4+1$, then $C \geq 640$ and so $d \leq m C / 4 \leq(C / 4+1) C / 4<4 C^{2} / 63 \leq \mathfrak{f}(C)$. Hence we may assume $m>\max \{160, C / 4+1\}$. Set $e:=\operatorname{dim}\left(V_{1}\right)$ and let $\mathcal{C}$ be the collection of all finite simple groups $S$ with the property that either $S$ is cyclic, or $S \cong X / Y$ for some finite subgroups $Y \triangleleft X<P G L_{e}(\mathbb{C})$. Observe that every composition factor of $D$ belongs to $\mathcal{C}$. (Indeed, consider the chain $D=D_{0} \triangleright D_{1} \cdots \triangleright D_{m}=1$, where $D_{i}$ is the kernel of the action of $D_{i-1}$ on $V_{i}$ for $1 \leq i \leq m$. Now let $S$ be any non-abelian composition factor of $D_{i} / D_{i-1}$. Thus $S \cong A / B$ for some $B \triangleleft A<G L\left(V_{i}\right)$ since $D_{i} / D_{i-1} \hookrightarrow G L\left(V_{i}\right)$. Since $S$ is non-abelian, $S$ is also a composition factor of $A Z / B Z$ for $Z:=Z\left(G L\left(V_{i}\right)\right)$. It follows that $S$ is a composition factor of $A Z / Z<P G L\left(V_{i}\right) \cong P G L_{e}(\mathbb{C})$, i.e. $S \in \mathcal{C}$.) Let $R$ be the largest normal subgroup of $N$ with every composition factor belonging to $\mathcal{C}$ (cf. Lemma 2.26). Then $D \triangleleft R \triangleleft N$. Assume that $R>D$. Then $\mathrm{A}_{m}$ is a composition factor of $R$ and so $\mathrm{A}_{m} \in \mathcal{C}$. The latter inclusion means that $\mathrm{A}_{m} \cong X / Y$ for some finite subgroups $Y \triangleleft X<P G L_{e}(\mathbb{C})$, with $e \leq C / 4<m-1$ and $m>160$. This however contradicts the Feit-Tits Theorem (cf. [KIL, Theorem 3]). Thus $R=D$. By Lemma 2.26, $R \triangleleft G$. We have shown that $D \triangleleft G$. Since $D$ is reducible on $V$, by [GT3, Lemma 2.5] we must have $D \leq Z(G) \cap N=Z(N)$. It follows that $V$ yields an irreducible projective representation of degree $m e \leq m C / 4<m^{2}$ of $N / D \in\left\{\mathrm{A}_{m}, \mathrm{~S}_{m}\right\}$. Recall that $m>160$. Using the information on the small degrees of irreducible projective representations of $N / D$ as given in [Ra] and [KT], we see that $m e=m(m-3) / 2, M=N^{(\infty)} \cong \mathrm{A}_{m}$, and $\left.V\right|_{M}$ equals the restriction of the Specht module $S^{(m-2,2)}$ (labeled by the partition $(m-2,2))$ to $\mathrm{A}_{m}$. But the latter restriction is primitive, whereas $V$ is imprimitive, a contradiction.

\subsection{Proof of Corollary 1.6}

By the assumption and the Reid-Tai criterion [R1], age $(g) \leq 1$ for some $1 \neq g \in G$. Let $N$ be the (normal) subgroup generated by all elements in $G$ with this property. By [GT3, Lemma 2.5], either $N \leq Z(G)$, or $N$ is irreducible on $V=\mathbb{C}^{d}$. In the former case, the cyclic group $Z(G)$ contains an element $g=e^{2 \pi i j / s} \cdot 1_{V}$ with $s:=|Z(G)|$, $1 \leq j \leq s-1$, and $1 \geq \operatorname{age}(g) \geq \operatorname{dim}(V) / s$, whence $\operatorname{dim}(V) \leq s$. In the latter case, we may apply Theorem 1.4 to $N$. Assume that the conclusion (i) of Theorem 1.1 holds for $N$. Then $G \triangleright M:=N^{(\infty)} \cong \mathrm{A}_{d+1}$ and $M$ acts irreducibly on $V$. By Schur's Lemma, 
$C_{G}(M)=Z(G)$, and $G / C_{G}(M) \leq \operatorname{Aut}(M)=M \cdot 2$, whence the conclusion (i) of

Corollary 1.6 holds for $G$.

Next, assume that either the conclusion (ii) or (iii) of Theorem 1.4 holds for $N$. In the case of (ii), define $D$ to be the normal subgroup of $N$ that fixes each $V_{i}$ (setwise); in the case of (iii), consider the normal subgroup $D$ defined therein. In particular, $\mathrm{A}_{n} \leq$ $N / D \leq \mathrm{S}_{n}$. Also, let $\mathcal{C}$ be the collection of all finite simple groups $S$ with the property that either $S$ is cyclic, or $S \cong X / Y$ for some finite subgroups $Y \triangleleft X<P G L_{2}$ (C) (and so $S \cong A_{5}$, as easily seen). Notice $n \geq 6$ since $n \geq d / 2$. Arguing as in the last part of the proof of Corollary 1.2, we see that $D$ is the largest normal subgroup of $N$ with all composition factors belonging to $\mathcal{C}$, and so $D \triangleleft G$ by Lemma 2.26. Since $D$ is reducible on $V$, by [GT3, Lemma 2.5] we must have $D \leq Z(G) \cap N=Z(N)$. Now in the case of the conclusion (iii) of Theorem 1.4 for $N$, we have $N=S_{n} Z(N)$, with $S_{n}$ acting reducibly on $V$, a contradiction. Thus we are in the case of the conclusion (ii) of Theorem 1.4, in particular $n \geq 11$, and $V$ yields an irreducible projective representation of degree $n$ of $N / D \in\left\{\mathrm{A}_{n}, \mathrm{~S}_{n}\right\}$, again a contradiction.

\subsection{Small dimension case}

Proposition 5.16. Let $G$ satisfy the set-up $(\mathbf{A})$, with $4 \leq d:=\operatorname{dim}(V) \leq 10$. Then one of the following statements holds.

(i) G preserves a decomposition $V=V_{1} \oplus \cdots \oplus V_{d}$ of $V$ into 1-spaces, and $(\pi(G), d)=$ $\left(\mathrm{S}_{d}, d\right),\left(\mathrm{A}_{d}, d\right),\left(A S L_{3}(2), 8\right),\left(S L_{3}(2), 7\right),\left(\mathrm{A}_{5}, 6\right),\left(D_{10}, 5\right)$, if $\pi$ denotes the induced permutation action of $G$ on $\left\{V_{1}, \ldots, V_{d}\right\}$.

(ii) $2 \mid d$, and $G=D: \mathrm{S}_{d / 2}<G L_{2}(\mathbb{C}) ? \mathrm{~S}_{d / 2}$, a split extension of $D<G L_{2}(\mathbb{C})^{d / 2}$ by $\mathrm{S}_{d / 2}$.

(iii) $G$ preserves a decomposition $V=A \otimes B$, with $\operatorname{dim}(A), \operatorname{dim}(B)>1$.

(iv) $G$ preserves a tensor structure $V=A^{\otimes m}$, with $\operatorname{dim}(A)=2$ and $m=2,3$.

(v) $4 \leq \operatorname{dim}(V)=p^{a} \leq 9$ for some prime $p$, and $G$ normalizes a $p$-group $E$ of extraspecial type, with $|E / Z(E)|=p^{2 a}$.

(vi) $G$ is almost quasi-simple, and $G$ satisfies the conclusions of Theorem 3.8.

Proof. If the $G$-module $V$ is imprimitive, then the statement follows from Lemma 5.2. Assume $V$ is primitive. Next, (iii), resp. (iv), (v), corresponds to the case when the $G$ module $V$ is tensor decomposable, resp. tensor induced, or $G$ is in the extraspecial case. Otherwise, by [GT3, Proposition 2.8] $G$ satisfies the hypothesis, and so the conclusions, of Theorem 3.8.

Acknowledgments. Part of this paper was written while the authors were participating in the Representations of Finite Groups Program of the Mathematical Sciences Research Institute (Berkeley, 2008), and the Algebraic Lie Theory Program of the Isaac Newton Institute for Mathematical Sciences (Cambridge, 2009). It is a pleasure to thank the MSRI and the Newton Institute for their generous hospitality and support.

The authors would like to thank János Kollár for suggesting this problem to them, and for many insightful comments on the paper. They also thank Thomas Breuer for computing the character tables of certain groups of extraspecial type for them, and Lennie Friedlander, Jason Fulman, Nick Katz, Klaus Lux, and Terence Tao for helpful remarks on various aspects of the paper. 
The authors gratefully acknowledge the support of the NSF (grants DMS-0653873 and DMS0901241).

\section{References}

[A] Aschbacher, M.: On the maximal subgroups of the finite classical groups. Invent. Math. 76, 469-514 (1984) Zbl 0537.20023 MR 0746539

[Be] Benson, D.: Polynomial Invariants of Finite Groups. London Math. Soc. Lecture Note Ser. 190, Cambridge Univ. Press, Cambridge (1993) Zbl 0864.13001 MR 1249931

[BH] Beukers, F., Heckman, G.: Monodromy for the hypergeometric function ${ }_{n} F_{n-1}$. Invent. Math. 95, 325-354 (1989) Zbl 0663.30044 MR 0974906

[B] Bourbaki, N.: Groupes et Algèbres de Lie. Ch. IV-VI, Hermann, Paris (1968) Zbl 0186.33001 MR 0240238

[BG] Bryan, J., Graber, T.: The crepant resolution conjecture. In: Algebraic Geometry-Seattle 2005, Part 1, Proc. Sympos. Pure Math. 80, Part 1, Amer. Math. Soc., Providence, RI, $23-$ 42 (2009) Zbl 1198.14053 MR 2483931

[CR] Chen, W., Ruan, Y.: A new cohomology theory of orbifold. Comm. Math. Phys. 248, 1-31 (2004) Zbl 1063.53091 MR 2104605

[Co] Cohen, A.: Finite quaternionic reflection groups. J. Algebra 64, 293-324 (1980) Zbl 0433.20035 MR 0579063

[Atlas] Conway, J. H., Curtis, R. T., Norton, S. P., Parker, R. A., Wilson, R. A.: An ATLAS of Finite Groups. Clarendon Press, Oxford (1985) Zbl 0568.20001 MR 0827219

[CK] Cox, D. A., Katz, S.: Mirror Symmetry and Algebraic Geometry. Math. Surveys Monogr. 68, Amer. Math. Soc., Providence, RI (1999) Zbl 0951.14026 MR 1677117

[D] Dornhoff, L.: Group Representation Theory. Dekker, New York (1972)

[FH] Fulton, W., Harris, J.: Representation Theory. Springer, New York (1991) Zbl 0744.22001 MR 1153249

[GAP] The GAP group: GAP—Groups, Algorithms, and Programming. Version 4.4 (2004); http://www.gap-system.org

[G] Gluck, D.: Sharper character value estimates for groups of Lie type. J. Algebra 174, 229_ 266 (1995) Zbl 0842.2001 MR 1332870

[GI] Gluck, D., Isaacs, I. M.: Tensor induction of generalized characters and permutation characters. Illinois J. Math. 27, 514-518 (1983) Zbl 0512.20006 MR 0698312

[GM] Gluck, D., Magaard, K.: Base sizes and regular orbits for coprime affine permutation groups. J. London Math. Soc. 58, 603-618 (1998) Zbl 0951.20003 MR 1678153

[Go1] Gordeev, N. L.: Invariants of linear groups generated by matrices with two eigenvalues different from one. Zap. Nauchn. Sem. Leningrad. Otdel. Mat. Inst. Steklov. (LOMI) 114, 120-130, 219 (1982) (in Russian) Zbl 0499.20030 MR 0669563

[Go2] Gordeev, N. L.: Finite linear groups whose algebras of invariants are complete intersections. Math. USSR-Izv. 28, 335-379 (1987) Zbl 0626.14009

[GMST] Guralnick, R. M., Magaard, K., Saxl, J., Pham Huu Tiep: Cross characteristic representations of symplectic groups and unitary groups. J. Algebra 257, 291-347 (2002) Zbl 1025.20002 MR 1947325

[GS] Guralnick, R. M., Saxl, J.: Generation of finite almost simple groups by conjugates. J. Algebra 268, 519-571 (2003) Zbl 1037.20016 MR 2009321

[GT1] Guralnick, R. M., Pham Huu Tiep: Cross characteristic representations of even characteristic symplectic groups. Trans. Amer. Math. Soc. 356, 4969-5023 (2004) Zbl 1062.20013 MR 2084408 
[GT2] Guralnick, R. M., Pham Huu Tiep: The non-coprime $k(G V)$-problem. J. Algebra 293, 185-242 (2005) Zbl 1083.20006 MR 2173972

[GT3] Guralnick, R. M., Pham Huu Tiep: Symmetric powers and a problem of Kollár and Larsen. Invent. Math. 174, 505-554 (2008) Zbl pre05530511 MR 2453600

[Ha] Haiman, M.: Vanishing theorems and character formulas for the Hilbert scheme of points in the plane. Invent. Math. 149, 371-407 (2002) Zbl 1053.14005 MR 1918676

[Hep] Hepworth, R.: The age grading and the Chen-Ruan cup product. Bull. London Math. Soc. 42, 868-878 (2010) Zbl 1205.55006 MR 2721746

$[\mathrm{H}] \quad$ Hiss, G.: Zerlegungszahlen endlicher Gruppen vom Lie-Typ in nicht-definierender Charakteristik. Habilitationsschrift, RWTH Aachen (1990)

$[\mathrm{Hu}]$ Huffman, W. C.: Imprimitive linear groups generated by elements containing an eigenspace of codimension two. J. Algebra 63, 499-513 (1980) Zbl 0435.20030 MR 0570727

[HW] Huffman, W. C., Wales, D. B.: Linear groups containing an involution with two eigenvalues -1. J. Algebra 45, 465-515 (1977) Zbl 0364.20046 MR 0435243

[IR] Ito, Y., Reid, M.: The McKay correspondence for finite subgroups of SL(3, C). In: Higher-Dimensional Complex Varieties (Trento, 1994), de Gruyter, Berlin, 221-240 (1996) Zbl 0894.14024 MR 1463181

[KW] Kac, V., Watanabe, K.: Finite linear groups whose ring of invariants is a complete intersection. Bull. Amer. Math. Soc. 6, 221-223 (1982) Zbl 0483.14002 MR 0640951

[Ka] Kaledin, D.: On crepant resolutions of symplectic quotient singularities. Selecta Math. 9, 529-555 (2003) Zbl 1066.14003 MR 2031751

[KM] Kemper, G., Malle, G.: The finite irreducible linear groups with polynomial ring of invariants. Transform. Groups 2, 57-89 (1997) Z Zbl 0899.13004 MR 1439246

[KIL] Kleidman, P. B., Liebeck, M. W.: On a theorem of Feit and Tits. Proc. Amer. Math. Soc. 107, 315-322 (1989) Zbl 0688.20005 MR 0961412

[KP] Kleshchev, A. S., Premet, A. A.: On second degree cohomology of symmetric and alternating groups. Comm. Algebra 21, 583-600 (1993) Zbl 0798.20046 MR 1199691

[KT] Kleshchev, A. S., Pham Huu Tiep: On restrictions of modular spin representations of symmetric and alternating groups. Trans. Amer. Math. Soc. 356, 1971-1999 (2004) Zbl 1065.20013 MR 2031049

[KL] Kollár, J., Larsen, M.: Quotients of Calabi-Yau varieties. In: Algebra, Arithmetic, and Geometry: In Honor of Yu. I. Manin, Vol. II, Progr. Math. 270, Birkhäuser, Boston, MA, 179-211 (2009) Zbl 1200.14074 MR 2641190

[LS] Liebeck, M., Saxl, J.: Minimal degrees of primitive permutation groups, with an application to monodromy groups of covers of Riemann surfaces. Proc. London Math. Soc. 63, 266-314 (1991) Zbl 0696.20004 MR 1114511

[Lu1] Lübeck, F.: Smallest degrees of representations of exceptional groups of Lie type. Comm. Algebra 29, 2147-2169 (2001) Zbl 1004.20003 MR 1837968

[Lu2] Lübeck, F.: Character degrees and their multiplicities for some groups of Lie type of rank $<9$. http://www.math.rwth-aachen.de/ Frank.Luebeck/chev/DegMult/index.html

[MOW] Metcalfe, A. P., O'Connell, N., Warren, J.: Interlaced processes on the circle. Ann. Inst. H. Poincaré Probab. Statist. 45, 1165-1184 (2009)

[N] Nakajima, H.: Quotient singularities which are complete intersections. Manuscripta Math. 48, 163-187 (1984) Zbl 0577.14038 MR 0753729

[Ra] Rasala, R.: On the minimal degrees of characters of $S_{n}$. J. Algebra 45, 132-181 (1977) Zbl 0348.20009 MR 0427445 
[R1] Reid, M.: Canonical 3-folds. In: Algebraic Geometry, Angers, 1979, Sijthoff \& Noordhoff, Alphen aan den Rijn and Germantown, MD, 273-310 (1980) Z Zbl 0451.14014 MR 0605348

[R2] Reid, M.: La correspondance de McKay. In: Séminaire Bourbaki, Vol. 1999/2000, Astérisque 276, 53-72 (2002) Zbl 0996.14006 MR 1886756

[Ru] Ruan, Y.: The cohomology ring of crepant resolutions of orbifolds. In: Gromov-Witten Theory of Spin Curves and Orbifolds, Contemp. Math. 403, Amer. Math. Soc., Providence, RI, 117-126 (2006) Zbl 1105.14078 MR 2234886

[S] Serre, J.-P.: Groupes finis d'automorphismes d'anneaux locaux réguliers. In: Colloque d'Algèbre (Paris, 1967), exp. 8, 11 pp. Zbl 0200.00002 MR 0234953

[ST] Shephard, G. C., Todd, J. A.: Finite unitary reflection groups. Canad. J. Math. 6, 274-304 (1954) Zbl 0055.14305 MR 0059914

[Sm] Smith, L.: Polynomial Invariants of Finite Groups. A. K. Peters, Wellesley, MA (1995) Zbl 0864.13002 MR 1328644

[Sp] Spaltenstein, N.: Charactères unipotents de ${ }^{3} D_{4}\left(\mathbb{F}_{q}\right)$, Comment. Math. Helv. 57, 676691 (1982) Zbl 0536.20025 MR 0694610

[Sr] Srinivasan, B.: The characters of the finite symplectic group $S p(4, q)$. Trans. Amer. Math. Soc. 131, 488-525 (1968) Zbl 0213.30401 MR 0220845

[St] Steinberg, R.: The representations of $G L(3, q), G L(4, q), P G L(3, q)$, and $P G L(4, q)$. Canad. J. Math. 3, 225-235 (1951) Zbl 0042.25602 MR 0041851

[Tao] Tao, T.: private communication

[T] Pham Huu Tiep: Finite groups admitting grassmannian 4-designs. J. Algebra 306, 227243 (2006) Zbl 1113.51005 MR 2271581

[TZ1] Pham Huu Tiep, Zalesskii, A. E.: Minimal characters of the finite classical groups, Comm. Algebra 24, 2093-2167 (1996) Zbl 0901.20031 MR 1386030

[TZ2] Pham Huu Tiep, Zalesskii, A. E.: Some characterizations of the Weil representations of the symplectic and unitary groups. J. Algebra 192, 130-165 (1997) Zbl 0877.20030 MR 1449955

[V] Verbitsky, M.: Holomorphic symplectic geometry and orbifold singularities. Asian J. Math. 4, 553-563 (2000) Zbl 1018.32028 MR 1796694

[Wa] Wales, D. B.: Linear groups of degree $n$ containing an involution with two eigenvalues -1. II. J. Algebra 53, 58-67 (1978) Zbl 0404.20034 MR 0480770

[W] Wielandt, H.: Finite Permutation Groups. Academic Press, New York (1964) Zbl 0138.02501 MR 0183775 